\title{
CAUSAL GEOMETRY OF EINSTEIN-VACUUM SPACETIMES WITH FINITE CURVATURE FLUX
}

\author{
SERGIU KLAINERMAN AND IGOR RODNIANSKI
}

\begin{abstract}
One of the central difficulties of settling the $L^{2}$-bounded curvature conjecture for the Einstein -Vacuum equations is to be able to control the causal structure of spacetimes with such limited regularity. In this paper we show how to circumvent this difficulty by showing that the geometry of null hypersurfaces of Enstein-Vacuum spacetimes can be controlled in terms of initial data and the total curvature flux through the hypersurface.
\end{abstract}

\section{INTRODUCTION}

We consider the Einstein Vacuum equations,

$$
\mathbf{R}_{\alpha \beta}=0
$$

where $\mathbf{R}_{\alpha \beta}$ denotes the Ricci curvature tensor of a four dimensional Lorentzian space time $(\mathcal{M}, \mathbf{g})$. The fundamental problem of the subject is to study the long term regularity and asymptotic properties of the Cauchy developments of general, asymptotically flat, initial data $\operatorname{sets}^{1}(\Sigma, g, k)$. In so far as local regularity is concerned it is natural to ask what are the minimal local regularity properties of the initial data which guarantee the existence and uniqueness of local developments.

The first step in this direction was taken by Y. C. Bruhat $[\mathrm{Br}]$ who proved a local in time existence result under the assumption ${ }^{2}$ that $g \in H_{\mathrm{loc}}^{s}(\Sigma), k \in H_{\mathrm{loc}}^{s-1}(\Sigma)$ with $k \geq 4$. The result, which was was later improved to $s>5 / 2$ in [H-K-M], depended only on energy estimates and Sobolev type inequalities and did not require the study of null hypersurfaces. It was conjectured ${ }^{3}$ in [Kl1] that one can significantly improve the result to $s=2$ which corresponds to initial data sets with bounded $L^{2}$ curvature, result which would be particularly satisfying in view of the naturalness

1991 Mathematics Subject Classification. 35J10

The first author is partially supported by NSF grant DMS-0070696. The second author is a Clay Prize Fellow and is partially supported by NSF grant DMS-01007791 .

${ }^{1}$ Though the results presented here are local in nature and apply equally well to more general data.

${ }^{2}$ These are local Sobolev spaces on $\Sigma$; more precise conditions can be given in terms of weighted Sobolev spaces at infinity.

${ }^{3}$ In [Kl1] the issue was also raised of going all the way to the critical exponent $s_{c}=3 / 2$. We shall argue below that this may not be possible, see also footnote 7 below. 
of the norms involved. The conjecture was motivated by the progress made earlier on semilinear type equations such as Wave Maps and Yang Mills equations.

At that time the conjecture was made it seemed however completely out of reach. It was clear that in order to improve the exponent $s>5 / 2$ one had to abandon the naive use of Sobolev inequalities of the classical argument and rely instead on Strichartz and bilinear type estimates. The problem was that one needed to extend these estimates to wave operators on very rough background metrics. The first results below 5/2 are due to Bahouri-Chemin [Ba-Ch1], Tataru [Ta], [Kl-Rodn1] and relied, indeed, on proving Strichartz type estimates on such backgrounds. To do this they had to rely on adequate notions of approximate fundamental solutions( or vectorfields in the case of [Kl-Rodn1]) for the corresponding wave operators based on an adapted version of the classical geometric optics construction. This construction depends heavily on the regularity properties of null hypersurfaces associated to these backgrounds and applies to general type of quasilinear wave equations including the reduced Einstein vacuum equations in wave coordinates. In [Kl-Rodn2] we were able to reach, for the particular case of Einstein vacuum equations, any exponent $s>2$. A similar result was also obtained in [Sm-Ta] for the general class of quasilinear wave equations mentioned above.

The case $s=2$ is far more difficult. First of all such a result cannot hold for general quasilinear wave equations, see [Lind]. As the experience with semilinear wave equations demonstrates, see discussion in [Kl1], to prove such a result we need the following ingredients:

(1) Provide a system of coordinates relative to which (1) verifies an appropriate version of the null condition.

(2) Prove appropriate bilinear estimates for solutions to homogeneous wave equations, of the type $\square_{\mathbf{g}} \phi=0$, on a fixed Einstein Vacuum background( endowed with the coordinate system indicated in 1.) with bounded $L^{2}$ curvature.

To prove bilinear estimates we need a good notion of approximate solutions; this requires the third ingredient, typical to quasilinear equations,

3. Make sense of null hypersurfaces, on Einstein vacuum backgrounds with only $L^{2}$ bounds on their curvature tensor, and provide appropriate estimates on their geometry.

In this paper we make a first step towards settling the bounded $L^{2}-$ curvature conjecture by providing the main ideas and techniques needed to deal with the last ingredient mentioned above. More precisely we shall consider an outgoing null hypersurface ${ }^{4} \mathcal{H}$, initiating on a compact 2 surface $S_{0} \subset \Sigma$ diffeomorphic to $\mathbf{S}^{\mathbf{2}}$,

\footnotetext{
${ }^{4}$ In this paper, for clarity, we only consider spherical geodesic foliations of a fixed null hypersurface. Nevertheless our methods can be easily extended to deal with other foliations and other type of null hypersurfaces. They can also be extended to families of null hypersurfaces as it would be in fact needed to tackle the $L^{2}$ curvature conjecture.
} 
given by the level hypersurfaces of an optical function $u$, i.e. solution to the Eikonal equation

$$
\mathbf{g}^{\alpha \beta} \partial_{\alpha} u \partial_{\beta} u=0
$$

Let $L=-\mathbf{g}^{\alpha \beta} \partial_{a} u \partial_{b}$ be the corresponding null generator vectorfield and $s$ its affine parameter, i.e. $L(s)=1,\left.\quad s\right|_{S_{0}}=0$. The level surfaces $S_{s}$ of $s$ generates the geodesic foliation on $\mathcal{H}$. We shall denote by $\nabla$ the covariant differentiation on $S_{s}$ and by $\nabla_{L}$ the projection to $S_{s}$ of the covariant derivative with respect to $L$, see section 2. We also denote by $r$ the function on $\mathcal{H}$ defined by $r=r(s)=$ $\sqrt{(4 \pi)^{-1}\left|S_{s}\right|}$, with $\left|S_{s}\right|$ the area of $S_{s}$. Let $\mathcal{H}_{t}$ be the portion of $\mathcal{H}$ between $s=0$ and $s=t$ and, for simplicity, assume $\mathcal{H}=\mathcal{H}_{1}$. We introduce the total curvature flux ${ }^{5}$ along $\mathcal{H}$ to be the integral, see precise definition in section 2 ,

$$
\mathcal{R}_{0}=\left(\|\alpha\|_{L^{2}(\mathcal{H})}^{2}+\|\beta\|_{L^{2}(\mathcal{H})}^{2}+\|\rho\|_{L^{2}(\mathcal{H})}^{2}+\|\sigma\|_{L^{2}(\mathcal{H})}^{2}+\|\underline{\beta}\|_{L^{2}(\mathcal{H})}^{2}\right)^{\frac{1}{2}}
$$

with $\alpha, \beta, \rho, \sigma, \beta$ null components of the curvature tensor $\mathbf{R}$. More precisely, for any vectorfields $X, Y$ on $\mathcal{H}$ tangent to the $s$ foliation,

$$
\alpha(X, Y)=\mathbf{R}(X, L, Y, L), \quad \beta(X)=\frac{1}{2} \mathbf{R}(X, L, \underline{L}, L), \quad \rho=\frac{1}{4} \mathbf{R}(L, \underline{L}, L, \underline{L}) \ldots
$$

see definition 2.4 for the other components $\sigma, \beta$. We shall assume that the total curvature flux $\mathcal{R}_{0}$ is finite and show that the geometry of the null hypersurface $\mathcal{H}_{t}$, for sufficiently small $t>0$, depends only on initial conditions on $S_{0}$, which we denote by $\mathcal{I}_{0}$ and will make precise later, and the size of $\mathcal{R}_{0}$. Alternatively we will take $t=1$ and make $\mathcal{R}_{0}$ and $\mathcal{I}_{0}$ sufficiently small ${ }^{6}$.

The geometry of $\mathcal{H}$ depends in particular of the null second fundamental form

$$
\chi(X, Y)=<D_{X} L, Y>
$$

with $X, Y$ arbitrary vectorfields tangent to the $s$-foliation. We denote by $\operatorname{tr} \chi$ the trace of $\chi$, i.e. $\operatorname{tr} \chi=\delta^{a b} \chi_{a b}$ where $\chi_{a b}$ are the components of $\chi$ relative to an orthonormal frame $\left(e_{a}\right)_{a=1,2}$ on the leaves of the $s$-foliation. It satisfies the well known Raychadhouri equation, see (60),

$$
\frac{d}{d s} \operatorname{tr} \chi+\frac{1}{2}(\operatorname{tr} \chi)^{2}=-|\hat{\chi}|^{2}
$$

with $\hat{\chi}_{a b}=\chi_{a b}-\frac{1}{2} \operatorname{tr} \chi \delta_{a b}$ the traceless part of $\chi$.

Even in Minkowski space one cannot adequately control the geometry of null hypersurfaces without a uniform bound on, at least, $\operatorname{tr} \chi$. Indeed one can show by an explicit calculation, see section 2.36, that if $L^{\infty}$ norm of $\operatorname{tr} \chi$, on an initial 2-surface, is allowed to become arbitrarily large caustics can occur instantaneously and therefore all $L^{p}$ norms, $p>2$ must become infinite ${ }^{7}$. To avoid caustics we are thus led to ask whether we can bound the $L^{\infty}$ norm of $\operatorname{tr} \chi$ simply in terms of the initial data

\footnotetext{
${ }^{5}$ The justification for this quantity can be found in [Chr-Kl] or [Kl-Nic] in connection to the Bell Robinson tensor and the Bianchi identities verified by the curvature tensor $\mathbf{R}$.

${ }^{6}$ The smallness of $\mathcal{I}_{0}$ implies that the metric on $S_{0}$ is close to that of the standard sphere.

${ }^{7}$ This fact leads us to conjecture that one cannot control solutions of the Einstein equations with initial data rougher than $H^{2}$, i.e. in some $H^{s}, s<2$. Thus the $H^{2}$ conjecture appears to be a natural endpoint result !
} 
norm $\mathcal{I}_{0}$ and total curvature flux $\mathcal{R}_{0}$. At first glance this seems impossible. Indeed, by integrating (6), in order to control $\|\operatorname{tr} \chi\|_{L^{\infty}}$ we need to control uniformly the integrals $\int_{\Gamma}|\hat{\chi}|^{2}$, along all null geodesic generators $\Gamma$ of $\mathcal{H}$. On the other hand $\hat{\chi}$ verifies a transport equation of the form,

$$
\frac{d}{d s} \hat{\chi}+\frac{1}{2} \operatorname{tr} \chi \cdot \hat{\chi}=-\alpha
$$

where $\alpha$, the null curvature component defined by (4), is only $L^{2}$ integrable on $\mathcal{H}$. Thus, unless there is a miraculous gain of a spatial derivative(along the surfaces of the $s$ - foliation) of the integrals of $\alpha$ along the null geodesic generators $\Gamma$, we have no chance to control the $L^{\infty}$ norm of tr $\chi$. Fortunately this cancellation occurs and it is best seen by observing that $\hat{\chi}$ verifies a Codazzi type equation of the form,

$$
\operatorname{div} \hat{\chi}=-\beta+\frac{1}{2} \nabla \operatorname{tr} \chi+\frac{1}{2} \operatorname{tr} \chi \cdot \zeta-\zeta \cdot \hat{\chi}
$$

with $\beta$ as defined above and where $\zeta$ denotes the torsion of the $s$ foliation, see (17). As it was noted and made use of in [Chr-Kl], div $\hat{\chi}$ defines an elliptic system on the leaves of the $s$ foliation and therefore we expect ${ }^{8}$ that $\hat{\chi}$ behaves like $\mathcal{D}^{-1}(-\beta+$ $\left.\frac{1}{2} \nabla \operatorname{tr} \chi\right)$ with $\mathcal{D}^{-1}$ a pseudodifferential operator of order -1 . We are thus led to control, uniformly, the integrals

$$
\int_{\Gamma}\left|\mathcal{D}^{-1}\left(-\beta+\frac{1}{2} \nabla \operatorname{tr} \chi\right)\right|^{2}
$$

along the null generators $\Gamma$ or, as there is no chance of an additional cancellation between the two terms, the integrals

$$
I_{1}=\int_{\Gamma}\left|\mathcal{D}^{-1} \beta\right|^{2}, \quad I_{2}=\int_{\Gamma}\left|\mathcal{D}^{-1} \cdot \nabla \operatorname{tr} \chi\right|^{2} .
$$

Consider first $I_{1}$. Can we estimate it in terms of $\|\beta\|_{L^{2}(\mathcal{H})}$ ?. In our previous work $^{9}$ [Kl-Rodn2] we had interpreted $\beta$ as corresponding to the term $\partial^{2} \mathbf{g}+(\partial \mathbf{g})^{2}$, where $\mathbf{g}$ is a space-time metric. In wave coordinates the Einstein metric $\mathbf{g}$ solves a nonlinear wave equation which can be written schematically in the form,

$$
\mathbf{g}^{\mu \nu} \partial_{\mu \nu}^{2} \mathbf{g}=(\partial \mathbf{g})^{2} .
$$

Thus,

$$
\int_{\Gamma}\left|\mathcal{D}^{-1} \beta\right|^{2} \approx \int_{\Gamma}|\partial \phi|^{2}
$$

where, in a first approximation, we can think of $\phi$ as an $H^{2}$ solution of a linear wave equation in Minkwoski space, $\square \phi=0$. Unfortunately, it is known (see [Kl-Ma]), that the integral $\int_{\Gamma}|\partial \phi|^{2}$ can become arbitrary large, independent of length of the null segment $\Gamma$ and the size of data in $H^{2}$. This phenomenon, in fact, was used as a counterexample to the Strichartz estimate

$$
\|\partial \phi\|_{L_{t}^{2} L_{x}^{\infty}} \lesssim\left\|\phi_{0}\right\|_{H^{s}}
$$

for $s=2$. The Strichartz estimate (10), however, holds true for $s>2$. This suggests that an adapted version of it, for rough backgrounds, could be used to control the local geometry of null hypersurfaces, provided that the regularity assumptions are consistent with $\mathbf{g} \in H^{s}, s>2$. This strategy was, in fact, implemented in [Kl-Rodn2] and [Sm-Ta].

\footnotetext{
${ }^{8}$ Assuming that the remaining quadratic terms in (8) can also be controlled.

${ }^{9}$ Similar arguments were also used in [Sm-Ta].
} 
Another way of looking at the question of whether $\int_{\Gamma}\left|\mathcal{D}^{-1} \beta\right|^{2}$ can be bounded by $\|\beta\|_{L^{2}(\mathcal{H})}$ is to interpret it as a restriction problem. This makes sense dimensionally and it corresponds to a trace type theorem of the type

$$
\|U\|_{L^{2}(\Gamma)} \lesssim\|\nabla U\|_{L^{2}(\mathcal{H})} \text {. }
$$

applied to the tensor $U=\mathcal{D}^{-1} \beta$. Unfortunately it is well known that this type of trace theorems, just as the sharp Sobolev embedding into $L^{\infty}$, fail to be true. We might be able to overcome this difficulty if we could write $\beta=\nabla_{L} Q$ with $Q$ a tensor verifying ${ }^{10}\left\|\bar{\nabla}^{2} Q\right\|_{L^{2}(\mathcal{H})}+\|Q\|_{L^{2}(\mathcal{H})} \lesssim \mathcal{R}_{0}$. Indeed such a result holds true in flat space ${ }^{11}$. The breakthrough, which allows us to make use of an appropriately adapted version of the sharp trace theorem mentioned above, is provided by the Bianchi identities,

$$
D_{[\tilde{\mu}} \mathbf{R}_{\alpha \beta] \gamma \delta}=0
$$

which, expressed relative to our null pair $L, \underline{L}$, takes the form of a system of first order equations connecting the $\nabla_{L}$ derivatives of the null components $\alpha, \beta, \rho, \sigma \ldots$ to their spatial derivatives $\nabla$. In particular we have, ignoring the quadratic and higher order terms ${ }^{12}$, see (41),(42) for precise formulas,

$$
\operatorname{div} \beta=L(\rho)+\ldots, \quad \operatorname{curl} \beta=-L(\sigma)+\ldots
$$

Once more this is an elliptic Hodge system in $\beta$ and we can write, formally

$$
\mathcal{D}^{-1} \beta=\mathcal{D}^{-2} L(\rho,-\sigma)+\ldots=\nabla_{L}\left(\mathcal{D}^{-2}(\rho,-\sigma)\right)+\left[\mathcal{D}^{-2}, \nabla_{L}\right](\rho,-\sigma)+\ldots
$$

with $\mathcal{D}^{-2}$ a pseudodifferential operator of order -2 . Thus, ignoring for the moment the commutator $\left[\mathcal{D}^{-2}, \nabla_{L}\right](\rho,-\sigma)$ and the other error terms, we have indeed

$$
\mathcal{D}^{-1} \beta=\nabla_{L} Q+\ldots, \quad Q=\mathcal{D}^{-2}(\rho,-\sigma)
$$

and one can check, with the help of the null Bianchi identities and $L^{2}$ elliptic estimates, ${ }^{13}$ that $\left\|\bar{\nabla}^{2} Q\right\|_{L^{2}(\mathcal{H})}+\|Q\|_{L^{2}(\mathcal{H})}$ can indeed be bounded by $\mathcal{R}_{0}$.

This circle of ideas seem to take care of the integral $I_{1}$ in (9). Unfortunately we hit another serious obstacle with $I_{2}$. The problem is that the operator $\mathcal{D}^{-1} \cdot \nabla$ is a nonlocal operator of order zero and therefore does not map $L^{\infty}$ into $L^{\infty}$. To overcome this difficulty we are forced to try to prove a stronger estimate for $\operatorname{tr} \chi$. The idea is to try to prove the boundedness of $\operatorname{tr} \chi$ not only in $L^{\infty}$ but rather in a Besov space of type $B_{2,1}^{1}\left(S_{s}\right)$ which both imbeds in $L^{\infty}\left(S_{s}\right)$ and is stable relative to operators of order zero. This simple, unavoidable fact, forces us to work with spaces defined by Littlewood-Paley projections and therefore adds a lot of technical baggage to this work. Moreover the standard LP- theory, based on Fourier transform, would require the use of local coordinates on $\mathcal{H}$. The most natural coordinates would be those transported by the hamiltonian flow generated by $L$. Yet these transported coordinates lose $1 / 2$ derivative, relative to what one

\footnotetext{
${ }^{10}$ We denote by $\bar{\nabla}=\left(\nabla, \nabla_{L}\right)$ all first derivatives on $\mathcal{H}$ and by $\bar{\nabla}^{2} Q$ all second derivatives.

${ }^{11}$ That is the estimate $\left(\int_{\Gamma}\left|\nabla_{L} Q\right|^{2}\right)^{\frac{1}{2}} \lesssim\left\|\bar{\nabla}^{2} Q\right\|_{L^{2}(\mathcal{H})}+\|Q\|_{L^{2}(\mathcal{H})}$ holds true in flat space.

${ }^{12}$ It turns out that the ignored terms are not so easy to treat, we shall need to make various renormalizations. In particular we shall need to renormalize the Bianchi identities (13) by introducing new quantities $(\check{\rho}, \check{\sigma})$, see $(44)$.

${ }^{13}$ Ignoring the multitude of error terms generated in the process. In reality the error terms require delicate arguments.
} 
expects in view of the finite flux assumption( see proposition 4.4), and thus we prefer to rely on an invariant, geometric, version of the LP-theory. We develop such a theory, together with an appropriate paradifferential calculus, in [Kl-Rodn3] by following the heat flow approach of [Stein]. An informal introduction to the sharp trace type theorems in Besov spaces needed in our work and the geometric LP theory on which they rely is given in [Kl-Rodn4].

Yet another difficulty is due to the presence of the torsion element $\zeta$ in the Codazzi equation (8). It turns out that the estimates for $\zeta$ are at least as complex as those for $\chi$. Moreover they depend on another parameter of the foliation, the extrinsic second fundamental form $\chi$ for which we also need to provide delicate estimates. We shall provide a more complete informal description of the main circle of ideas of how to treat $\chi, \zeta, \underline{\chi}$ in section 4 after we make a full discussion of the null structure and Bianchi identities.

In addition to the difficulties already mentioned we have to confront another unexpected hurdle; to arrive at the crucial formula (14) one needs to estimate the commutators between the $\nabla_{L}$ derivative and the pseudodifferential operator $\mathcal{D}^{-2}$ applied to the curvature components $(\rho,-\sigma)$. It turns out that far from being lower order these commutators have the same level of differentiability as the principal terms and require, themselves, a nontrivial renormalization ${ }^{14}$. This difficulty, typical to end point results, is also a major technical complication in the proof of the sharp trace type results on which this work relies.

We are now ready to state our main result in a preliminary simplified version. A full version of the theorem can be found in section 3 .

Main Theorem. (First version) Consider an outgoing null hypersurface $\mathcal{H}=\mathcal{H}_{1}$, initiating on a compact 2 surface $S_{0} \subset \Sigma$ diffeomorphic to $\mathbf{S}^{2}$ and foliated by the geodesic foliation associated to the affine parameter $s$ with $\left.s\right|_{S_{0}}=0$. Assume that both the initial data quantity $\mathcal{I}_{0}$ and the total curvature flux $\mathcal{R}_{0}$ are sufficiently small. Then ${ }^{15}$,

$$
\left\|\operatorname{tr} \chi-\frac{2}{r}\right\|_{L^{\infty}(\mathcal{H})} \lesssim \mathcal{I}_{0}+\mathcal{R}_{0}
$$

Additional estimates hold true for $\hat{\chi}, \zeta$ and $\underline{\chi}$.

The proof of the theorem requires not only the material of this paper but also two other separate papers. In [Kl-Rodn3] we discuss a geometric version of LittlewoodPaley theory needed in our proof. In [Kl-Rodn4] we use this geometric LP theory to prove the trace type theorems to which we have alluded above as well as other technical results. In this paper we rely on the results of [Kl-Rodn3]-[Kl-Rodn4] to prove our main theorem.

In section 2 we discuss the main geometric quantities associated to null hypersurfaces $\mathcal{H}$, discuss the null structure and null Bianchi identities as well as various

\footnotetext{
${ }^{14} \mathrm{We}$ deal with them by an infinite sequence of renormalizations, see lemma 6.13 .

${ }^{15}$ Observe that $\frac{2}{r}$ is the value of $\operatorname{tr} \chi$ for an outgoing null cone in Minkowski space.
} 
renormalizations needed later. We refer the reader to the relevant material in [Chr-Kl] and [Kl-Nic].

In section 3 we formulate a precise version of the main theorem and give a more elaborate description of the main ideas in its proof. We also formulate the simplest bootstrap assumption BA1 and discuss some important simple consequences of it.

In section 4 we introduce our other bootstrap assumptions BA2-BA4 and discuss some of their basic consequences. We prove that the surfaces of our foliation satisfy the WS condition which allows us to use the results of [Kl-Rodn3]. We also derive estimates for the Gauss curvature $K$. We show that the $L^{2}$ norm of $K$ on the null hypersurface $\mathcal{H}$ is bounded by the total curvature flux. We also give uniform bounds on the the $L^{2}$ norm of some negative fractional derivative of $K$ restricted to the leaves of the foliation. This important latter result requires a commutator argument whose proof is postponed to the Appendix. We also provide proofs for our main $L^{2}$-elliptic estimates for Hodge systems.

In section 5 we describe our main sharp, Besov, bilinear trace theorems and Besov product results needed in the proof of the main theorem. Most results stated in this section require complicated arguments based on the geometric LP-theory developed in [Kl-Rodn3]-[Kl-Rodn4].

In section 6 we describe the structure and the estimates of the main commutator terms encountered in the proof of the main theorem.

In section 7 we rely on the results of previous sections to complete the proof of the main theorem.

In the Appendix we recall the definition of fractional powers of the Laplace-Beltrami operator on the leaves of the geodesic foliation of $\mathcal{H}$, based on the heat flow approach developed in [Kl-Rodn3], and provide the proof of the commutator estimate needed in section 4 .

\section{Null Hypersurfaces}

We recall in this section the basic geometric concepts associated to null hypersurface and refer the reader to $[\mathrm{Chr}-\mathrm{Kl}]$ and $[\mathrm{Kl}-\mathrm{Nic}]$ for a complete derivation of the null structure and null Bianchi identities.

We consider a null hypersurface $\mathcal{H}$ of an Einstein vacuum space-time $(\mathcal{M}, g)$. We assume that $\mathcal{H}$ is foliated by the level surfaces $S_{v}$ of a given function $v$ with $S_{0}$ fixed on an initial space-like hypersurface $\Sigma_{0}$. Let $L$ be the null geodesic generator of $\mathcal{H}$, i.e.

$$
<L, L>=0, \quad D_{L} L=0
$$

with $<,>=<,>_{g}$ denoting the metric of $\mathcal{M}$. At any point $P \in S_{v} \subset \mathcal{H}$ we denote by $\underline{L}$ the null vector conjugate to $L$ relative to the $S_{v}$ foliation, i.e.

$$
<L, \underline{L}>=-2, \quad<\underline{L}, X>=0 \quad \text { for all } X \in T_{p}\left(S_{v}\right) .
$$


We shall say that $L, \underline{L}$ form the canonical null pair associated to the foliation.

Remark 2.1. Observe that the null pair is uniquely defined up to a function constant along the null geodesic generators of $\mathcal{H}$.

We shall denote by $\gamma$ the induced metric on $S_{v}$, by $\nabla$ the induced covariant derivative and $K$ the Gauss curvature. An arbitrary orthonormal frame on $S_{v}$ will be denoted by $\left(e_{a}\right)_{a=1,2}$. Clearly,

$$
<e_{a}, L>=<e_{a}, \underline{L}>=0, \quad<e_{a}, e_{b}>=\delta_{a b} .
$$

A null pair together with an orthonormal frame $\left(e_{a}\right)_{a=1,2}$ as above is called a null frame associated to the foliation. We recall, see [Chr-Kl], [Kl-Nic], the definitions of the following basic geometric quantities:

Definition 2.2. The null second fundamental forms $\chi, \underline{\chi}$ of the foliation $S_{v}$ are given by

$$
\chi_{a b}=<D_{a} L, e_{b}>, \quad \underline{\chi}_{a b}=<D_{a} \underline{L}, e_{b}>
$$

The torsion is given by,

$$
\zeta_{a}=\frac{1}{2}<D_{a} L, \underline{L}>
$$

Remark 2.3. In view of the remark 2.1 we have the freedom to replace $L$, on the original surface $S_{0}$, by $L^{\prime}=\omega L$ where $\omega$ is an arbitrary scalar function on $S$. Therefore, choosing $\underline{L}^{\prime}=\omega^{-1} \underline{L}$ we have the transformation formulae,

$$
\chi^{\prime}=\omega \chi, \quad \underline{\chi}^{\prime}=\omega^{-1} \underline{\chi}, \quad \zeta^{\prime}=\zeta-\nabla \log \omega
$$

Definition 2.4. The null components of the curvature tensor $R$ of the space-time metric $g$ are given by:

$$
\begin{aligned}
\alpha_{a b} & =R\left(L, e_{a}, L, e_{b}\right), & & \beta_{a}=\frac{1}{2} R\left(e_{a}, L, \underline{L}, L\right), \\
\rho & =\frac{1}{4} R(\underline{L}, L, \underline{L}, L), & \sigma & =\frac{1}{4}{ }^{\star} R(\underline{L}, L, \underline{L}, L) \\
\underline{\beta}_{a} & =\frac{1}{2} R\left(e_{a}, \underline{L}, \underline{L}, L\right), & & \underline{\alpha}_{a b}=R\left(\underline{L}, e_{a}, \underline{L}, e_{b}\right)
\end{aligned}
$$

where ${ }^{\star} R$ denotes the Hodge dual of $R$. The null decomposition of ${ }^{\star} R$ can be related to that of $R$ according to the formulas, see[Chr-Kl] :

$$
\begin{aligned}
& \alpha\left({ }^{\star} R\right)=-{ }^{\star} \alpha(R), \quad \beta\left({ }^{\star} R\right)=-{ }^{\star} \beta(R), \quad \rho\left({ }^{\star} R\right)=\sigma(R) \\
& \sigma\left({ }^{\star} R\right)=-\rho(R), \quad \underline{\beta}\left({ }^{\star} R\right)=-{ }^{\star} \underline{\beta}(R), \quad \underline{\alpha}\left({ }^{\star} R\right)={ }^{\star} \underline{\alpha}(R)
\end{aligned}
$$

Observe that all tensors defined above are $S_{v}$-tangent, or simply S-tangent when there is no danger of confusion, i.e., tangent to the leaves of the $v$-foliation.

Definition 2.5. The particular case of the foliation defined by the level surfaces of the affine parameter $s$ of the null geodesic generator $L$ of $\mathcal{H}$, i.e.,

$$
L(s)=1,
$$


is called the geodesic(or background) foliation of $\mathcal{H}$. Given any other foliation $S_{v}$ we denote by

$$
\frac{d v}{d s}=\Omega_{v}=\Omega
$$

the null lapse of the $v$ - foliation.

To compare two arbitrary foliations it suffices to compare any one of them with the geodesic foliation $S_{s}$. Let $L, \underline{L}, e_{a}$ denote a null frame at $P \in \mathcal{H}$ for the geodesic foliation. Then the vectors $L, \underline{L}^{\prime}, e_{a}^{\prime}$ with

$$
e_{a}^{\prime}=e_{a}-\Omega^{-1} e_{a}(v) L, \quad \underline{L^{\prime}}=\underline{L}-2 \Omega^{-1} e_{a}(v) e_{a}-2 \Omega^{-2}|\nabla v|^{2} L .
$$

form a null frame for the $v$ foliation at $P$. Indeed, $e_{a}^{\prime}(v)=e_{a}(v)-\Omega^{-1} e_{a}(v) \frac{d v}{d s}=0$. Taking into account that $L$ is orthogonal to all tangent vectors to $\mathcal{H}$ we easily check that $<e_{a}^{\prime}, e_{b}^{\prime}>=<e_{a}, e_{b}>=\delta_{a b}$ and $<\underline{L}^{\prime}, \underline{L}^{\prime}>=<\underline{L}^{\prime}, e_{a}^{\prime}>=0$ while $<L, \underline{L^{\prime}}>=-2$.

Proposition 2.6. The null second fundamental form $\chi$ and curvature component $\alpha$ are intrinsic to the null hypersurface $\mathcal{H}$, i.e. they do not change by passing from one foliation to another.

$$
\chi_{a b}^{\prime}=\chi_{a b}, \quad \alpha_{a b}^{\prime}=\alpha_{a b}
$$

The torsion $\zeta$ verifies the following transformation formula

$$
\zeta_{a}^{\prime}=\zeta_{a}-\Omega^{-1} \chi_{a b} e_{b}(v)
$$

The curvature component $\beta$ verifies,

$$
\beta_{a}^{\prime}=\beta_{a}-\Omega^{-1} e_{b}(v) \alpha_{a b}
$$

Similarly $\rho^{\prime}-\rho, \sigma^{\prime}-\sigma$ can be expressed as linear combinations of $\alpha, \beta$ with coefficients depending on $\Omega^{-1} \nabla v$ and $\underline{\beta}^{\prime}-\underline{\beta}$ as a linear combination of $\alpha, \beta, \rho, \sigma$.

Proof : Indeed in view of the relations (20) between the frames $\underline{L}^{\prime}, e_{1}^{\prime}, e_{2}^{\prime}$ and $\underline{L}, e_{1}, e_{2}$ and the fact that $L$ is null geodesic,

$$
\begin{aligned}
\chi_{a b}^{\prime} & =<D_{e_{a}^{\prime}} L, e_{b^{\prime}}>=<D_{e_{a}} L+\Omega^{-1} e_{a}(v) D_{L} L, e_{b}+\Omega^{-1} e_{a}(v) L>=\chi_{a b} \\
2 \zeta_{a}^{\prime} & =<D_{e_{a}^{\prime}} L, \underline{L}^{\prime}>=<D_{e_{a}} L, \underline{L}^{\prime}>=<D_{a} L, \underline{L}>-2 \Omega^{-2} e_{b}(v)<D_{a} L, e_{b}> \\
& =2 \zeta_{a}-2 \Omega^{-1} \chi_{a b} e_{b}(v)
\end{aligned}
$$

as desired. To check the invariance of the null components of the curvature tensor we write with the help of (20),

$$
\begin{aligned}
\alpha_{a b}^{\prime} & =R\left(L, e_{a}^{\prime}, L, e_{b}^{\prime}\right)=R\left(L, e_{a}, L, e_{b}\right)=\alpha_{a b} \\
\beta_{a}^{\prime} & =\frac{1}{2} R\left(e_{a}^{\prime}, L, \underline{L}^{\prime}, L\right)=\frac{1}{2} R\left(e_{a}, L, \underline{L}-2 \Omega^{-1} e_{b}(v) e_{b}, L\right)=\beta_{a}-\Omega^{-1} e_{b}(v) \alpha_{a b} \\
\rho^{\prime} & =\frac{1}{4} R\left(\underline{L}^{\prime}-2 \Omega^{-1} e_{a}(v) e_{a}, L, \underline{L}^{\prime}-2 \Omega^{-1} e_{b}(v) e_{b}, L\right) \\
& =\rho-2 \Omega^{-1} e_{a}(v) \beta_{a}+\Omega^{-2} E_{a}(v) e_{b}(v) \alpha_{a b} \\
\sigma^{\prime} & =\frac{1}{4}^{\star} R\left(\underline{L}^{\prime}-2 \Omega^{-1} e_{a}(v) e_{a}, L, \underline{L}^{\prime}-2 \Omega^{-1} e_{b}(v) e_{b}, L\right) \\
& =\sigma+2 \Omega^{-1} e_{a}(v)^{\star} \beta_{a}-\Omega^{-2} E_{a}(v) e_{b}(v)^{\star} \alpha_{a b}
\end{aligned}
$$


The last equality holds in view of the formulas, see definition 2.4 , relating the null decomposition of $R$ to that of its Hodge dual ${ }^{\star} R$. We observe also that the Hodge dual is invariant relative to (20), i.e. $\in_{L L^{\prime} e_{1}^{\prime} e_{2}^{\prime}}=\in_{L \underline{L} e_{1} e_{2}}$. The transformation formula for $\underline{\beta}$ can be derived in the same manner.

We define now the other connection coefficient associated to an arbitrary $v$-foliation:

$$
\underline{\eta}_{a}=\frac{1}{2}<e_{a}, D_{L} \underline{L}>
$$

Proposition 2.7. The Ricci coefficient $\underline{\eta}$ associated to an arbitrary $v$-foliation verifies:

$$
\underline{\eta}_{a}=-\zeta_{a}+e_{a}(\log \Omega)=-\zeta_{a}+e_{a}(\log \Omega)
$$

Proof : Consider the commutator $\left[L, e_{a}\right]$. We claim that

$$
\left[L, e_{a}\right]=-e_{a}(\log \Omega) L+X
$$

where $X$ is a vectorfield tangent to $S_{v}$. Indeed, this follows easily from the fact that

Therefore,

$$
\left[L, e_{a}\right](v)=L e_{a}(v)-e_{a} L(v)=-e_{a}(\Omega)
$$

$$
\begin{aligned}
2 \underline{\eta}_{a} & =<e_{a}, D_{L} \underline{L}>=-<D_{L} e_{a}, \underline{L}>=-<D_{e_{a}} L, \underline{L}>-<\left[L, e_{a}\right], \underline{L}> \\
& =-2 \zeta_{a}+2 e_{a}(\log \Omega)
\end{aligned}
$$

as desired.

Definition 2.8. The S-tensors $\chi, \zeta, \underline{\chi}, \underline{\eta}$ form the connection coefficients of the $v-$ foliation. The following Ricci equations hold true:

$$
\begin{aligned}
& D_{a} \underline{L}=\underline{\chi}_{a b} e_{b}+\zeta_{a} \underline{L}, \quad D_{a} L=\chi_{a b} e_{b}-\zeta_{a} L \\
& D_{L} \underline{L}=2 \underline{\eta}_{b} e_{b}, \quad D_{L} L=0 \\
& D_{a} e_{b}=\nabla_{a} e_{b}+\frac{1}{2} \chi_{a b} \underline{L}+\frac{1}{2} \underline{\chi}_{a b} L
\end{aligned}
$$

Also,

$$
D_{L} e_{a}=\nabla_{L} e_{a}+\underline{\eta}_{a} L
$$

Definition 2.9. In general, given any tensor $\pi$ tangent to the leaves of the $v$ foliation, $\nabla_{L} \pi$ denotes the projection of $D_{L} \pi$ on $S_{v}$. Clearly, $\nabla_{L} \gamma=0$. A frame $\left(e_{a}\right)_{a=1,2}$ is said to be Fermi propagated if $\nabla_{L} e_{a}=0$.

Definition 2.10. Given an S-tangent one form $F$ we denote by ${ }^{\star} F$ its Hodge dual ${ }^{\star} F_{a}=\epsilon_{a b} F_{b}$. Similarly if if $F$ is an S-tangent symmetric traceless 2-tensor we define the left and right Hodge duals ${ }^{\star} F_{a b}=\epsilon_{a c} F_{c b}, F_{a b}^{\star}=F_{a}^{c} \in_{c b}$ and observe that $F^{\star}=-{ }^{\star} F$. For both one forms and symmetric traceless S-tensors $F$ we have ${ }^{\star}\left({ }^{\star} F\right)=-F$. Given two S-tangent traceless symmetric(or one forms) tensors $F, \eta$ we denote by $F \wedge \eta$ the scalar product between $F$ and the Hodge dual of $\eta$. Thus for one forms, $(F \wedge \eta)=\left(F \cdot{ }^{\star} \eta\right)=\epsilon_{a b} F_{a} F_{b}$ and for symmetric traceless tensors, $(F \wedge \eta)=\left(F \cdot^{\star} \eta\right)=\epsilon_{a b} F_{a c} F_{b c}$ 
Definition 2.11. Given an S-tangent symmetric tensor $F$ we denote by $\operatorname{tr} F=$ $\delta^{a b} F_{a b}$ its trace and by $\hat{F}$ its traceless part, i.e. $\hat{F}_{a b}=F_{a b}-\frac{1}{2}(\operatorname{tr} F) \delta_{a b}$

2.12. Structure equations for null-foliations. We shall use the general formulation of [Chr-Kl]. With the notation used on page 147 of [Chr-Kl] the full parameters of the $v$ foliation verify the following:

$$
H=\chi, \bar{H}=\underline{\chi}, Y=0, \bar{Z}=\underline{\eta}, V=\zeta, \Omega=0
$$

The structure equations of the $v$-foliation are( in view of the formula on pages 168-169 of [Chr-Kl]):

$$
\begin{aligned}
& L(\operatorname{tr} \chi)=-|\hat{\chi}|^{2}-\frac{1}{2}(\operatorname{tr} \chi)^{2} \\
& \nabla_{L} \hat{\chi}=-\operatorname{tr} \chi \cdot \hat{\chi}-\alpha \\
& \operatorname{div} \hat{\chi}=-\beta+\frac{1}{2} \nabla \operatorname{tr} \chi+\frac{1}{2} \operatorname{tr} \chi \zeta-\zeta \cdot \hat{\chi} \\
& L(\operatorname{tr} \underline{\chi})=2 \operatorname{div} \underline{\eta}+2 \rho-\frac{1}{2} \operatorname{tr} \chi \cdot \operatorname{tr} \underline{\chi}-\hat{\chi} \cdot \underline{\hat{\chi}}+2|\underline{\eta}|^{2} \\
& \nabla_{L} \underline{\hat{\chi}}=\nabla \widehat{\otimes} \underline{\eta}-\frac{1}{2} \operatorname{tr} \bar{\chi} \cdot \underline{\hat{\chi}}-\frac{1}{2} \operatorname{tr} \underline{\chi} \cdot \hat{\chi}+\underline{\eta} \widehat{\otimes} \underline{\eta} \\
& \nabla_{L} \zeta=-\beta+\chi \cdot(-\zeta+\underline{\eta}) \\
& \operatorname{curl} \underline{\eta}=\frac{1}{2} \hat{\chi} \wedge \underline{\hat{\chi}}-\sigma \\
& K=-\frac{1}{4} \operatorname{tr} \chi \operatorname{tr} \underline{\chi}+\frac{1}{2} \underline{\hat{\chi}} \cdot \hat{\chi}-\rho \\
& \operatorname{div} \underline{\hat{\chi}}=\frac{1}{2} \nabla \operatorname{tr} \underline{\chi}-\frac{1}{2} \operatorname{tr} \underline{\chi} \zeta+\zeta \cdot \underline{\hat{\chi}}+\underline{\beta}
\end{aligned}
$$

Recall also that $\underline{\eta}$ and $\zeta$ are related by the equation

$$
\underline{\eta}=-\zeta+\nabla \log \Omega
$$

where the null lapse $\Omega=\frac{d v}{d s}$ is a free parameter.

2.13. Null Bianchi equations. In view of the formulas on page 161 of [Chr-Kl] the Bianchi equations for $\beta, \rho, \sigma$ of the $v$-foliation are:

$$
\begin{aligned}
\nabla_{L} \beta+2 \operatorname{tr} \chi \beta & =\operatorname{div} \alpha+(2 \zeta+\underline{\eta}) \alpha \\
L(\rho)+\frac{3}{2} \operatorname{tr} \chi \rho & =\operatorname{div} \beta-\frac{1}{2} \underline{\hat{\chi}} \cdot \alpha+\zeta \cdot \beta+2 \underline{\eta} \cdot \beta \\
L(\sigma)+\frac{3}{2} \operatorname{tr} \chi \sigma & =-\operatorname{curl} \beta+\frac{1}{2} \underline{\hat{\chi}} \wedge \alpha-\zeta \wedge \beta-2 \underline{\eta} \wedge \beta \\
\nabla_{L} \underline{\beta}+\operatorname{tr} \chi \underline{\beta} & =-\nabla \rho+(\nabla \sigma)^{\star}+2 \underline{\hat{\chi}} \cdot \beta-3\left(\underline{\eta} \cdot \rho-\underline{\eta}^{\star} \sigma\right)
\end{aligned}
$$

2.14. Renormalized Null Bianchi. The presence of $\hat{\chi}$ on the right hand side (41), (42) (43) creates serious difficulties in the applications we have in mind. In what follows we remove this difficulty by introducing the renormalized null curvature components

$$
\check{\rho}=\rho-\frac{1}{2} \hat{\chi} \cdot \underline{\hat{\chi}}, \quad \check{\sigma}=\sigma-\frac{1}{2} \hat{\chi} \wedge \underline{\hat{\chi}}, \quad \underline{\check{\beta}}=\underline{\beta}+2 \underline{\hat{\chi}} \zeta
$$


Using the transport equations (33) and then (33) we notice that,

$$
\begin{aligned}
-\underline{\hat{\chi}} \cdot \alpha & =\underline{\hat{\chi}} \cdot\left(\nabla_{L} \hat{\chi}+\operatorname{tr} \chi \cdot \hat{\chi}\right) \\
& =L(\hat{\chi} \cdot \underline{\hat{\chi}})+\operatorname{tr} \chi \cdot \hat{\chi} \cdot \underline{\hat{\chi}}-\hat{\chi} \cdot\left(\nabla \widehat{\otimes} \underline{\eta}-\frac{1}{2} \operatorname{tr} \chi \underline{\hat{\chi}}-\frac{1}{2} \operatorname{tr} \underline{\chi} \cdot \hat{\chi}+\underline{\eta} \widehat{\otimes} \underline{\eta}\right)
\end{aligned}
$$

Thus,

$$
\begin{aligned}
L\left(\rho-\frac{1}{2} \hat{\chi} \cdot \underline{\hat{\chi}}\right)+\frac{3}{2} \operatorname{tr} \chi \cdot \rho & =\operatorname{div} \beta+\zeta \cdot \beta+2 \underline{\eta} \cdot \beta+\frac{1}{2} \operatorname{tr} \chi \hat{\chi} \cdot \underline{\hat{\chi}} \\
& -\frac{1}{2} \hat{\chi} \cdot\left(\nabla \widehat{\otimes} \underline{\eta}-\frac{1}{2} \operatorname{tr} \chi \underline{\hat{\chi}}-\frac{1}{2} \operatorname{tr} \underline{\chi} \cdot \hat{\chi}+\underline{\eta} \widehat{\otimes} \underline{\eta}\right)
\end{aligned}
$$

or

$$
\begin{aligned}
L\left(\rho-\frac{1}{2} \hat{\chi} \cdot \underline{\hat{\chi}}\right)+\frac{3}{2} \operatorname{tr} \chi \cdot\left(\rho-\frac{1}{2} \hat{\chi} \cdot \underline{\hat{\chi}}\right) & =\operatorname{div} \beta+(\zeta+2 \underline{\eta}) \cdot \beta \\
& -\frac{1}{2} \hat{\chi} \cdot\left(\nabla \widehat{\otimes} \underline{\eta}-\frac{1}{2} \operatorname{tr} \underline{\chi} \cdot \hat{\chi}+\underline{\eta} \widehat{\otimes} \underline{\eta}\right) .
\end{aligned}
$$

A similar equation holds for $\sigma$,

$$
\begin{aligned}
L\left(\sigma-\frac{1}{2} \hat{\chi} \wedge \underline{\hat{\chi}}\right)+\frac{3}{2} \operatorname{tr} \chi \cdot\left(\sigma-\frac{1}{2} \hat{\chi} \wedge \underline{\hat{\chi}}\right) & =-\operatorname{curl} \beta-(\zeta+2 \underline{\eta}) \wedge \beta \\
& -\frac{1}{2} \hat{\chi} \wedge(\nabla \widehat{\otimes} \underline{\eta}+\underline{\eta} \widehat{\otimes} \underline{\eta}) .
\end{aligned}
$$

We shall make a similar modification for the transport equation verified by $\beta$. The idea is to eliminate $2 \underline{\hat{\chi}} \cdot \beta$ from the right hand side of (43) with the help of (35) and (34). Indeed,

$$
\begin{aligned}
2 \underline{\hat{\chi}} \cdot \beta & =2 \underline{\hat{\chi}} \cdot\left(-\nabla_{L} \zeta+\chi \cdot(-\zeta+\underline{\eta})\right. \\
& =-2 L(\underline{\hat{\chi}} \cdot \zeta)+2 \chi \cdot \underline{\hat{\chi}} \cdot(-\zeta+\underline{\eta})+2\left(\nabla_{L} \underline{\hat{\chi}}\right) \cdot \zeta \\
& =-2 L(\underline{\hat{\chi}} \cdot \zeta)+2\left(\nabla \widehat{\widehat{\otimes}} \underline{\eta}-\frac{1}{2} \operatorname{tr} \chi \cdot \underline{\hat{\chi}}-\frac{1}{2} \operatorname{tr} \underline{\chi} \cdot \hat{\chi}+\underline{\eta} \widehat{\otimes} \underline{\eta}\right) \cdot \zeta \\
& +2 \chi \cdot \underline{\hat{\chi}} \cdot(-\zeta+\underline{\eta})
\end{aligned}
$$

Hence,

$$
\begin{aligned}
\nabla_{L}(\underline{\beta}+2 \underline{\hat{\chi}} \cdot \zeta) & =-\nabla \rho+(\nabla \sigma)^{\star}+2(\nabla \widehat{\otimes} \underline{\eta}) \cdot \zeta-3\left(\underline{\eta} \cdot \rho-\underline{\eta}^{\star} \sigma\right)-\operatorname{tr} \chi \underline{\beta} \\
& +2 \zeta \cdot\left(-\frac{1}{2} \operatorname{tr} \chi \cdot \underline{\hat{\chi}}-\frac{1}{2} \operatorname{tr} \underline{\chi} \cdot \hat{\chi}+\underline{\eta} \widehat{\widehat{\nabla}} \underline{\eta}\right)+2 \chi \cdot \underline{\hat{\chi}} \cdot(-\zeta+\underline{\eta})
\end{aligned}
$$

\subsection{Commutation formulas.}

Proposition 2.16. Consider an arbitrary $k$-covariant, $S$-tangent vectorfield $F_{\underline{a}}=$ $F_{a_{1} \ldots a_{k}}$ Then,

$$
\begin{aligned}
\nabla_{L} \nabla_{b} F_{\underline{a}}-\nabla_{b} \nabla_{L} F_{\underline{a}} & =-\chi_{b c} \nabla_{c} F_{\underline{a}}+\left(\zeta_{b}+\underline{\eta}_{b}\right) \nabla_{L} F_{\underline{a}} \\
& +\sum_{i}\left(\chi_{a_{i} b} \underline{\eta}_{c}-\chi_{b c} \underline{\eta}_{a_{i}}-\epsilon_{a_{i} c}{ }^{\star} \beta_{b}\right) F_{a_{1} \ldots c \ldots a_{k}}
\end{aligned}
$$

In particular for scalars $f$,

$$
\nabla_{L} \nabla_{b} f-\nabla_{b} \nabla_{L} f=-\chi_{b c} \nabla_{c} f+\left(\zeta_{b}+\underline{\eta}_{b}\right) \nabla_{L} f
$$


Also, for a one form $F$,

$L(\operatorname{div} F)-\operatorname{div}\left(\nabla_{L} F\right)=-\chi \cdot \nabla F+(\zeta+\underline{\eta}) \cdot \nabla_{L} F-\frac{1}{2} \operatorname{tr} \chi \cdot \underline{\eta} \cdot f-\hat{\chi} \cdot \underline{\eta} \cdot F-\beta \cdot F$

Finally, for scalars $f$,

$$
\begin{aligned}
L(\Delta f)-\Delta(L f) & =-2 \chi \cdot \nabla^{2} f+2(\zeta+\underline{\eta}) \cdot \nabla L(f)+\left(\operatorname{div} \zeta+\operatorname{div} \underline{\eta}+|\zeta+\underline{\eta}|^{2}\right) L(f) \\
& +\left(\frac{1}{2} \operatorname{tr} \chi(\zeta+\underline{\eta})+\hat{\chi} \cdot(\zeta-\underline{\eta})+\nabla \operatorname{tr} \chi+\operatorname{tr} \chi \zeta\right) \nabla f
\end{aligned}
$$

Remark 2.17. In this paper we shall only make use of the above commutator formulas for geodesic foliations, in which case $\zeta+\underline{\eta}=0$ and therefore the terms involving $\nabla_{L}$ vanish.

Remark 2.18. In view of (50) one would expect the presence of a term of the form $\beta \cdot \nabla f$ on the right hand side of (51). This terms cancells however due to the Cdazzi equation for $\hat{\chi}$. of

2.19. Mass aspect function. Differentiating the equation (35) and using the commutation formula (48) we infer,

$$
\begin{aligned}
\nabla_{L}\left(\nabla_{b} \zeta_{a}\right) & =-\nabla_{b} \beta_{a}+\nabla_{b}\left(\chi_{a c}\left(-\zeta_{c}+\underline{\eta}_{c}\right)\right)-\chi_{b c} \nabla_{c} \zeta_{a} \\
& +\left(\zeta_{b}+\underline{\eta}_{b}\right) \cdot \nabla_{L} \zeta_{a}+\zeta_{c}\left(\chi_{a b} \underline{\eta}_{c}-\chi_{b c} \underline{\eta}_{a}-\epsilon_{a c}{ }^{\star} \beta_{b}\right)
\end{aligned}
$$

Contracting we derive,

$$
\begin{aligned}
L(\operatorname{div} \zeta) & =-\operatorname{div} \beta-(\zeta-\underline{\eta}) \cdot \operatorname{div} \chi+\chi \cdot \nabla(\underline{\eta}-2 \zeta)+(\zeta+\underline{\eta}) \cdot \nabla_{L} \zeta \\
& +\frac{1}{2} \operatorname{tr} \chi \zeta \cdot \underline{\eta}-\hat{\chi} \cdot \zeta \cdot \underline{\eta}-\zeta \cdot \beta \\
& =-\operatorname{div} \beta-(\zeta-\underline{\eta}) \cdot\left(\nabla \operatorname{tr} \chi+\frac{1}{2} \zeta \operatorname{tr} \chi-\beta-\zeta \cdot \hat{\chi}\right) \\
& +\chi \cdot \nabla(\underline{\eta}-2 \zeta)+(\zeta+\underline{\eta}) \cdot(-\beta+\chi \cdot(-\zeta+\underline{\eta})) \\
& +\frac{1}{2} \operatorname{tr} \chi \zeta \cdot \underline{\eta}-\hat{\chi} \cdot \zeta \cdot \underline{\eta}-\zeta \cdot \beta \\
& =-\operatorname{div} \beta-\zeta \cdot \beta-(\zeta-\underline{\eta}) \cdot \nabla \operatorname{tr} \chi+\chi \cdot \nabla(\underline{\eta}-2 \zeta)+\operatorname{tr} \chi\left(-\zeta \cdot \zeta+\zeta \cdot \underline{\eta}+\frac{1}{2} \underline{\eta} \cdot \underline{\eta}\right) \\
& +\hat{\chi} \cdot(-2 \zeta \cdot \underline{\eta}+\underline{\eta} \cdot \underline{\eta})
\end{aligned}
$$

In other words,

$$
\begin{aligned}
L(\operatorname{div} \zeta) & =-\operatorname{div} \beta-\zeta \cdot \beta-(\zeta-\underline{\eta}) \cdot \nabla \operatorname{tr} \chi+\operatorname{tr} \chi\left(\frac{1}{2} \operatorname{div} \underline{\eta}-\operatorname{div} \zeta\right)+\hat{\chi} \cdot\left(\frac{1}{2} \nabla \widehat{\otimes} \underline{\eta}-\nabla \widehat{\otimes} \zeta\right) \\
& +\operatorname{tr} \chi\left(-\zeta \cdot \zeta+\zeta \cdot \underline{\eta}+\frac{1}{2} \underline{\eta} \cdot \underline{\eta}\right)-\hat{\chi} \cdot\left(\zeta \widehat{\otimes} \underline{\eta}+\frac{1}{2} \underline{\eta} \widehat{\otimes} \underline{\eta}\right)
\end{aligned}
$$

Combining this with (45) and introducing the mass aspect function

$$
\tilde{\mu}=-\operatorname{div} \zeta+\frac{1}{2} \hat{\chi} \cdot \underline{\hat{\chi}}-\rho
$$


we derive the following:

$$
\begin{aligned}
-L(\tilde{\mu}) & =L(\operatorname{div} \zeta)+L\left(\rho-\frac{1}{2} \hat{\chi} \cdot \underline{\hat{\chi}}\right) \\
& =-\operatorname{div} \beta-\zeta \cdot \beta-(\zeta-\underline{\eta}) \cdot \nabla \operatorname{tr} \chi+\operatorname{tr} \chi\left(\frac{1}{2} \operatorname{div} \underline{\eta}-\operatorname{div} \zeta\right)+\hat{\chi} \cdot\left(\frac{1}{2} \nabla \widehat{\otimes} \underline{\eta}-\nabla \widehat{\otimes} \zeta\right) \\
& +\operatorname{tr} \chi\left(-\zeta \cdot \zeta+\zeta \cdot \underline{\eta}+\frac{1}{2} \underline{\eta} \cdot \underline{\eta}\right)-\hat{\chi} \cdot\left(\zeta \widehat{\otimes} \underline{\eta}+\frac{1}{2} \underline{\eta} \widehat{\otimes} \underline{\eta}\right) \\
& -\frac{3}{2} \operatorname{tr} \chi\left(\rho-\frac{1}{2} \hat{\chi} \cdot \underline{\hat{\chi}}\right)+\operatorname{div} \beta+(\zeta+2 \underline{\eta}) \cdot \beta \\
& -\frac{1}{2} \hat{\chi} \cdot\left(\nabla \widehat{\otimes} \underline{\eta}-\frac{1}{2} \operatorname{tr} \underline{\chi} \hat{\chi}+\underline{\eta} \widehat{\otimes} \underline{\eta}\right) \\
& =-\hat{\chi} \cdot(\nabla \widehat{\otimes} \zeta)+2 \underline{\eta} \cdot \beta-\frac{3}{2} \operatorname{tr} \chi\left(\rho-\frac{1}{2} \hat{\chi} \cdot \underline{\hat{\chi}}\right)-(\zeta-\underline{\eta}) \cdot \nabla \operatorname{tr} \chi+\operatorname{tr} \chi\left(\frac{1}{2} \operatorname{div} \underline{\eta}-\operatorname{div} \zeta\right) \\
& +\operatorname{tr} \chi\left(-\zeta \cdot \zeta+\zeta \cdot \underline{\eta}+\frac{1}{2} \underline{\eta} \cdot \underline{\eta}\right)-\hat{\chi} \cdot(\underline{\eta} \widehat{\otimes} \underline{\eta}+\zeta \widehat{\otimes} \underline{\eta})+\frac{1}{4} \operatorname{tr} \underline{\chi} \hat{\chi} \cdot \hat{\chi}
\end{aligned}
$$

We have thus proved the following

Proposition 2.20. The mass aspect $\tilde{\mu}$ verifies the following transport equation:

$$
\begin{aligned}
L(\tilde{\mu})+\operatorname{tr} \chi \tilde{\mu} & =\hat{\chi} \cdot(\nabla \widehat{\otimes} \zeta)-2 \underline{\eta} \cdot \beta+\operatorname{tr} \chi\left(\rho-\frac{1}{2} \hat{\chi} \cdot \hat{\chi}\right)+(\zeta-\underline{\eta}) \cdot \nabla \operatorname{tr} \chi \\
& -\frac{1}{2} \operatorname{tr} \chi \operatorname{div} \underline{\eta}-\operatorname{tr} \chi\left(-\zeta \cdot \zeta+\zeta \cdot \underline{\eta}+\frac{1}{2} \underline{\eta} \cdot \underline{\eta}\right) \\
& +\hat{\chi} \cdot(\zeta \widehat{\otimes} \underline{\eta}+\underline{\eta} \underline{\otimes} \underline{\eta})-\frac{1}{4} \operatorname{tr} \underline{\chi} \hat{\chi} \cdot \hat{\chi}
\end{aligned}
$$

This transport equation should be viewed in combination with the following Hodge elliptic system for $\zeta$ :

$$
\begin{aligned}
\operatorname{div} \zeta & =-\tilde{\mu}+\frac{1}{2} \hat{\chi} \cdot \underline{\hat{\chi}}-\rho \\
\operatorname{curl} \zeta & =-\operatorname{curl} \underline{\eta}=\sigma-\frac{1}{2} \hat{\chi} \wedge \underline{\hat{\chi}}
\end{aligned}
$$

2.21. Transport equation for $\nabla \operatorname{tr} \chi$. For future reference we also record the transport equation for $\nabla \operatorname{tr} \chi$. This can be easily derived by differentiating the transport equation (30) for $\operatorname{tr} \chi$ and then use the commutation formulas (48). We derive,

$$
\begin{aligned}
\nabla_{L}(\nabla \operatorname{tr} \chi) & =-\frac{3}{2} \operatorname{tr} \chi \cdot \nabla \operatorname{tr} \chi-\hat{\chi} \cdot \nabla \operatorname{tr} \chi-2 \hat{\chi} \cdot \nabla \hat{\chi} \\
& -(\zeta+\underline{\eta})\left(|\hat{\chi}|^{2}+\frac{1}{2}(\operatorname{tr} \chi)^{2}\right)
\end{aligned}
$$

2.22. Geodesic foliation. The simplest foliation of a null hypersurface $\mathcal{H}$ is given by the geodesic foliation $\Omega=1$. In view of proposition 2.7, see also (39), we infer that

$$
\underline{\eta}=-\zeta
$$


The structure equations of the geodesic foliation take the form,

$$
\begin{aligned}
& \frac{d}{d s} \operatorname{tr} \chi=-\frac{1}{2}(\operatorname{tr} \chi)^{2}-|\hat{\chi}|^{2} \\
& \nabla_{L} \hat{\chi}=-\operatorname{tr} \chi \cdot \hat{\chi}-\alpha \\
& \nabla_{L}(\nabla \operatorname{tr} \chi)=-\frac{3}{2} \operatorname{tr} \chi \cdot \nabla \operatorname{tr} \chi-\hat{\chi} \cdot \nabla \operatorname{tr} \chi-2 \hat{\chi} \cdot \nabla \hat{\chi} \\
& \nabla_{L} \zeta=-\operatorname{tr} \chi \zeta-2 \hat{\chi} \cdot \zeta-\beta \\
& \nabla_{L}(\nabla \operatorname{tr} \chi)=-\frac{3}{2} \operatorname{tr} \chi \cdot \nabla \operatorname{tr} \chi-\hat{\chi} \cdot \nabla \operatorname{tr} \chi-2 \hat{\chi} \cdot \nabla \hat{\chi} \\
& \frac{d}{d s} \operatorname{tr} \underline{\chi}=-\frac{1}{2} \operatorname{tr} \chi \operatorname{tr} \underline{\chi}-2 \operatorname{div} \zeta-\hat{\chi} \cdot \underline{\hat{\chi}}+2|\zeta|^{2}+2 \rho \\
& \nabla_{L} \underline{\hat{\chi}}=-\nabla \hat{\otimes} \zeta-\frac{1}{2}(\operatorname{tr} \underline{\chi} \cdot \underline{\hat{\chi}}+\operatorname{tr} \underline{\chi} \cdot \hat{\chi})+\zeta \widehat{\otimes} \zeta \\
& \operatorname{div} \hat{\chi}=\frac{1}{2} \nabla \operatorname{tr} \chi-\hat{\chi} \cdot \zeta-\beta \\
& \operatorname{curl} \zeta=-\frac{1}{2} \hat{\chi} \wedge \underline{\hat{\chi}}+\sigma \\
& \operatorname{div} \underline{\hat{\chi}}=\frac{1}{2} \nabla \operatorname{tr} \underline{\chi}-\frac{1}{2} \operatorname{tr} \underline{\chi} \zeta+\zeta \cdot \underline{\hat{\chi}}+\underline{\beta} \\
& K=-\frac{1}{4} \operatorname{tr} \chi \operatorname{tr} \underline{\chi}+\frac{1}{2} \hat{\chi} \cdot \underline{\hat{\chi}}-\rho .
\end{aligned}
$$

We also have the renormalized Bianchi identities,

$$
\begin{aligned}
\nabla_{L} \beta & =\operatorname{div} \alpha+\zeta \cdot \alpha \\
L(\check{\rho})+\frac{3}{2} \operatorname{tr} \chi \cdot \check{\rho} & =\operatorname{div} \beta-\zeta \cdot \beta+\frac{1}{2} \hat{\chi} \cdot\left(\nabla \widehat{\otimes} \zeta+\frac{1}{2} \operatorname{tr} \underline{\chi} \cdot \hat{\chi}-\zeta \widehat{\otimes} \zeta\right) . \\
L(\check{\sigma})+\frac{3}{2} \operatorname{tr} \chi \cdot \check{\sigma} & =-\operatorname{curl} \beta+\zeta \wedge \beta+\frac{1}{2} \hat{\chi} \wedge(\nabla \widehat{\otimes} \zeta-\zeta \widehat{\otimes} \zeta) \\
\nabla_{L}(\underline{\beta}) & =-\nabla \rho+(\nabla \sigma)^{\star}-2(\nabla \widehat{\otimes} \zeta) \cdot \zeta+3\left(\zeta \cdot \rho-\zeta^{\star} \sigma\right)-\operatorname{tr} \underline{\beta} \\
& +2 \zeta \cdot\left(-\frac{1}{2} \operatorname{tr} \chi \underline{\hat{\chi}}-\frac{1}{2} \operatorname{tr} \underline{\chi} \cdot \hat{\chi}+\zeta \widehat{\otimes} \zeta\right)-4 \chi \underline{\chi} \underline{\hat{\chi}} \cdot \zeta
\end{aligned}
$$

where

$$
\check{\rho}=\rho-\frac{1}{2} \hat{\chi} \cdot \underline{\hat{\chi}}, \quad \check{\sigma}=\sigma-\frac{1}{2} \hat{\chi} \wedge \underline{\hat{\chi}}, \quad \underline{\check{\beta}}=\underline{\beta}+2 \underline{\hat{\chi}} \cdot \zeta .
$$

The transport equation for the mass aspect function $\tilde{\mu}$ takes the form,

$$
\begin{aligned}
\frac{d}{d s} \tilde{\mu}+\operatorname{tr} \chi \tilde{\mu} & =2 \zeta \cdot \beta+\operatorname{tr} \chi\left(\rho-\frac{1}{2} \hat{\chi} \cdot \underline{\hat{\chi}}\right)+2 \zeta \cdot \nabla \operatorname{tr} \chi+\frac{1}{2} \operatorname{tr} \chi \cdot \operatorname{div} \zeta+\hat{\chi} \cdot(\nabla \widehat{\otimes} \zeta) \\
& +\frac{3}{2} \operatorname{tr} \chi \zeta \cdot \zeta-\frac{1}{4} \operatorname{tr} \underline{\chi} \hat{\chi} \cdot \hat{\chi}
\end{aligned}
$$

This transport equation should be viewed in combination with the following Hodge elliptic system for $\zeta$ :

$$
\begin{aligned}
\operatorname{div} \zeta & =-\tilde{\mu}+\frac{1}{2} \hat{\chi} \cdot \underline{\hat{\chi}}-\rho=-\tilde{\mu}+\check{\rho} \\
\operatorname{curl} \zeta & =\sigma-\frac{1}{2} \hat{\chi} \wedge \underline{\hat{\chi}}=\check{\sigma}
\end{aligned}
$$


It turns out that the form of the transport equation (76) is not quite convenient in applications. To eliminate the curvature terms on the right hand side we observe, from the transport equation for $\zeta$,

$$
\frac{d}{d s}|\zeta|^{2}+2 \operatorname{tr} \chi|\zeta|^{2}=-4 \hat{\chi} \cdot \zeta \cdot \zeta-2 \zeta \cdot \beta
$$

Adding this to (76) and substituting $\operatorname{div} \zeta=-\tilde{\mu}-\check{\rho}$ we derive,

$$
\begin{aligned}
\frac{d}{d s} \mu+\frac{3}{2} \operatorname{tr} \chi \mu & =\hat{\chi} \cdot(\nabla \widehat{\otimes} \zeta)+\frac{1}{2} \operatorname{tr} \chi \check{\rho}+2 \zeta \cdot \nabla \operatorname{tr} \chi \\
& -4 \hat{\chi} \cdot \zeta \cdot \zeta+\operatorname{tr} \chi|\zeta|^{2}-\frac{1}{4} \operatorname{tr} \underline{\chi}|\hat{\chi}|^{2}
\end{aligned}
$$

where

$$
\mu=\tilde{\mu}+|\zeta|^{2}=-\operatorname{div} \zeta+\frac{1}{2} \hat{\chi} \cdot \underline{\hat{\chi}}-\rho+|\zeta|^{2}
$$

Remark 2.23. For a Fermi propagated frame $\left(e_{a}\right)_{a=1,2}$, i.e. $\nabla_{L} e_{a}=0$, we have $\left(\nabla_{L} \chi\right)_{a b}=\frac{d}{d s} \chi_{a b},\left(\nabla_{L} \zeta\right)_{a}=\frac{d}{d s} \zeta_{a}$ etc., and therefore all corresponding structure equations can be interpreted as differental equations along null geodesics.

Remark 2.24. As explained in Remark 2.3 we have the choice of selecting the null vector $L$ on the initial surface $S_{0}$. Passing from one fixed null pair $L, \underline{L}$ to another $L^{\prime}=\omega L, \underline{L^{\prime}}=\omega^{-1} \underline{L}$ we have $\hat{\chi}^{\prime}=\omega \hat{\chi}, \underline{\hat{\chi}}^{\prime}=\omega^{-1} \underline{\hat{\chi}}, \zeta^{\prime}=\zeta-\nabla \log \omega$. It is easy to check that the combination $\nabla \operatorname{tr} \chi+\zeta \operatorname{tr} \bar{\chi}$ remains invariant while $\tilde{\mu}^{\prime}=\tilde{\mu}+\Delta \log \omega$.

Remark 2.25. In view of the remark above it is thus possible to choose a null pair $L, \underline{L}$ relative to which the mass aspect function $\tilde{\mu}$ is constant on $S_{0}$. Indeed all we have to do is solve the equation $\Delta \log \omega=\tilde{\mu}-\overline{\tilde{\mu}}$, where $\overline{\tilde{\mu}}$ denotes the average of $\tilde{\mu}$ on $S_{0}$.

Finally we record below the following well known:

Lemma 2.26. Relative to the null geodesic foliation on $\mathcal{H}$,

$$
\frac{d}{d s} \int_{S_{s}} f d A_{s}=\int_{S_{s}}\left(\frac{d}{d s} f+\operatorname{tr} \chi f\right) d A_{s}
$$

where $d A_{s}=d A_{\gamma_{s}}$ denotes the volume element on $S_{s}$, with $\gamma_{s}=\gamma(s)$ the induced metric on $S_{s}$. In particular denoting by $r=r(s)=\sqrt{(4 \pi)^{-1}\left|S_{s}\right|}$, with $\left|S_{s}\right|$ the area of $S_{s}$, we have

$$
\frac{d r}{d s}=\frac{r}{2} \overline{\operatorname{tr} \chi}
$$

Proof : We parametrize the hypersurface $\mathcal{H}$ py picking arbitrary coordinates $\omega^{1}, \omega^{2}$ on $S_{0}$ and following the trajectories of the null generator vectorfield $L$. More precisely let $x^{\mu}=x^{\mu}\left(s, \omega^{1}, \omega^{2}\right)$ be the flow defined by:

$$
\frac{d x^{\mu}}{d s}=L^{\mu}
$$


with $x^{\mu}\left(0, \omega^{1}, \omega^{2}\right)$ the point on $S_{0}$ of coordinates $\left(\omega^{1}, \omega^{2}\right)$ and $s$ the affine parameter of $L$. We claim that relative to the coordinates $s, \omega^{1}, \omega^{2}$ on $\mathcal{H}$ the metric $\gamma_{s}$ verifies,

$$
\frac{d}{d s} \gamma_{a b}=2 \chi_{a b}
$$

Indeed relative to the coordinates $s, \omega^{1}, \omega^{2}$ on $\mathcal{H}$ we have $L=\frac{\partial}{\partial s}$ and since $\left[\frac{\partial}{\partial s}, \frac{\partial}{\partial \omega^{a}}\right]=0$ we infer from $\nabla_{L} \gamma=0$, and $\gamma_{a b}=\gamma\left(\frac{\partial}{\partial \omega^{a}}, \frac{\partial}{\partial \omega^{b}}\right)$,

$$
\begin{aligned}
0 & =\left(\nabla_{L} \gamma\right)\left(\frac{\partial}{\partial \omega^{a}}, \frac{\partial}{\partial \omega^{b}}\right)=\frac{d}{d s} \gamma_{a b}-\gamma\left(\nabla_{\frac{\partial}{\partial s}} \frac{\partial}{\partial \omega^{a}}, \frac{\partial}{\partial \omega^{b}}\right)-\gamma\left(\frac{\partial}{\partial \omega^{a}}, \nabla_{\frac{\partial}{\partial s}} \frac{\partial}{\partial \omega^{b}}\right) \\
& =\frac{d}{d s} \gamma_{a b}-\gamma\left(\nabla_{\frac{\partial}{\partial \omega^{a}}} L, \frac{\partial}{\partial \omega^{b}}\right)-\gamma\left(\frac{\partial}{\partial \omega^{a}}, \nabla_{\frac{\partial}{\partial \omega^{b}}} L\right) \\
& =\frac{d}{d s} \gamma_{a b}-2 \chi_{a b}
\end{aligned}
$$

as desired.

Therefore, denoting $|\gamma|=\operatorname{det}\left(\gamma_{a b}\right), \frac{d}{d s} \log |\gamma|=\gamma^{a b} \frac{d}{d s} \gamma_{a b}=2 \operatorname{tr} \chi \quad$ or,

$$
\frac{d}{d s} \sqrt{|\gamma|}=\operatorname{tr} \chi \sqrt{|\gamma|}
$$

Now, relative to the coordinates $s, \omega^{1}, \omega^{2}, \int_{S_{s}} f d \tilde{\mu}_{\gamma}=\iint f \sqrt{|\gamma|} d \omega^{1} d \omega^{2}$, therefore,

$$
\frac{d}{d s} \int_{S_{s}} f d A_{s}=\iint \frac{d}{d s}(f \sqrt{|\gamma|}) d \omega^{1} d \omega^{2}=\int_{S_{s}}\left(\frac{d}{d s} f+\operatorname{tr} \chi f\right) d A_{s}
$$

as desired.

Definition 2.27. We denote by $\gamma_{t}(\omega)$ the null geodesic $x^{\mu}\left(s, \omega^{2}, \omega^{2}\right) \subset \mathcal{H}$ initiating on $S_{0}$ at the point of coordinates $\omega=\left(\omega^{1}, \omega^{2}\right)$ and ending on $S_{t}$. Given a scalar function $f$ on $\mathcal{H}$ we denote by $\int_{\gamma_{t}(\omega)} f$, or simply $\int_{\gamma_{t}} f$ when no confusion is possible, the integral

$$
\int_{\gamma_{t}} f=\int_{\gamma_{t}} f d s=\int_{0}^{t} f\left(s, \omega^{1}, \omega^{2}\right) d s
$$

Definition 2.28. We denote by $\mathcal{H}_{t}$ the portion of the null hypersurface $\mathcal{H}$ corresponding to $0 \leq s \leq t$. Given a scalar function $f$ on $\mathcal{H}$ we denote by $\int_{\mathcal{H}_{t}} f$ the integral,

$$
\int_{\mathcal{H}_{t}} f=\int_{0}^{t} \int_{S_{s}} f d A_{s}=\int_{0}^{t} \iint f \sqrt{|\gamma|}\left(s, \omega^{1}, \omega^{2}\right) d s d \omega^{1} d \omega^{2}
$$

Remark 2.29. In view of the above definitions we easily check the co-area formula,

$$
\int_{\mathcal{H}_{t}} f=\int_{S_{0}}\left(\int_{\gamma_{t}} f \frac{\sqrt{\left|\gamma_{s}\right|}}{\sqrt{\left|\gamma_{0}\right|}} d s\right)
$$

Observe that $v_{s}=\frac{\sqrt{\left|\gamma_{s}\right|}}{\sqrt{\left|\gamma_{0}\right|}}$ verifies $\frac{d}{d s} v_{s}=\operatorname{tr} \chi v_{s}$ and therefore it can be used as an integrating factor according the formula,

$$
\nabla_{L}\left(v^{k} U\right)=v^{k}\left(\nabla_{L} U+k \operatorname{tr} \chi U\right)
$$


for arbitrary $S$-tangent tensors $U$. As a consequence of this formula, applied to scalar functions $\mathrm{f}$, we have:

Proposition 2.30. The equation $\frac{d}{d s} f+k \operatorname{tr} \chi f=g$, for scalars $f, g$ has the solution

$$
f(s, \omega)=v_{s}^{-k}\left(f(0, \omega)+\int_{0}^{s} v_{s^{\prime}}^{k} g\left(s^{\prime}, \omega\right) d s\right)
$$

In what follows we shall use lemma 2.26 to derive a transport equation for $\overline{\operatorname{tr} \chi}-\frac{2}{r}$. Using the transport equation (60) for tr $\chi$ and lemma 2.26 we derive,

$$
\begin{aligned}
\frac{d}{d s} \overline{\operatorname{tr} \chi} & =-\frac{2}{r} \frac{d r}{d s} \overline{\operatorname{tr} \chi}+\frac{1}{4 \pi r^{2}} \int_{S_{s}}\left(\frac{d}{d s} \operatorname{tr} \chi+\operatorname{tr} \chi^{2}\right) \\
& =-(\overline{\operatorname{tr} \chi})^{2}+\frac{1}{4 \pi r^{2}} \int_{S_{s}}\left(\frac{1}{2}(\operatorname{tr} \chi)^{2}-|\hat{\chi}|^{2}\right) \\
& =-(\overline{\operatorname{tr} \chi})^{2}+\frac{1}{2} \overline{(\operatorname{tr} \chi)^{2}}-\overline{|\hat{\chi}|^{2}} \\
& =-(\overline{\operatorname{tr} \chi})^{2}+\frac{1}{2} \overline{(\operatorname{tr} \chi-\overline{\operatorname{tr} \chi}+\overline{\operatorname{tr} \chi})^{2}}-\overline{|\hat{\chi}|^{2}} \\
& =-\frac{1}{2}(\overline{\operatorname{tr} \chi})^{2}+\frac{1}{2} \overline{(\operatorname{tr} \chi-\overline{\operatorname{tr} \chi})^{2}}-\overline{|\hat{\chi}|^{2}}
\end{aligned}
$$

On the other hand

$$
\frac{d}{d s}\left(2 r^{-1}\right)=-2 r^{-2} \frac{r}{2} \overline{\operatorname{tr} \chi}=-r^{-1} \overline{\operatorname{tr} \chi}
$$

Thus,

or,

$$
\begin{aligned}
\frac{d}{d s}\left(\overline{\operatorname{tr} \chi}-\frac{2}{r}\right) & =-\frac{1}{2}(\overline{\operatorname{tr} \chi})^{2}+\frac{1}{2} \overline{(\operatorname{tr} \chi-\overline{\operatorname{tr} \chi})^{2}}-\overline{|\hat{\chi}|^{2}}+\frac{1}{r} \overline{\operatorname{tr} \chi} \\
& =-\frac{1}{2} \overline{\operatorname{tr} \chi} \cdot\left(\overline{\operatorname{tr} \chi}-\frac{2}{r}\right)+\frac{1}{2} \overline{(\operatorname{tr} \chi-\overline{\operatorname{tr} \chi})^{2}}-\overline{|\hat{\chi}|^{2}}
\end{aligned}
$$

$$
\frac{d}{d s}\left(\overline{\operatorname{tr} \chi}-\frac{2}{r}\right)+\frac{1}{2}\left(\overline{\operatorname{tr} \chi}-\frac{2}{r}\right)=\frac{1}{2}\left((\operatorname{tr} \chi-\overline{\operatorname{tr} \chi})\left(\overline{\operatorname{tr} \chi}-\frac{2}{r}\right)+\overline{(\operatorname{tr} \chi-\overline{\operatorname{tr} \chi})^{2}}\right)-\overline{|\hat{\chi}|^{2}} .
$$

On the other hand,

$$
\begin{aligned}
\frac{d}{d s}(\operatorname{tr} \chi-\overline{\operatorname{tr} \chi}) & =-\frac{1}{2}\left(\operatorname{tr} \chi^{2}-(\overline{\operatorname{tr} \chi})^{2}\right)-\left(|\hat{\chi}|^{2}-\overline{|\hat{\chi}|^{2}}\right) \\
& =-\frac{1}{2}(\operatorname{tr} \chi-\overline{\operatorname{tr} \chi})(\operatorname{tr} \chi+\overline{\operatorname{tr} \chi})-\left(|\hat{\chi}|^{2}-\overline{|\hat{\chi}|^{2}}\right)
\end{aligned}
$$

Thus,

$$
\frac{d}{d s}(\operatorname{tr} \chi-\overline{\operatorname{tr} \chi})+\frac{1}{2} \operatorname{tr} \chi(\operatorname{tr} \chi-\overline{\operatorname{tr} \chi})=-\frac{1}{2} \overline{\operatorname{tr} \chi}(\operatorname{tr} \chi-\overline{\operatorname{tr} \chi})-\left(|\hat{\chi}|^{2}-\overline{|\hat{\chi}|^{2}}\right)
$$

We infer that,

Proposition 2.31. Let $W=\overline{\operatorname{tr} \chi}-\frac{2}{r}$ and $V=\operatorname{tr} \chi-\overline{\operatorname{tr} \chi}$. Then,

$$
\frac{d}{d s} W+\frac{1}{2} \operatorname{tr} \chi W=\frac{1}{2}\left(V \cdot W+V^{2}\right)-\overline{|\hat{\chi}|^{2}}
$$




$$
\frac{d}{d s} V+\frac{1}{2} \operatorname{tr} \chi V=-\frac{1}{2} \overline{\operatorname{tr} \chi} V-\left(|\hat{\chi}|^{2}-\overline{|\hat{\chi}|^{2}}\right)
$$

2.32. $L^{2}$ curvature norm associated to a geodesic foliation. Given a geodesic foliation on $\mathcal{H}$ and $F$ an arbitrary $S$-tensor on $\mathcal{H}$, and denoting by $\mathcal{H}_{t}$ the portion of $\mathcal{H}$ contained in the interval $s \in[0, t]$, we define its $L^{2}$ norm to be

$$
\|F\|_{L^{2}\left(\mathcal{H}_{t}\right)}=\left(\int_{0}^{t} d s \int_{S_{s}}|F|^{2}\right)^{\frac{1}{2}}
$$

where $\int_{S_{s}}|F|^{2}$ denotes the integral with respect to the volume element $d \tilde{\mu}_{s}$ of $S_{s}$. If $\mathcal{H}=\mathcal{H}_{1}$ we shall simply write $\|F\|_{L^{2}}=\|F\|_{L^{2}(\mathcal{H})}$.

Definition 2.33. Consider $\alpha, \beta, \rho, \sigma, \underline{\beta}$ to be the null decomposition of the curvature tensor relative to the geodesic foliation. We consider the norm:

$$
\mathcal{R}[t]=\left(\|\alpha\|_{L^{2}\left(\mathcal{H}_{t}\right)}^{2}+\|\beta\|_{L^{2}\left(\mathcal{H}_{t}\right)}^{2}+\|\rho\|_{L^{2}\left(\mathcal{H}_{t}\right)}^{2}+\|\sigma\|_{L^{2}\left(\mathcal{H}_{t}\right)}^{2}+\|\underline{\beta}\|_{L^{2}\left(\mathcal{H}_{t}\right)}^{2}\right)^{\frac{1}{2}}
$$

We say that the null hypersurface $\mathcal{H}=\mathcal{H}_{1}$ has bounded $L^{2}$ curvature flux, relative to the geodesic-foliation, if $\mathcal{R}_{0}=\mathcal{R}[1]<\infty$.

We shall also make us of the following notations:

Definition 2.34. Given an arbitrary $S$-tangent tensor $F$ on $\mathcal{H}=\mathcal{H}_{1}$ we denote

$$
\|\bar{\nabla} F\|_{L^{2}}=\|\nabla F\|_{L^{2}}+\left\|\nabla_{L} F\right\|_{L^{2}}
$$

We also introduce the following norms ,

$$
\begin{aligned}
\mathcal{N}_{1}(F) & =\|F\|_{L^{2}}+\|\bar{\nabla} F\|_{L^{2}} \\
& =\|F\|_{L^{2}}+\|\nabla F\|_{L^{2}}+\left\|\nabla_{L} F\right\|_{L^{2}} \\
\mathcal{N}_{2}(F) & =\|F\|_{L^{2}}+\|\bar{\nabla} F\|_{L^{2}}+\|\nabla \bar{\nabla} F\|_{L^{2}} \\
& =\|F\|_{L^{2}}+\|\nabla F\|_{L^{2}}+\left\|\nabla^{2} F\right\|_{L^{2}} \\
& +\left\|\nabla_{L} F\right\|_{L^{2}}+\left\|\nabla \cdot \nabla_{L} F\right\|_{L^{2}}
\end{aligned}
$$

We write $F \in \mathcal{N}_{1}(\mathcal{H})$ or $F \in \mathcal{N}_{2}(\mathcal{H})$, if $\mathcal{N}_{1}(F)<\infty$, resp. $\mathcal{N}_{2}(F)<\infty$.

2.35. Hodge systems. We consider the following Hodge operators acting on 2 surface $S$ diffeomorphic to the standard $S^{2}$ sphere, such as the leaves $S_{v}$ of a given foliation:

(1) The operator $\mathcal{D}_{1}$ takes any 1-form $F$ into the pairs of functions (div $F$, curl $F$ )

(2) The operator $\mathcal{D}_{2}$ takes any $S$ tangent symmetric, traceless tensor $F$ into the $S$ tangent one form div $F$.

(3) The operator ${ }^{\star} \mathcal{D}_{1}$ takes the pair of scalar functions $(\rho, \sigma)$ into the $S$-tangent 1-form $-\nabla \rho+(\nabla \sigma)^{\star}$. 
(4) The operator ${ }^{\star} \mathcal{D}_{2}$ takes 1-forms $F$ on $S$ into the 2-covariant, symmetric, traceless tensors $-\frac{1}{2} \widehat{\mathcal{L}_{F} \gamma}$ with $\mathcal{L}_{F} \gamma$ the traceless part of the Lie derivative of the metric $\gamma$ relative to $F$, i.e.

$$
{\widehat{\left(\mathcal{L}_{F} \gamma\right.}}_{a b}=\nabla_{b} F_{a}+\nabla_{a} F_{b}-(\operatorname{div} F) \gamma_{a b}
$$

Observe that the kernels of both $\mathcal{D}_{1}$ and $\mathcal{D}_{2}$ in $L^{2}(S)$ are trivial and that ${ }^{\star} \mathcal{D}_{1}$, resp. ${ }^{\star} \mathcal{D}_{2}$ are the $L^{2}$ adjoints of $\mathcal{D}_{1}$, respectively $\mathcal{D}_{2}$. The kernel of ${ }^{\star} \mathcal{D}_{1}$ consists of pairs of constant functions $(\rho, \sigma)$ while that of ${ }^{\star} \mathcal{D}_{2}$ consists of the set of all conformal Killing vectorfields on $S$. In particular the $L^{2}$ - range of $\mathcal{D}_{1}$ consists of all pairs of functions $\rho, \sigma$ on $S$ with vanishing mean curvature. The $L^{2}$ range of $\mathcal{D}_{2}$ consists of all $L^{2}$ integrable 1-forms on $S$ which are orthogonal to the Lie algebra of all conformal Killing vectorfields on $S$, see proposition 4.21. Accordingly we shall consider the inverse operators $\mathcal{D}_{1}^{-1}$ and $\mathcal{D}_{2}^{-1}$ and implicitly assume that they are defined on the $L^{2}$ subspaces identified above.

Finally we record the following simple identities,

$$
\begin{aligned}
{ }^{\star} \mathcal{D}_{1} \cdot \mathcal{D}_{1} & =-\Delta+K, \quad \mathcal{D}_{1} \cdot{ }^{\star} \mathcal{D}_{1}=-\Delta \\
{ }^{\star} \mathcal{D}_{2} \cdot \mathcal{D}_{2} & =-\frac{1}{2} \Delta+K, \quad \mathcal{D}_{2} \cdot{ }^{\star} \mathcal{D}_{2}=-\frac{1}{2}(\Delta+K)
\end{aligned}
$$

2.36. Null Structure equations in Minkowski space. In this section we show how to derive explicit formulas for the null structure equations in the flat case of the Minkowski space. These explicit formulas are well known, see for example [F-S].

We consider a null hypersurface $\mathcal{H}$ in Minkowski space. Let $S_{0} \subset \Sigma_{0}$ an initial 2 -surface with $\Sigma_{t}$ the standard space like hypersurfaces generated by $t$. Clearly $L=T+N, \underline{L}=T-N$ where $T$ denotes the unit normal to $\Sigma_{t}$ while $N$ is the exterior unit normal to $S_{t}=\Sigma_{t} \cap \mathcal{H}$ on $\Sigma_{t}$. Clearly the affine parameter $s$ of $L$ coincides with $t$. We also observe that $\zeta=0$ and $\chi=-\underline{\chi}=\theta$ where $\theta$ denotes the null second fundamental form of the surfaces $S_{t}$ with respect to $N$. Thus the geometry of $\mathcal{H}$ is entirely described by $\chi$, which we interpret here as $2 \times 2$ matrix with eigenvalues $\lambda$ and $\tilde{\mu}$, and we have the matrix equation,

$$
\frac{d}{d s} \chi=-\chi^{2}
$$

We look for solutions of (94) of the form $\chi(s)=U \cdot \Lambda(s) \cdot U^{-1}$ with $U$ independent of $s$ and $\Lambda$ diagonal. Therefore,

$$
\frac{d}{d s} \lambda=-\lambda^{2}, \quad \frac{d}{d s} \mu=-\mu^{2} .
$$

and we infer that,

$$
\lambda(s)=\frac{\lambda(0)}{1+s \lambda(0)}, \quad \mu(s)=\frac{\mu(0)}{1+s \mu(0)}
$$

Now let

$$
a=\operatorname{tr} \chi=\lambda+\mu, \quad b=\lambda \cdot \mu .
$$


Recall that $\chi^{2}-a \cdot \chi+b \cdot I=0$, with $I$ the identity matrix. On the other hand, since $\hat{\chi}=\chi-\frac{1}{2} a \cdot I$, we find

$$
|\hat{\chi}|^{2}=2\left(-b+\frac{1}{4} a^{2}\right)
$$

Now, in view of (95),

$$
\begin{aligned}
a(s) & =\frac{\lambda(0)}{1+s \lambda(0)}+\frac{\mu(0)}{1+s \mu(0)} \\
& =\frac{a(0)+2 s b(0)}{(1+s \lambda(0)) \cdot(1+s \mu(0))}
\end{aligned}
$$

and

$$
b(s)=\frac{\lambda(0)}{1+s \lambda(0)} \cdot \frac{\mu(0)}{1+s \mu(0)}=\frac{b(0)}{(1+s \lambda(0)) \cdot(1+s \mu(0))}
$$

Now, in view of (96), we easily calculate

$$
\begin{aligned}
-\frac{1}{2}|\hat{\chi}(s)|^{2} & =\left(b(s)-\frac{1}{4} a^{2}(s)\right)=\frac{4 b(0) \cdot \Delta(s)-(a(0)+2 s b(0))^{2}}{\Delta(s)^{2}} \\
& =\frac{4 b(0)-a(0)^{2}}{4 \Delta(s)}=-\frac{1}{2}|\hat{\chi}(0)|^{2} \cdot \frac{1}{\Delta(s)^{2}}
\end{aligned}
$$

where $\Delta(s)=(1+s \lambda(0)) \cdot(1+s \mu(0))=1+s a(0)+s^{2} b(0)$.

Therefore,

$$
|\hat{\chi}(s)|^{2}=\frac{|\hat{\chi}(0)|^{2}}{\Delta(s)^{2}}
$$

and we easily deduce that,

$$
\operatorname{tr} \chi(s)=\frac{\operatorname{tr} \chi(0)+s\left(\frac{1}{2} \operatorname{tr} \chi(0)^{2}-|\hat{\chi}(0)|^{2}\right)}{\Delta(s)}, \quad \hat{\chi}(s)=\frac{\hat{\chi}(0)}{\Delta(s)}
$$

It remains to identify the zeroes of $\Delta(s)$ i.e.

$$
s=-\lambda(0)^{-1}, \quad s=-\mu(0)^{-1} .
$$

Clearly $\lambda(0)+\mu(0)=\operatorname{tr} \chi(0), \lambda(0) \cdot \mu(0)=b(0)=\frac{1}{4} \operatorname{tr} \chi(0)^{2}-\frac{1}{2}|\hat{\chi}(0)|^{2}$. Thus,

$$
\lambda=\frac{\operatorname{tr} \chi(0)-\sqrt{2}|\hat{\chi}(0)|}{2}, \quad \mu=\frac{\operatorname{tr} \chi(0)+\sqrt{2}|\hat{\chi}(0)|}{2}
$$

Thus, for $s>0$, singularities only occur if $\lambda<0$ i.e.

$$
\operatorname{tr} \chi(0)<\sqrt{2}|\hat{\chi}(0)|
$$

In that case, for $s=s_{0}=-\lambda(0)^{-1}$, the numerator of $\operatorname{tr} \chi$ becomes

$$
a(0)+2 s_{0} b(0)=\lambda(0)+\mu(0)-2 \lambda(0)^{-1} \cdot \lambda(0) \cdot \mu(0)=\lambda(0)-\mu(0)
$$

which can only vanish if $\lambda(0)=\mu(0)$ i.e. $\hat{\chi}(0)=0$. Thus if $\hat{\chi}(0) \neq 0$ and condition (99) is satisfied then necessarily tr $\chi$ must become infinite at $s_{0}=-\lambda(0)^{-1}$. 
Consider now the volume element $d v_{s}=\sqrt{\left|\gamma_{s}\right|}$. Recall that, see (84), $\frac{d}{d s} \sqrt{|\gamma|}=$ $\operatorname{tr} \chi \sqrt{|\gamma|}$. Thus,

$$
\begin{aligned}
\frac{\sqrt{\left|\gamma_{s}\right|}}{\sqrt{\left|\gamma_{0}\right|}} & =\exp \int_{0}^{s}\left(\frac{\lambda(0)}{1+s^{\prime} \lambda(0)}+\frac{\mu(0)}{1+s^{\prime} \mu(0)}\right) d s^{\prime} \\
& =(1+s \lambda(0)) \cdot(1+s \mu(0))=\Delta(s)
\end{aligned}
$$

Now,

$$
\|\operatorname{tr} \chi\|_{L^{p}\left(S_{s}\right)}^{p}=\int_{S_{s}}|\operatorname{tr} \chi|^{p} d v_{s}=\int_{S_{0}} \frac{E^{p}(s)}{\Delta^{p}(s)} \Delta(s) d v_{0}=\int_{S_{0}} \frac{E^{p}(s)}{\Delta^{p-1}(s)} d v_{0}
$$

where $E(s)=\operatorname{tr} \chi(0)+s\left(\frac{1}{2} \operatorname{tr} \chi(0)^{2}-|\hat{\chi}(0)|^{2}\right)$. Similarily,

$$
\|\hat{\chi}\|_{L^{p}\left(S_{s}\right)}^{p}=\int_{S_{0}}|\hat{\chi}|^{p} \frac{1}{\Delta^{p-1}(s)} d v_{0}
$$

We infer that for all $p \geq 2$ the $L^{p}$ norms of $\operatorname{tr} \chi$ and $\hat{\chi}$ are infinite if the $L^{\infty}$ norms are. Hence we can not hope to control them unless we can control the $L^{\infty}$ norms.

\section{Motivation And Second version of the Main Theorem}

3.1. Further discussion of Main Theorem. Using the null structure and Bianchi equations discussed in the previous section we can now give a more detailed discussion of the main ideas underlining the proof of our main theorem. As in the introduction we write $\mathcal{H}=\mathcal{H}_{1}$ and $\mathcal{R}_{0}=\mathcal{R}[1]$.

Step 1. To start with we recall that tr $\chi$ verifies the transport equation

$$
\frac{d}{d s} \operatorname{tr} \chi=-|\hat{\chi}|^{2}-\frac{1}{2}(\operatorname{tr} \chi)^{2} .
$$

Integrating it we easily see that to bound tr $\chi$ it is necessary to have a uniform bound for $\int_{0}^{s}|\hat{\chi}|^{2}$. To obtain such an estimate we are allowed to rely only on the total curvature flux $\mathcal{R}_{0}$. The obvious idea which comes in mind is a trace theorem. One very much wishes that the following holds,

$$
\left(\int_{0}^{s}|\hat{\chi}|^{2}\right)^{\frac{1}{2}} \leq C\|\nabla \hat{\chi}\|_{L^{2}(\mathcal{H})}
$$

with a constant $C$ independent of $0 \leq s \leq 1$ and the corresponding null geodesic on which $|\hat{\chi}|^{2}$ is integrated. If such an estimate were true we could combine it with the Codazzi equation

$$
\operatorname{div} \hat{\chi}=-\beta+\frac{1}{2} \nabla \operatorname{tr} \chi+\frac{1}{2} \operatorname{tr} \chi \cdot \zeta-\zeta \cdot \hat{\chi}
$$

to estimate $\|\nabla \hat{\chi}\|_{L^{2}(\mathcal{H})}$ in terms of $\mathcal{R}_{0}$ using only $L^{2}$ elliptic estimates on the leaves $S_{s}$. Unfortunately this fails to be true. As explained in the introduction we could overcome this difficulty if $\hat{\chi}=\nabla_{L} Q+E$ with $Q$ a tensor verifying $\left\|\bar{\nabla}^{2} Q\right\|_{L^{2}}+$ 
$\|Q\|_{L^{2}} \lesssim \mathcal{R}_{0}$ and $E$ an appropriate error term. It turns out, miraculously, that if we combine the Codazzi equation with the null Bianchi identities (72), (73):

$$
\begin{aligned}
& L(\check{\rho})+\frac{3}{2} \operatorname{tr} \chi \cdot \check{\rho}=\operatorname{div} \beta-\zeta \cdot \beta+\frac{1}{2} \hat{\chi} \cdot\left(\nabla \widehat{\otimes} \zeta+\frac{1}{2} \operatorname{tr} \underline{\chi} \cdot \hat{\chi}-\zeta \widehat{\otimes} \zeta\right) . \\
& L(\check{\sigma})+\frac{3}{2} \operatorname{tr} \chi \cdot \check{\sigma}=-\operatorname{curl} \beta+\zeta \wedge \beta+\frac{1}{2} \hat{\chi} \wedge(\nabla \widehat{\otimes} \zeta-\zeta \widehat{\otimes} \zeta),
\end{aligned}
$$

we can obtain such a decomposition. Indeed, ignoring for now the quadratic and higher order terms in the Bianchi identities, we write,

$$
\mathcal{D}_{1} \beta=L(\check{\rho},-\check{\sigma})+\ldots
$$

On the other hand the Codazzi equation can be written in the form,

$$
\mathcal{D}_{2} \hat{\chi}=-\beta+\frac{1}{2} \nabla \operatorname{tr} \chi+\ldots
$$

Thus,

$$
\hat{\chi}=\mathcal{D}_{2}^{-1} \mathcal{D}_{1}^{-1} L(\check{\rho},-\check{\sigma})+\ldots
$$

or, ignoring the commutator term $\left[\mathcal{D}_{2}^{-1} \mathcal{D}_{1}^{-1}, \nabla_{L}\right](\check{\rho},-\check{\sigma})$, we can write,

$$
\hat{\chi}=L Q+\frac{1}{2} \mathcal{D}_{2}^{-1} \cdot \nabla \operatorname{tr} \chi+\ldots, \quad Q=\mathcal{D}_{2}^{-1} \cdot \mathcal{D}_{1}^{-1}(\check{\rho},-\check{\sigma})
$$

Using this expression for $\hat{\chi}$ we can return to (100) and write ${ }^{16}$,

$$
|\operatorname{tr} \chi| \lesssim \int_{0}^{s}|L Q|^{2}+\int_{0}^{s}\left|\mathcal{D}_{2}^{-1} \cdot \nabla \operatorname{tr} \chi\right|^{2}+\int_{0}^{s}|\operatorname{tr} \chi|^{2}+\int_{0}^{s} \mid \text { Error }\left.\right|^{2}
$$

In view of the trace theorem mentioned above we can hope to estimate, for sufficiently small $\mathcal{R}_{0}$,

$$
\left(\int_{0}^{s}|L Q|^{2}\right)^{\frac{1}{2}} \lesssim\left\|\bar{\nabla}^{2} \cdot \mathcal{D}_{2}^{-1} \cdot \mathcal{D}_{1}^{-1}(\check{\rho}, \check{\sigma})\right\|_{L^{2}(\mathcal{H})}+\|(\check{\rho}, \check{\sigma})\|_{L^{2}(\mathcal{H})} \lesssim \mathcal{R}_{0}
$$

All other terms, with the exception of $\int_{\gamma}\left|\mathcal{D}_{2}^{-1} \cdot \nabla \operatorname{tr} \chi\right|^{2}$, seem at first glance to be of lower order and thus controllable ${ }^{17}$. This last however leads to serious complications. Indeed $\mathcal{D}_{2}^{-1} \cdot \nabla$ is a nonlocal operator of order zero and therefore does not map $L^{\infty}$ into $L^{\infty}$. To overcome this difficulty we are forced to try to prove a stronger estimate for $\operatorname{tr} \chi$. The idea, as explained in the introduction, is to prove the boundedness of $\operatorname{tr} \chi$ not only in $L^{\infty}$ but rather in a Besov space of type $B_{2,1}^{1}\left(S_{s}\right)$ which imbeds in $L^{\infty}\left(S_{s}\right)$ and is stable under the action of $\mathcal{D}_{2}^{-1} \cdot \nabla$. Alternatively ${ }^{18}$ we can estimate $\nabla \operatorname{tr} \chi$ in a Besov norm of type $B_{2,1}^{0}\left(S_{s}\right)$. The transport character of the equations satisfied by $\operatorname{tr} \chi$ or $\nabla \operatorname{tr} \chi$ leads us to introduce the following modified Besov norms for $S$-tangent tensors $F, 0 \leq \theta \lesssim 1$ :

$$
\begin{aligned}
\|F\|_{\mathcal{B}^{\theta}} & =\sum_{k \geq 0} 2^{k \theta} \sup _{0 \leq s \leq 1}\left\|P_{k} F\right\|_{L^{2}\left(S_{s}\right)}+\sup _{0 \leq s \leq 1}\left\|P_{<0} F\right\|_{L^{2}\left(S_{s}\right)} \\
\|F\|_{\mathcal{P}^{\theta}} & =\sum_{k \geq 0} 2^{k \theta}\left\|P_{k} F\right\|_{L^{2}(\mathcal{H})}+\left\|P_{<0} F\right\|_{L^{2}(\mathcal{H})}
\end{aligned}
$$

\footnotetext{
${ }^{16}$ In reality we shall estimate $\operatorname{tr} \chi-\frac{2}{r}$. We choose to ignore this here to avoid additional technicalities.

${ }^{17}$ This is in fact not true, the commutator term $\left[\mathcal{D}_{2}^{-1} \mathcal{D}_{1}^{-1}, \nabla_{L}\right](\check{\rho},-\check{\sigma})$ turns out to have the same level of regularity as the principal terms. This fact forces us to treat all error terms very carefully in section 6 .

${ }^{18}$ This second approach is the one we shall actually take.
} 
with $P_{k}$ a family of intrinsic Littlewood-Paley projections which are properly defined, see [Kl-Rodn3], in terms of the heat flow on the surfaces $S_{s}$, see also [Stein]. Here ${ }^{19} P_{<0}=\sum_{k<0} P_{k}$. To show the boundedness of $\|\operatorname{tr} \chi\|_{B^{1}}$ we need:

(1) Prove a stronger trace type theorem of the form,

$$
\left\|\int_{0}^{s}|L Q|^{2}\right\|_{\mathcal{B}^{1}} \lesssim\left\|\bar{\nabla}^{2} Q\right\|_{L^{2}(H)}^{2}+\|Q\|_{L^{2}(H)}^{2} \lesssim \mathcal{R}_{0}^{2}
$$

(2) Show that

$$
\left\|\int_{0}^{s}\left|\mathcal{D}_{2}^{-1} \cdot \nabla \operatorname{tr} \chi\right|^{2}\right\|_{\mathcal{B}^{1}} \lesssim\|\operatorname{tr} \chi\|_{\mathcal{B}^{1}}^{2}
$$

(3) Construct the LP-projections $P_{k}$, intrinsic to the leaves of the foliation, with all desirable properties.

(4) Conclude, based on a bootstrap argument, that

$$
\|\operatorname{tr} \chi\|_{L^{\infty}(\mathcal{H})} \lesssim\|\operatorname{tr} \chi\|_{\mathcal{B}^{1}}, \quad\left\|\int_{0}^{1}|\hat{\chi}|^{2}\right\|_{L^{\infty}(\mathcal{H})}+\|\nabla \hat{\chi}\|_{L^{2}(\mathcal{H})}^{2} \lesssim \mathcal{R}_{0}^{2}
$$

(5) Make sure that all the error terms are controllable. It turns out in fact that the commutator $\left[\mathcal{D}_{2}^{-1} \mathcal{D}_{1}^{-1}, \nabla_{L}\right](\check{\rho},-\check{\sigma})$ is not in fact lower order, it has the same regularity properties as the main term $\hat{\chi}$ we have started with.

But this is not all. The error terms, denoted "Error" above, depend not only on $\operatorname{tr} \chi$ and $\hat{\chi}$ but also on $\zeta$. Moreover the renormalized curvature terms $\check{\rho}=\rho-\frac{1}{2} \hat{\chi} \cdot \hat{\chi}, \check{\sigma}=$ $\sigma-\frac{1}{2} \hat{\chi} \wedge \underline{\hat{\chi}}$ depend also on $\underline{\hat{\chi}}$ for which we have not yet any estimates. As we need to show that $\check{\rho}$ and $\check{\sigma}$ are bounded in $L^{2}(\mathcal{H})$ and, as we have explained below, we expect to be able to bound the sup norm of $\int_{0}^{1}|\hat{\chi}|^{2}$, we infer that we also need an estimate for $\left\|\sup _{0 \leq s \leq 1}|\underline{\chi}(s)|\right\|_{L^{2}\left(S_{0}\right)}$. To obtain such an estimate we need to consider the equations:

$$
\begin{aligned}
\frac{d}{d s} \operatorname{tr} \underline{\chi} & =-\frac{1}{2} \operatorname{tr} \underline{\operatorname{tr}} \underline{\chi}-2 \operatorname{div} \zeta-\hat{\chi} \cdot \underline{\hat{\chi}}+2|\zeta|^{2}+2 \rho \\
\operatorname{div} \underline{\hat{\chi}} & =\frac{1}{2} \nabla \operatorname{tr} \underline{\chi}-\frac{1}{2} \operatorname{tr} \underline{\chi} \zeta+\zeta \cdot \underline{\hat{\chi}}+\underline{\beta}
\end{aligned}
$$

from which we can easily convince ourselves that, to control $\left\|\sup _{0 \leq s \leq 1}|\underline{\chi}(s)|\right\|_{L^{2}\left(S_{0}\right)}$, we also need an $L^{2}(\mathcal{H})$ estimate for $\nabla \zeta$.

Step 2. To estimate $\nabla \zeta$ we use the transport equation (103) for $\mu$, which we write in the form

$$
\frac{d}{d s} \mu+\frac{3}{2} \operatorname{tr} \chi \mu=\hat{\chi} \cdot(\nabla \widehat{\otimes} \zeta)+\frac{1}{2} \operatorname{tr} \chi \check{\rho}+2 \zeta \cdot \nabla \operatorname{tr} \chi+\ldots
$$

together with the Hodge system (77) which we write in the form,:

$$
\mathcal{D}_{1} \zeta=-(\mu, 0)-(\check{\rho},-\check{\sigma})+\ldots
$$

To estimate $\|\nabla \zeta\|_{L^{2}(H)}$ we observe, by $L^{2}$ estimates,

$$
\|\nabla \zeta\|_{L^{2}(H)} \lesssim\left\|\mathcal{D}_{1} \zeta\right\|_{L^{2}(H)} \lesssim\|\mu\|_{L^{2}(\mathcal{H})}+\|\check{\rho}\|_{L^{2}}+\|\check{\sigma}\|_{L^{2}}
$$

\footnotetext{
${ }^{19}$ The low frequency component of the norms $\mathcal{B}^{\theta}, \mathcal{P}^{\theta}$, with $\theta \geq 0$, is much easier to treat and we shall often ignore it.
} 
To estimate ${ }^{20} \mu$ we write, integrating (103),

$$
\begin{aligned}
\left\|\sup _{s} \mu(s)\right\|_{L^{2}\left(S_{s}\right)} & \lesssim\|\mu(0)\|_{L^{2}\left(S_{0}\right)}+\left\|\int_{0}^{s} \hat{\chi} \cdot(\nabla \widehat{\otimes} \zeta)\right\|_{L^{2}\left(S_{s}\right)} \\
& +\left\|\int_{0}^{s} \zeta \cdot \nabla \operatorname{tr} \chi\right\|_{L^{2}\left(S_{s}\right)}+\ldots
\end{aligned}
$$

The first integral can be estimated with the help of,

$$
\left\|\int_{0}^{s} \hat{\chi} \cdot(\nabla \widehat{\otimes} \zeta)\right\|_{L^{2}\left(S_{s}\right)} \lesssim\left\|\left(\int_{0}^{s}|\hat{\chi}|^{2}\right)^{\frac{1}{2}}\right\|_{L^{\infty}\left(S_{s}\right)}\|\nabla \widehat{\otimes} \zeta\|_{L^{2}(\mathcal{H})}
$$

The second integral can be treated, since we expect to control $\left\|\sup _{t}|\nabla \operatorname{tr} \chi|\right\|_{L^{2}\left(S_{0}\right)}$, provided that we can control $\left\|\left(\int_{0}^{s}|\zeta|^{2}\right)^{\frac{1}{2}}\right\|_{L^{\infty}\left(S_{s}\right)}$. Thus to close we need to prove for $\zeta$ the same type of trace theorem as we have discussed above for $\hat{\chi}$. Yet, while $\zeta$ clearly plays the same role as $\hat{\chi}$ we do not have a quantity analogous to $\operatorname{tr} \chi$. Indeed the scalar $\mu$ is at the same level with $\nabla \operatorname{tr} \chi$ rather than $\operatorname{tr} \chi$.

The key observation for deriving trace type estimates for $\zeta$ is that the coupled transport-Hodge system for $(\mu, \zeta)$ has a similar structure to that of $(\nabla \operatorname{tr} \chi, \hat{\chi})$. Indeed taking a derivative of (100) we derive,

$$
\frac{d}{d s} \nabla \operatorname{tr} \chi=-\frac{3}{2} \operatorname{tr} \chi \cdot \nabla \operatorname{tr} \chi-\hat{\chi} \cdot \nabla \operatorname{tr} \chi-2 \hat{\chi} \cdot \nabla \hat{\chi}+\ldots
$$

The $B^{1}$ estimate for $\operatorname{tr} \chi$ is, at first glance ${ }^{21}$, equivalent to the $B^{0}$ estimate for $\nabla \operatorname{tr} \chi$. Using the transport equation for $\nabla \operatorname{tr} \chi$ we expect to show that ${ }^{22}$,

$$
\|\nabla \operatorname{tr} \chi\|_{\mathcal{B}^{0}} \lesssim\left\|\int_{0}^{s} \nabla \hat{\chi} \cdot \hat{\chi}\right\|_{\mathcal{B}^{0}}+\left\|\int_{0}^{s} \nabla \operatorname{tr} \chi \cdot \chi\right\|_{\mathcal{B}^{0}}
$$

To control the first integral we need to observe that

$$
\hat{\chi} \in \mathcal{N}_{1}(\mathcal{H}), \quad \nabla \hat{\chi}=\nabla_{L} P+\frac{1}{2} \nabla \cdot \mathcal{D}_{2}^{-1} \cdot \nabla \operatorname{tr} \chi+\text { Error }
$$

where $P \in \mathcal{N}_{1}(\mathcal{H})$. This replaces the previous decomposition of $\hat{\chi}$. In turn, the trace inequality (102) has to be replaced by a bilinear version ${ }^{23}$

$$
\left\|\int_{0}^{s} F \cdot \nabla_{L} P\right\|_{\mathcal{B}^{0}} \lesssim\left(\mathcal{N}_{1}(F)+\|F\|_{L_{x}^{\infty} L_{t}^{2}}\right) \cdot \mathcal{N}_{1}(P)
$$

The analogy between the systems for $(\mu, \zeta)$ and $(\nabla \operatorname{tr} \chi, \hat{\chi})$ suggests that we should look for a $\mathcal{B}^{0}$-Besov type estimate for $\mu$ and a bilinear trace inequality of the type (108) involving $\zeta$. To estimate $\left\|\int_{\gamma_{s}} \hat{\chi} \cdot \nabla \widehat{\otimes} \zeta\right\|_{\mathcal{B}^{0}}$ we first need to express $\nabla \zeta$ as we

\footnotetext{
${ }^{20}$ Note that there is no additional gain of regularity for $\|\mu\|_{L^{2}(\mathcal{H})}$.

${ }^{21}$ For technical reasons we cannot quite prove this equivalence. The main difference is that $M$ is a scalar quantity while $\nabla \operatorname{tr} \chi$ is a 1 -form.

${ }^{22} \mathrm{We}$ proceed quite formally here. In fact $\nabla \operatorname{tr} \chi$ is not a scalar and the integrals on the right hand side don't quite make sense. We only use this formal approach here to make a parallel with the estimates for $(\mu, \zeta)$, in which case $\mu$ is a scalar and all corresponding integrals make sense.

${ }^{23}$ This formulation is not quite appropriate since, in this case, $F \cdot \nabla_{L} P$ is a tensor and it does not quite make sense to take its integral, see previous footnote. We shall in fact only make use of this for scalars.
} 
did in (107) for $\nabla \hat{\chi}$. To achieve this we combine the Hodge system (104) with the null Bianchi identity ${ }^{24}$ connecting $\underline{\beta}$ with $(\rho, \sigma)$,

$$
\nabla_{L} \underline{\beta}=\mathcal{D}_{1}^{*}(\rho, \sigma)+2 \underline{\hat{\chi}} \cdot \beta+3\left(\zeta \cdot \rho-{ }^{*} \zeta \cdot \sigma\right)
$$

Thus setting $P=\mathcal{D}_{1}^{*-1} \underline{\beta}$, with $P \in \mathcal{N}_{1}(\mathcal{H})$, we can write,

$$
\nabla \zeta=\nabla_{L} P+\nabla \cdot \mathcal{D}_{1}^{-1}(-\mu, 0)+\ldots
$$

Now that we have established the formal similarity between the couples $(\nabla \operatorname{tr} \chi, \hat{\chi})$ and $(\mu, \zeta)$ we expect to be able to prove the $B^{0}$ estimate for $\mu$. Indeed, using the transport equation for $\mu$ as in (105) ${ }^{25}$,

$$
\|\mu\|_{\mathcal{B}^{0}} \lesssim\|\mu(0)\|_{\mathcal{B}^{0}}+\left\|\int_{0}^{s} \hat{\chi} \cdot(\nabla \widehat{\otimes} \zeta)\right\|_{\mathcal{B}^{0}}+\left\|\int_{0}^{s} \zeta \cdot \nabla \operatorname{tr} \chi\right\|_{\mathcal{B}^{0}}+\ldots
$$

The $B^{0}$ estimate for the integral $\int_{0}^{s} \chi \cdot(\nabla \widehat{\otimes} \zeta)$ uses the decomposition (109)

$$
\left\|\int_{0}^{s} \chi \cdot(\nabla \widehat{\otimes} \zeta)\right\|_{\mathcal{B}^{0}} \lesssim\left\|\int_{0}^{s} \hat{\chi} \cdot \nabla_{L} P\right\|_{\mathcal{B}^{0}}+\left\|\int_{0}^{s} \hat{\chi} \cdot \nabla \mathcal{D}_{1}^{-1}(\mu, 0)\right\|_{\mathcal{B}^{0}}+\ldots
$$

The first term can be estimated with the help of the bilinear trace inequality (108). The last term, since it does not contain an $L$-derivative, can be treated by means of the following bilinear inequality,

$$
\left\|\int_{0}^{s} F \cdot G\right\|_{\mathcal{B}^{0}} \lesssim\left(\mathcal{N}_{1}(F)+\left\|\left(\int_{0}^{s}|F|^{2}\right)^{\frac{1}{2}}\right\|_{L^{\infty}}\right)\|G\|_{\mathcal{P}^{0}}
$$

where $\mathcal{P}^{0}$ is the integrated Besov norm introduced in (113). This estimate forces us to control the $\mathcal{P}^{0}$-norm (which itself is dominated by the $\mathcal{B}^{0}$-norm) of $\nabla \cdot \mathcal{D}_{1}^{-1}(\mu, 0)$, which can be estimated by the $\mathcal{B}^{0}$-norm of $\mu$ and therefore can be absorbed by a bootstrap argument.

In addition, to treat the error terms we are required to control the $\mathcal{P}^{0}$-norm of the quantity $\hat{\chi} \cdot \underline{\hat{\chi}}$. We thus need yet another bilinear estimate of the form,

$$
\left.\|F \cdot G\|_{\mathcal{P}^{0}} \lesssim\left(\mathcal{N}_{1}(F)+\| \int_{0}^{s}|F|^{2}\right)^{\frac{1}{2}} \|_{L^{\infty}}\right)\|G\|_{\mathcal{B}^{0}}
$$

Thus to close our estimates we need a $B^{0}$-norm estimate for $\underline{\hat{\chi}}$.

Step 3. The idea is to derive a $B^{0}$ estimate for $\operatorname{tr} \underline{\chi}$ from the transport equation,

$$
\frac{d}{d s} \operatorname{tr} \underline{\chi}+\frac{1}{2} \operatorname{tr} \chi \operatorname{tr} \underline{\chi}=-2 \operatorname{div} \zeta-\hat{\chi} \cdot \underline{\hat{\chi}}+2|\zeta|^{2}+2 \rho .
$$

and combine it with elliptic estimates in $\mathcal{B}^{0}$ for the Hodge system,

$$
\operatorname{div} \underline{\hat{\chi}}=\frac{1}{2} \nabla \operatorname{tr} \underline{\chi}-\frac{1}{2} \operatorname{tr} \underline{\chi} \zeta+\zeta \cdot \underline{\hat{\chi}}+\underline{\beta} .
$$

\footnotetext{
${ }^{24}$ We shall in fact need the renormalized Bianchi identity (74).

${ }^{25}$ Remark that all the integrands on the right hand side are now scalars and thus the integrals along the null geodesics make sense.
} 
3.2. Sobolev and Besov type norms. Motivated by the discussion above we now give precise definitions of the Besov norms needed in our work. This requires the introduction of a family of Littlewood-Paley type projections $\left(P_{k}\right)_{k \in \mathbb{Z}}, \sum_{k} P_{k}^{2}=I$, verifying suitable properties. Such a family was defined in [Kl-Rodn3] with the help of the heat flow on the surfaces $S_{s}$. With the help of this family we define, in the usual way, the Besov norms $B_{2,1}^{\theta}$ on $S_{s}$. We also recall below the definition of the fractional Sobolev norms.

Definition 3.3. Given an arbitrary tensor $F$ on a fixed $S=S_{s}$ we define for every $\gamma \in \mathbb{R}$

$$
\|F\|_{H^{a}(S)}=\left\|\Lambda^{a} F\right\|_{L^{2}(S)} \approx\left(\sum_{k \geq 0} 2^{2 k a}\left\|P_{k} F\right\|_{L^{2}(S)}^{2}\right)^{\frac{1}{2}}+\left\|P_{<0} F(S)\right\|_{L^{2}}
$$

where $\Lambda=(I-\Delta)^{\frac{1}{2}}$, see definition in Appendix. We also define the Besov norms,

$$
\|F\|_{B_{2,1}^{a}(S)}=\left(\sum_{k \geq 0} 2^{a k}\left\|P_{k} F\right\|_{L^{2}(S)}\right)+\left\|\sum_{k<0} P_{k} F\right\|_{L^{2}(S)} .
$$

For $S$-tangent tensors $F$ on $\mathcal{H}=\mathcal{H}_{1}, 0 \leq \theta \leq 1$, we also introduce the norms:

$$
\begin{aligned}
\|F\|_{\mathcal{B}^{\theta}} & =\sum_{k \geq 0} 2^{k \theta} \sup _{0 \leq s \leq 1}\left\|P_{k} F\right\|_{L^{2}\left(S_{s}\right)}+\sup _{0 \leq s \leq 1}\left\|P_{<0} F\right\|_{L^{2}\left(S_{s}\right)}, \\
\|F\|_{\mathcal{P}^{\theta}} & =\sum_{k \geq 0} 2^{k \theta}\left\|P_{k} F\right\|_{L^{2}(\mathcal{H})}+\left\|P_{<0} F\right\|_{L^{2}(\mathcal{H})}
\end{aligned}
$$

3.4. Precise version of the Main Theorem. We start by making precise assumptions about the original 2-surface $S_{0} \subset \Sigma$.

Definition 3.5. Let $S_{0} \subset \Sigma$ be a compact 2-surface diffeomorphic to $\mathbf{S}^{2}$ with $\gamma$ a Riemannian metric on it. We say that $S_{0}$ is weakly regular if it can be covered by a finite number of coordinate charts $U$ with coordinates $\omega^{1}, \omega^{2}$ relative to which,

$$
\begin{gathered}
C^{-1}|\xi|^{2} \leq \gamma_{a b}(p) \xi^{a} \xi^{b} \leq c|\xi|^{2}, \quad \text { uniformly for all } p \in U \\
\sum_{a, b, c} \int_{U}\left|\partial_{c} \gamma_{a b}\right|^{2} d x^{1} d x^{2} \leq C^{-1}
\end{gathered}
$$

We say that the metric $\gamma$ is weakly spherical if in addition

$$
\sum_{a, b, c} \int_{U}\left|\partial_{c}\left(\gamma_{a b}-R^{2} \stackrel{\circ}{\gamma}_{a b}\right)\right|^{2} d x^{1} d x^{2} \leq I_{0}^{2}
$$

where $\stackrel{\circ}{\gamma}$ and is the standard metric on $\mathbf{S}^{2}$ and $R$ is a constant, $\frac{1}{2} \leq R \leq 2$. Here $I_{0}$ is a sufficiently small constant. We refer to surfaces, such as above, as WS surfaces.

Motivated by the discussion at the beginning of this section we are now ready to define the initial data quantity $\mathcal{I}_{0}$,

$$
\begin{aligned}
\mathcal{I}_{0} & =I_{0}+\left\|\operatorname{tr} \chi-\frac{2}{r}\right\|_{L^{\infty}\left(S_{0}\right)}+\|\nabla \operatorname{tr} \chi\|_{B_{2,1}^{0}\left(S_{0}\right)}+\|\mu\|_{B_{2,1}^{0}\left(S_{0}\right)} \\
& +\left\|\operatorname{tr} \underline{\chi}-\frac{2}{r}\right\|_{B_{2,1}^{0}\left(S_{0}\right)}+\|\underline{\hat{\chi}}\|_{B_{2,1}^{0}\left(S_{0}\right)}
\end{aligned}
$$

and give a precise version of our main result: 
Theorem 3.6 (Main Theorem). Consider an outgoing null hypersurface $\mathcal{H}=\mathcal{H}_{1}$, initiating on a compact 2 surface $S_{0} \subset \Sigma$ diffeomorphic to $\mathbf{S}^{2}$ and foliated by the geodesic foliation associated to the affine parameter $s$ with $\left.s\right|_{S_{0}}=0$. Assume that $r_{0}=r(0) \geq 1$ and that both the initial data quantity $\mathcal{I}_{0}$ and the total curvature flux $\mathcal{R}_{0}$ are sufficiently small. Then,

$$
\left\|\operatorname{tr} \chi-\frac{2}{r}\right\|_{L^{\infty}} \lesssim \mathcal{I}_{0}+\mathcal{R}_{0}
$$

Moreover,

$$
\begin{aligned}
\|\nabla \operatorname{tr} \chi\|_{\mathcal{B}^{0}}+\|\mu\|_{\mathcal{B}^{0}} & \lesssim \mathcal{I}_{0}+\mathcal{R}_{0} \\
\left\|\int_{0}^{1}|\hat{\chi}|^{2}\right\|_{L^{\infty}\left(S_{0}\right)}+\left\|\int_{0}^{1}|\zeta|^{2}\right\|_{L^{\infty}\left(S_{0}\right)} & \lesssim \mathcal{I}_{0}+\mathcal{R}_{0} \\
\left\|\sup _{0 \leq s \leq 1}|\nabla \operatorname{tr} \chi|\right\|_{L^{2}\left(S_{0}\right)}+\left\|\sup _{0 \leq s \leq 1}|\mu|\right\|_{L^{2}\left(S_{0}\right)} & \lesssim \mathcal{I}_{0}+\mathcal{R}_{0} \\
\mathcal{N}_{1}(\hat{\chi})+\mathcal{N}_{1}(\zeta)+\mathcal{N}_{1}\left(\operatorname{tr} \chi-\frac{2}{r}\right) & \lesssim \mathcal{I}_{0}+\mathcal{R}_{0} \\
\left\|\sup _{1 \leq s \leq 1}\left|\operatorname{tr} \underline{\chi}+\frac{2}{r}\right|\right\|_{L^{2}\left(S_{0}\right)}+\left\|\sup _{1 \leq s \leq 1}|\hat{\chi}|\right\|_{L^{2}\left(S_{0}\right)} & \lesssim \mathcal{I}_{0}+\mathcal{R}_{0} \\
\left\|\nabla_{L}\left(\operatorname{tr} \underline{\underline{\chi}}+\frac{2}{r}\right)\right\|_{L^{2}(\mathcal{H})} & \lesssim \mathcal{I}_{0}+\mathcal{R}_{0} \\
\left\|\nabla_{L} \underline{\hat{\chi}}\right\|_{L^{2}(\mathcal{H})} & \lesssim \mathcal{I}_{0}+\mathcal{R}_{0} \\
\left\|t r \underline{\chi}+\frac{2}{r}\right\|_{\mathcal{B}^{0}}+\|\underline{\hat{\chi}}\|_{\mathcal{B}^{0}} & \lesssim \mathcal{I}_{0}+\mathcal{R}_{0}
\end{aligned}
$$

The proof of the theorem relies on an elaborate bootstrap argument. In the next subsection we start by making a very mild boot-strap assumption and draw some simple important conclusions from it. The full set of bootstarp assumptions will be given in the next section. The idea is to prove that, by choosing the free constants $\mathcal{I}_{0}$ and $\mathcal{R}_{0}$, the bootstrap assumptions can be improved. The proof of the theorem then follows by a classical continuity argument.

3.7. Preliminary bootstrap assumption. We assume that there exists a sufficiently small positive constant $0<\Delta_{0}<\frac{1}{2}$ such that

BA1.

$$
\sup _{\mathcal{H}} r\left|\overline{\operatorname{tr} \chi}-\frac{2}{r}\right| \leq \Delta_{0}, \quad \sup _{\mathcal{H}} r|\operatorname{tr} \chi-\overline{\operatorname{tr} \chi}| \leq \Delta_{0} .
$$

We show later, at the completion of the proof that the same inequalities hold true with $\Delta_{0}$ replaced by $\frac{\Delta_{0}}{2}$.

In view of (81) we write,

$$
\frac{d r}{d s}=1+\frac{r}{2}\left(\overline{\operatorname{tr} \chi}-\frac{r}{2}\right)
$$


According to BA1. we infer by integration ${ }^{26}$,

$$
\begin{aligned}
\left|r(s)-r_{0}-s\right| & \leq\left|\int_{0}^{s} \frac{r}{2}\left(\overline{\operatorname{tr} \chi}-\frac{r}{2}\right)\right| \\
& \lesssim \frac{1}{2} s \Delta_{0}
\end{aligned}
$$

Thus, for $0 \leq s \leq t \leq 1$,

$$
r_{0}+\left(1-\frac{1}{2} \Delta_{0}\right) s \leq r(s) \leq r_{0}+\left(1+\frac{1}{2} \Delta_{0}\right) s
$$

or, simply since $\Delta_{0} \leq \frac{1}{2}$,

On the other hand,

$$
r_{0}+\frac{1}{2} s \leq r \leq r_{0}+\frac{3}{2} s
$$

$$
\left|\operatorname{tr} \chi-\frac{2}{r}\right| \leq \frac{2 \Delta_{0}}{r} .
$$

Hence, for all $0 \leq s \leq t$,

$$
\frac{2\left(1-\Delta_{0}\right)}{r_{0}+\left(1+\frac{1}{2} \Delta_{0}\right) s} \leq \operatorname{tr} \chi \leq \frac{2\left(1+\Delta_{0}\right)}{r_{0}+\left(1-\frac{1}{2} \Delta_{0}\right) s}
$$

Or, since $\Delta_{0}<\frac{1}{2}$,

$$
\frac{1}{r_{0}+2 s} \leq \operatorname{tr} \chi \leq \frac{3}{r_{0}+\frac{1}{2} s}
$$

Consequently,

$$
\log \frac{r_{0}+2 t}{r_{0}} \leq \int_{0}^{s} \operatorname{tr} \chi \leq 6 \log \frac{2 r_{0}+t}{2 r_{0}}
$$

As a consequence of the formula $\frac{d}{d s}(\log \sqrt{|\gamma|})=2 \operatorname{tr} \chi$ we infer that,

$$
v_{s}=\frac{\sqrt{\mid} \gamma_{s} \mid}{\sqrt{\mid} \gamma_{0} \mid}=\exp \left(2 \int_{0}^{s} \operatorname{tr} \chi\right)
$$

Therefore,

$$
\left(\frac{r_{0}+t}{r_{0}}\right)^{2} \leq \frac{\sqrt{\mid} \gamma_{s} \mid}{\sqrt{\mid} \gamma_{0} \mid} \leq\left(\frac{2 r_{0}+t}{2 r_{0}}\right)^{12}
$$

In particular since $r_{0} \geq 1,0 \leq t \leq 1 \leq r_{0}$,

$$
1 \leq v_{s}=\frac{\sqrt{\left|\gamma_{s}\right|}}{\sqrt{\left|\gamma_{0}\right|}} \leq 2\left(\frac{3}{2}\right)^{6}, \quad \text { for all } \quad 0 \leq s \leq t
$$

We infer that the volumes of $S_{s}$ and $S_{0}$ remain comparable for $0 \leq s \leq t$.

As a consequence of $(121)$ we also infer that the $L^{2}(\mathcal{H})$ norm, defined by the formula (90) is equivalent to the product norm on $[0, t] \times S_{0}$,

$$
\|F\|_{L^{2}}=\|F\|_{L_{t}^{2} L_{x}^{2}}=\left(\int_{0}^{1} \int_{S_{0}}|F|^{2} d A_{0} d s\right)^{\frac{1}{2}}=\left(\int_{0}^{1} d s \int_{S_{0}}|F(s, \omega)|^{2} \sqrt{\left|\gamma_{0}\right|} d \omega\right)^{\frac{1}{2}}
$$

\footnotetext{
${ }^{26}$ Recall that $r_{0}=r(0) \geq 1$.
} 
We shall also make use of the following norms,

$$
\begin{aligned}
& \|F\|_{L_{x}^{\infty} L_{t}^{2}}=\sup _{\omega \in S_{0}}\left(\int_{0}^{1} d s|F(s, \omega)|^{2}\right)^{\frac{1}{2}} \\
& \|F\|_{L_{x}^{2} L_{t}^{\infty}}=\left\|\sup _{0 \leq s \leq 1}|F(s, \omega)|\right\|_{L^{2}\left(S_{0}\right)}
\end{aligned}
$$

as well as, for $1 \leq p \leq \infty$,

$$
\begin{aligned}
\|F\|_{L_{t}^{\infty} L_{x}^{p}} & =\sup _{0 \leq s \leq 1}\|F(s)\|_{L^{p}\left(S_{0}\right)} \\
\|F\|_{L_{t}^{2} L_{x}^{p}} & =\left(\int_{0}^{1}\|F(s)\|_{L^{p}\left(S_{0}\right)}^{2} d s\right)^{\frac{1}{2}}
\end{aligned}
$$

Observe that $\|F\|_{L_{t}^{\infty} L_{x}^{2}} \leq\|F\|_{L_{x}^{2} L_{t}^{\infty}}$.

Lemma 3.8. Consider the equation $\nabla_{L} F+k \operatorname{tr} \chi F=G$ for $S$-tangent tensors $F, G$ on $\mathcal{H}$. Then, for any $1 \lesssim p \lesssim \infty$,

$$
\|F\|_{L_{x}^{p} L_{t}^{\infty}} \lesssim\|F(0)\|_{L^{p}\left(S_{0}\right)}+\|F\|_{L_{x}^{p} L_{t}^{1}}
$$

Proof : Multiplying the transport equation by $F$ we derive,

$$
\frac{d}{d s}|F|^{2}+2 k \operatorname{tr} \chi|F|^{2}=2 G \cdot F
$$

Applying to this formula (87) of proposition 2.30,

$$
|F(s, \omega)|^{2}=v(s, \omega)^{-2 k}\left(|F(0, \omega)|^{2}+\int_{0}^{s} v\left(s^{\prime}, \omega\right)^{2 k} G \cdot F\left(s^{\prime}, \omega\right) d s^{\prime}\right)
$$

where $v(s, \omega)=v_{s}(\omega)=\frac{\sqrt{\left|\gamma_{s}\right|}}{\sqrt{\left|\gamma_{0}\right|}}$. Using (121) we deduce,

$$
|F(s, \omega)|^{2} \lesssim|F(0, \omega)|^{2}+\int_{0}^{s}\left|G\left(s^{\prime}, \omega\right)\right|\left|F\left(s^{\prime}, \omega\right)\right| d s^{\prime}
$$

or, by a standard argument,

$$
|F(s, \omega)| \lesssim|F(0, \omega)|+\int_{0}^{s}\left|G\left(s^{\prime}, \omega\right)\right| d s^{\prime}
$$

We also have,

$$
\sup _{0 \leq s \leq 1}|F(s, \omega)| \lesssim|F(0, \omega)|+\int_{0}^{1}\left|G\left(s^{\prime}, \omega\right)\right| d s^{\prime}
$$

Thus the desired estimate (127) follows now by taking the $L^{p}$ norm with respect to $S_{0}$. 


\section{Boot-Strap assumptions. Preliminary estimates}

Based on the discussion of the previous section we make, in addition to BA1 the following stronger bootstrap assumptions for $\operatorname{tr} \chi, \hat{\chi}, \zeta$

BA2.

$$
\begin{aligned}
\|\hat{\chi}\|_{L_{x}^{\infty} L_{t}^{2}},\|\zeta\|_{L_{x}^{\infty} L_{t}^{2}} & \leq \Delta_{0} \\
\|\nabla \operatorname{tr} \chi\|_{L_{x}^{2} L_{t}^{\infty}},\|\mu\|_{L_{x}^{2} L_{t}^{\infty}} & \leq \Delta_{0} \\
\mathcal{N}_{1}(\hat{\chi}), \mathcal{N}_{1}(\zeta) & \leq \Delta_{0}
\end{aligned}
$$

BA3.

$$
\left\|\left(\operatorname{tr} \underline{\chi}+\frac{2}{r}\right)\right\|_{L_{x}^{2} L_{t}^{\infty}}, \quad\|\underline{\hat{\chi}}\|_{L_{x}^{2} L_{t}^{\infty}} \leq \Delta_{0}
$$

BA4.

$$
\left\|\operatorname{tr} \underline{\chi}+\frac{2}{r}\right\|_{\mathcal{B}^{0}}, \quad\|\underline{\hat{\chi}}\|_{\mathcal{B}^{0}} \leq \Delta .
$$

In the proof of theorem3.6 we shall show that, by chosing $\mathcal{I}_{0}$ and $\mathcal{R}_{0}$ small, we can recover the estimates above with $\Delta_{0}$ replaced by $\Delta_{0} / 2$.

Remark 4.1. Throughout this and the following section we shall only rely on BA1BA3. The assumption BA4 will be useful in the last section of the paper.

To simplify the discussion below it makes sense to introduce new notations which allow us to write in a schematic form the main structure and Bianchi equations, in view of the behavior of various components.

Definition 4.2. We introduce the following schematic notations for the curvature and connection coefficients.

(1) We denote by $R$ the full collection of null curvature components $\alpha, \beta, \rho, \sigma, \underline{\beta}$.

(2) We denote by $R_{0}$ the collection of null curvature components $\beta, \rho, \sigma, \underline{\beta}$.

(3) We denote by $\check{R}$ the collection of the renormalized null curvature components $(\check{\rho},-\check{\sigma}), \check{\beta}$, see $(75)$.

(4) We denote by $\bar{A}$ the collection $\operatorname{tr} \chi-\frac{2}{r}, \hat{\chi}, \zeta$.

(5) We denote by $\underline{A}$ the full collection $\left(\operatorname{tr} \chi-\frac{2}{r}\right), \hat{\chi}, \zeta$ as well as the "bad components" $\operatorname{tr} \underline{\chi}+\frac{2}{r}, \underline{\chi}$

(6) We denote by $M$ the collection $\nabla \operatorname{tr} \chi, \mu$.

(7) We denote by $\nabla A$ the collection formed by the first derivatives $\nabla \operatorname{tr} \chi, \nabla \hat{\chi}, \nabla \zeta$.

In view of these notations the bootstrap assumptions BA1 - BA4 take the form:

$$
\begin{aligned}
\|A\|_{L_{x}^{\infty} L_{t}^{2}} & \leq \Delta_{0}, \quad \mathcal{N}_{1}(A) \leq \Delta_{0} \\
\|M\|_{L_{x}^{2} L_{t}^{\infty}} & \leq \Delta_{0}, \quad\|\underline{A}\|_{L_{x}^{2} L_{t}^{\infty}} \leq \Delta_{0} \\
\|\underline{A}\|_{\mathcal{B}^{0}} & \leq \Delta_{0}
\end{aligned}
$$

We also have,

$$
\|R\|_{L_{t}^{2} L_{x}^{2}} \leq \mathcal{R}_{0}
$$


and assume that $\mathcal{R}_{0}$ is sufficiently small, in particular $\mathcal{R}_{0} \leq \Delta_{0}$. We record below some simple consequences of the bootstrap assumptions BA1-BA3.

4.3. Weakly spherical surfaces. We recall that the initial surface $S_{0}$ was weakly spherical WS in the sense of definition 3.5. We shall show, using BA1-BA2, that this property is shared by all surfaces $S_{s}$ of the geodesic foliation of $\mathcal{H}$. In other words all surfaces $S_{s}$ verify $\mathbf{W S}$.

Proposition 4.4. Consider the transported local coordinates $(s, \omega)$, defined by the equation $x^{\mu}=x^{\mu}(s, \omega)$ where $x^{\mu}(s, \omega)$ are null geodesics parametrized by the affine parameter $s$ and $\omega$ are local coordinates on $S_{0}$. Assume also that on $\left(S_{0}, \gamma\right)$ is weakly spherical in the sense of definition 3.5. Then, on all $\mathcal{H}$, the metric $\gamma$ remains weakly spherical, i.e.,

$$
\|\gamma\|_{L_{t}^{\infty} L_{x}^{\infty}},\left\|\gamma^{-1}\right\|_{L_{t}^{\infty} L_{x}^{\infty}} \lesssim 1, \quad\left\|\partial\left(\gamma-(1+s)^{2} \stackrel{\circ}{\gamma}\right)\right\|_{L_{x}^{2} L_{t}^{\infty}} \lesssim \mathcal{I}_{0}+\Delta_{0}
$$

Proof: We have already proved that the metric coefficients $\gamma$ remain bounded. To prove the second assertion we recall the equation (83),

$$
\frac{d}{d s} \gamma_{a b}=2 \chi_{a b} \text {. }
$$

Since the derivatices $\frac{\partial}{\partial \omega^{a}}$ commute with $\frac{d}{d s}$ and $\chi_{a b}=\frac{1}{2} \operatorname{tr} \chi \cdot \gamma_{a b}+\hat{\chi}_{a b}$ relative to the coordinate system $\omega^{1}, \omega^{2}$,

$$
\begin{aligned}
\frac{d}{d s} \partial_{c} \gamma_{a b} & =2 \partial_{c} \chi_{a b}=\partial_{c}\left(\operatorname{tr} \chi \cdot \gamma_{a b}+2 \hat{\chi}_{a b}\right) \\
& =\nabla_{c} \operatorname{tr} \chi \cdot \gamma_{a b}+\operatorname{tr} \chi \cdot \partial_{c} \gamma_{a b}+2 \partial_{c} \hat{\chi}_{a b} \\
& =+\operatorname{tr} \chi \cdot \partial_{c} \gamma_{a b}+\nabla_{c} \operatorname{tr} \chi \gamma_{a b}+2 \nabla_{c} \hat{\chi}_{a b}+(\partial \gamma) \cdot \hat{\chi}
\end{aligned}
$$

with $(\partial \gamma) \cdot \hat{\chi}$ denoting sum of terms involving only products between derivatives of the metric coefficients and components of $\hat{\chi}$. Therefore,

$$
\frac{d}{d s} \partial_{c} \gamma_{a b}-\operatorname{tr} \chi \cdot \partial_{c} \gamma_{a b}=\nabla_{c} \chi_{a b}+(\partial \gamma) \cdot \hat{\chi}
$$

According to proposition 2.30

$$
\partial \gamma(s, \omega)=v_{s}\left(\partial \gamma(0, \omega)+\int_{0}^{s} v_{s^{\prime}}^{-1} g\left(s^{\prime}, \omega\right) d s\right)
$$

where $g=\nabla \chi+(\partial \gamma) \cdot \hat{\chi}$, relative to the transported coordinates $\omega^{a}$. Therefore, denoting $\stackrel{\circ}{\gamma}_{s}=v_{s} \stackrel{\circ}{\gamma}=v_{s} \gamma(0)$ and observing that the components of $\partial \stackrel{\circ}{\gamma}_{s}$ are uniformly bounded,

$$
\begin{aligned}
\left\|\partial \gamma-\partial \dot{\gamma}_{s}\right\|_{L_{x}^{2} L_{t}^{\infty}} & \lesssim\|\nabla \chi\|_{L_{x}^{2} L_{t}^{1}}+\|(\partial \gamma) \cdot \hat{\chi}\|_{L_{x}^{2} L_{t}^{1}} \\
& \lesssim \Delta_{0}+\left\|\partial \gamma-\partial \dot{\gamma}_{s}\right\|_{L_{x}^{2} L_{t}^{\infty}} \cdot\|\hat{\chi}\|_{L_{x}^{\infty} L_{t}^{1}} \\
& \lesssim \Delta_{0}+\Delta_{0} \cdot\left\|\partial \gamma-\partial \dot{\gamma}_{s}\right\|_{L_{x}^{2} L_{t}^{\infty}}
\end{aligned}
$$

whence, in view of our weakly spherical assumption on $S_{0}$ and the smallness of $\Delta_{0}$,

$$
\left\|\partial\left(\gamma-\stackrel{\circ}{\gamma}_{s}\right)\right\|_{L_{x}^{2} L_{t}^{\infty}} \lesssim \Delta_{0}
$$


To end the proof of the theorem it only remains to remark that, for $0 \leq s \leq 1$, $\left|v_{s}-(1+s)^{2}\right| \lesssim \Delta_{0}$.

In particular our surfaces $S_{s}$ remain weakly regular. This fact allows us to apply the results of [Kl-Rodn3]. In particular we have the following calculus inequalities for scalar functions $f$ and tensorfields $F$ on each $S=S_{s} \subset \mathcal{H}$.

$$
\begin{aligned}
\|f\|_{L^{2}(S)} & \lesssim\|\nabla f\|_{L^{1}(S)}+\|f\|_{L^{1}(S)} \\
\|f\|_{L^{\infty}(S)} & \lesssim\left\|\nabla^{2} f\right\|_{L^{1}(S)}+\|f(S)\|_{L^{1}} \\
\|F\|_{L^{p}} & \lesssim\|\nabla F\|_{L^{2}}^{1-\frac{2}{p}}\|F\|_{L^{2}}^{\frac{2}{p}}+\|F\|_{L^{2}}, \quad 2 \leq p<\infty \\
\|F\|_{L^{\infty}} & \lesssim\left\|\nabla^{2} F\right\|_{L^{2}}^{\frac{1}{2}}\|F\|_{L^{2}}^{\frac{1}{2}}+\|F\|_{L^{2}}
\end{aligned}
$$

4.5. Basic Estimates on $\mathcal{H}$. The following simple calculus estimates hold true on $\mathcal{H}_{t}$, with any $0<t \leq 1$. In applications we shall consider only the case $t=1$.

Lemma 4.6. Let $F$ be an arbitrary $S$ tangent tensorfield on $\mathcal{H}=\mathcal{H}_{t}$. Then,

$$
\|F\|_{L_{t}^{\infty} L_{x}^{4}} \lesssim t^{-\frac{1}{2}}\|F\|_{L_{t}^{2} L_{x}^{4}}+\|F\|_{L_{x}^{\infty} L_{t}^{2}}^{\frac{1}{2}} \cdot\left\|\nabla_{L} F\right\|_{L_{t}^{2} L_{x}^{2}}^{\frac{1}{2}}
$$

Also,

$$
\begin{aligned}
\|F\|_{L_{t}^{\infty} L_{x}^{4}} \lesssim t^{-1 / 2}\left(\|F\|_{L_{t}^{2} L_{x}^{2}}+\|\nabla F\|_{L_{t}^{2} L_{x}^{2}}\right)+t^{1 / 2}\left\|\nabla_{L} F\right\|_{L_{t}^{2} L_{x}^{2}} \\
\|F\|_{L_{t}^{6} L_{t}^{6}} \lesssim t^{-1 / 3}\left(\|F\|_{L_{t}^{2} L_{x}^{2}}+\|\nabla F\|_{L_{t}^{2} L_{x}^{2}}\right)+t^{2 / 3}\left\|\nabla_{L} F\right\|_{L_{t}^{2} L_{x}^{2}} . \\
\|F\|_{L_{t}^{\infty} L_{x}^{\infty}} \lesssim t^{-\frac{1}{2}}\left(\|F\|_{L_{t}^{2} L_{x}^{2}}+\left\|\nabla^{2} F\right\|_{L_{t}^{2} L_{x}^{2}}\right) \\
+t^{\frac{1}{2}}\left(\left\|\nabla_{L} F\right\|_{L_{x}^{\infty} L_{t}^{2}}+\left\|\nabla \nabla_{L} F\right\|_{L^{2}}\right)
\end{aligned}
$$

Corollary 4.7. In the case of interest $t=1$ we have, see definition 2.34,

$$
\begin{aligned}
\|F\|_{L_{t}^{\infty} L_{x}^{4}}, \quad\|F\|_{L_{t}^{6} L_{x}^{6}} & \lesssim \mathcal{N}_{1}(F) \\
\|F\|_{L_{t}^{\infty} L_{x}^{\infty}} & \lesssim \mathcal{N}_{2}(F)
\end{aligned}
$$

Remark 4.8. The proof below provides in fact the stronger estimate,

$$
\|F\|_{L_{x}^{4} L_{t}^{\infty}} \lesssim \mathcal{N}_{1}(F) .
$$

Proof : Consider $|F|^{2}$. Integrating its derivative we write

$$
\begin{aligned}
|F(s, \omega)|^{2}-|F(0, \omega)|^{2} & =2 \int_{0}^{s} F \cdot \nabla_{L} F\left(s^{\prime}, \omega\right) d s^{\prime} \\
& \lesssim\left(\int_{0}^{s}\left|F\left(s^{\prime}, \omega\right)\right|^{2}\right)^{\frac{1}{2}} \cdot\left(\int_{0}^{s}\left|\nabla_{L} F\left(s^{\prime}, \omega\right)\right|^{2}\right)^{\frac{1}{2}}
\end{aligned}
$$

Therefore,

$$
\begin{aligned}
\left\||F(s)|^{2}-|F(0)|^{2}\right\|_{L^{2}\left(S_{0}\right)}^{2} & \lesssim \int_{S_{0}}\left(\int_{0}^{s}\left|F\left(s^{\prime}, \omega\right)\right|^{2}\right) \cdot\left(\int_{0}^{s}\left|\nabla_{L} F\left(s^{\prime}, \omega\right)\right|^{2}\right) \\
& \lesssim\|F\|_{L_{x}^{\infty} L_{t}^{2}}^{2} \cdot\left\|\nabla_{L} F\right\|_{L^{2}}^{2}
\end{aligned}
$$


It remains to estimate $\|F(0)\|_{L^{4}\left(S_{0}\right)}$. Let $0 \leq \theta(s) \leq 1$ be a test function on $[0, t]$ with $\theta(0)=1$, vanishing identically near $t$ and $\sup _{0 \leq s \leq t}\left|\theta^{\prime}(s)\right| \lesssim t^{-1}$. Then,

$$
\begin{aligned}
|F(0, \omega)|^{2} & =-\int_{0}^{t} \frac{d}{d s}\left(\theta(s)|F(s, \omega)|^{2}\right) d s \\
& \lesssim t^{-1} \int_{0}^{t}|F(s, \omega)|^{2}+\left(\int_{0}^{s}\left|F\left(s^{\prime}, \omega\right)\right|^{2}\right)^{\frac{1}{2}} \cdot\left(\int_{0}^{s}\left|\nabla_{L} F\left(s^{\prime}, \omega\right)\right|^{2}\right)^{\frac{1}{2}}
\end{aligned}
$$

Thus, taking $L^{2}$ norm in $S_{0}$ and applying Minkowski's inequality:

$$
\|F(0)\|_{L^{4}\left(S_{0}\right)} \lesssim t^{-\frac{1}{2}}\|F\|_{L_{t}^{2} L_{x}^{4}}+\|F\|_{L_{x}^{\infty} L_{t}^{2}}^{\frac{1}{2}} \cdot\left\|\nabla_{L} F\right\|_{L^{2}}^{\frac{1}{2}}
$$

Similarly, if we denote $|F|^{q}=\left(|F|^{2}\right)^{q / 2}$ for $q \geq 2$,

$$
\begin{aligned}
|F(0, \omega)|^{q} & =-\int_{0}^{t} \frac{d}{d s}\left(\theta(s)|F(s, \omega)|^{q}\right) d s \\
& \lesssim t^{-1}\|F\|_{L_{t}^{q} L_{x}^{q}}^{q}+\left\|\nabla_{L} F\right\|_{L_{t}^{2} L_{x}^{2}} \cdot\|F\|_{L_{t}^{2(q-1)} L_{x}^{2(q-1)}}^{q-1}
\end{aligned}
$$

Hence, reasoning as above, and by simple interpolation,

$$
\begin{aligned}
\|F\|_{L_{t}^{\infty} L_{x}^{q}} & \lesssim t^{-1 / q}\|F\|_{L_{t}^{q} L_{x}^{q}}+\left\|\nabla_{L} F\right\|_{L_{t}^{2} L_{x}^{2}}^{1 / q} \cdot\|F\|_{L_{t}^{2(q-1)} L_{x}^{2(q-1)}}^{(q-1) / q} \\
& \lesssim\left(\left(t^{-1}\|F\|_{L_{t}^{2} L_{x}^{2}}\right)^{1 / q}+\left\|\nabla_{L} F\right\|_{L_{t}^{2} L_{x}^{2}}^{1 / q}\right) \cdot\|F\|_{L_{t}^{2(q-1)} L_{x}^{2(q-1)}}^{(q-1) / q}
\end{aligned}
$$

which, for $q=4$, becomes

$$
\|F\|_{L_{t}^{\infty} L_{x}^{4}} \lesssim\left(t^{-1 / 4}\|F\|_{L_{t}^{2} L_{x}^{2}}^{1 / 4}+\left\|\nabla_{L} F\right\|_{L_{t}^{2} L_{x}^{2}}^{1 / 4}\right)\|F\|_{L_{t}^{6} L_{x}^{6}}^{3 / 4}
$$

On the other hand we have the classical Sobolev inequality ${ }^{27}$ on $\mathcal{H}$,

$$
\|F\|_{L_{t}^{6} L_{t}^{6}} \lesssim t^{-1 / 3}\left(\|F\|_{L_{t}^{2} L_{x}^{2}}+\|\nabla F\|_{L_{t}^{2} L_{x}^{2}}\right)+t^{2 / 3}\left\|\nabla_{L} F\right\|_{L_{t}^{2} L_{x}^{2}} .
$$

Therefore, combining with the previous inequality,

$$
\|F\|_{L_{t}^{\infty} L_{x}^{4}} \lesssim t^{-1 / 2}\left(\|F\|_{L_{t}^{2} L_{x}^{2}}+\|\nabla F\|_{L_{t}^{2} L_{x}^{2}}\right)+t^{1 / 2}\left\|\nabla_{L} F\right\|_{L_{t}^{2} L_{x}^{2}}
$$

which is precisely (139). The Sobolev inequality (141) follows from (139) combined with,

$$
\|F\|_{L^{4}(S)} \lesssim\|F\|_{L^{2}(S)}^{1 / 2} \cdot\left(\|\nabla F\|_{L^{2}(S)}+\|F\|_{L^{2}(S)}\right)^{1 / 2} .
$$

which is a particular case of (136).

Combining the results above with the bootstrap assumptions BA1-BA2 we deduce the following estimates for $A$ :

$$
\|A\|_{L_{t}^{\infty} L_{x}^{4}}, \quad\|A\|_{L_{t}^{6} L_{x}^{6}} \lesssim \Delta_{0}
$$

Also for $2 \leq p<\infty$,

$$
\|A\|_{L_{t}^{2} L_{x}^{p}} \lesssim \Delta_{0}
$$

${ }^{27}$ See section 4.1 of $[\mathrm{Kl}-\mathrm{Nic}$, also chapter 3 of [Chr-Kl] for a direct proof of this Sobolev inequality relying on the weak isoperimetric inequality (134). 
4.9. Estimates for $\|\check{R}\|_{L_{t}^{2} L_{r}^{2}}$. Using the bootstrap assumptions BA1-BA3 we can easily deduce the following:

Proposition 4.10. The renormalized curvature components $\check{R}$ verify:

$$
\|\check{R}\|_{L_{t}^{2} L_{x}^{2}} \leq \mathcal{R}_{0}+\Delta_{0}^{2}
$$

Proof : To prove (145) it suffices to check the assertion for $\check{\rho}=\rho-\frac{1}{2} \hat{\chi} \cdot \underline{\hat{\chi}}$, the other components are similar. Clearly,

$$
\|\check{\rho}\|_{L_{t}^{2} L_{x}^{2}} \lesssim\|\rho\|_{L_{t}^{2} L_{x}^{2}}+\|\hat{\chi}\|_{L_{x}^{\infty} L_{t}^{2}} \cdot\|\underline{\hat{\chi}}\|_{L_{x}^{2} L_{t}^{\infty}} \lesssim \mathcal{R}_{0}+\Delta_{0}^{2}
$$

4.11. Gauss Curvature. Recall that the Gauss curvature $K$ of $S_{s}$ is given by the formula, see (70),

$$
K=-\frac{1}{4} \operatorname{tr} \chi \operatorname{tr} \underline{\chi}+\frac{1}{2} \hat{\chi} \cdot \underline{\hat{\chi}}-\rho .
$$

or, writing schematically with the help of the definitions above, $\operatorname{tr} \chi=\frac{2}{r}+A$, $\operatorname{tr} \underline{\chi}=-\frac{2}{r}+\underline{A}$ and,

$$
K-\frac{1}{r^{2}}=\frac{1}{2 r} A-\frac{1}{2 r} \underline{A}-\frac{1}{4} A \cdot \underline{A}+\frac{1}{2} A \cdot \underline{A}+R .
$$

Since the term $\frac{1}{2 r} A$ is better than $\frac{1}{2 r} \underline{A}$ we may neglect it and, neglecting also signs and constants on the right hand side, we write

$$
K-\frac{1}{r^{2}}=\frac{1}{2 r} \underline{A}+A \cdot \underline{A}+R
$$

Thus, according to our bootstrap assumptions and $r \geq 1$,

$$
\begin{aligned}
\left\|K-\frac{1}{r^{2}}\right\|_{L_{t}^{2} L_{x}^{2}} & \lesssim\|\underline{A}\|_{L_{t}^{2} L_{x}^{2}}+\|A\|_{L_{x}^{\infty} L_{t}^{2}} \cdot\|\underline{A}\|_{L_{x}^{2} L_{t}^{\infty}}+\|R\|_{L_{t}^{2} L_{x}^{2}} \\
& \lesssim \Delta_{0}+\Delta_{0}^{2}+\mathcal{R}_{0}
\end{aligned}
$$

Thus,

This proves the the following

$$
\left\|K-\frac{1}{r^{2}}\right\|_{L_{t}^{2} L_{x}^{2}} \lesssim \Delta_{0}
$$

Proposition 4.12. The Gauss curvature $K$ of $S=S_{s} \subset \mathcal{H}$ verifies the following estimates,

$$
\left\|K-\frac{1}{r^{2}}\right\|_{L_{t}^{2} L_{x}^{2}} \lesssim \Delta_{0}
$$

We shall also need the following important:

Proposition 4.13. The following estimate holds for all $\frac{1}{2}<a<1$,

$$
\left\|\Lambda^{-a}\left(K-\frac{1}{r^{2}}\right)\right\|_{L_{t}^{\infty} L_{x}^{2}} \lesssim \Delta_{0}
$$

where $\Lambda=(I-\Delta)^{\frac{1}{2}}$. 
Proof: Fractional powers of the Laplacean $\Lambda^{a}=(1-\Delta)^{a / 2}$ on surfaces $S$ can be defined with the help of the heat flow on $S$. This approach has been developed in [Kl-Rodn3] based on an assumption of weak regularity. We note that this assumption is implied by the WS property derived in proposition 4.4. We refer the reader to the appendix for a quick review of the main properties of the heat flow needed in our proof of the proposition. We shall make repetead use of the composition properties of the operators $\Lambda^{a}$

$$
\Lambda^{a} \cdot \Lambda^{b}=\Lambda^{a+b}
$$

Note also that the operators $\Lambda^{a}$ are bounded in $L^{2}$ for $a \leq 0$. Thus,

$$
\left\|\Lambda^{a} f\right\|_{L^{2}(S)} \lesssim\left\|\Lambda^{b} f\right\|_{L^{2}(S)}
$$

for all $a \leq b$. We shall also make use of the following nonsharp Sobolev inequality:

Lemma 4.14. The following inequality holds for an arbitrary tensorfield $F$ on a surface $S=S_{s}, 0 \leq s \leq 1$, with $2<p<\infty, s>1-\frac{2}{p}$ :

$$
\|F\|_{L^{p}(S)} \lesssim\left\|\Lambda^{s} F\right\|_{L^{2}(S)}
$$

Proof : The proof is given in [Kl-Rodn3]. It only depends on the weak regularity assumption mentioned above and not on any bounds for $K$.

We denote by $K_{a}, \frac{1}{2}<a<1$, the constant we intend to bound,

$$
K_{a}=\left\|\Lambda^{-a}\left(K-\frac{1}{r^{2}}\right)\right\|_{L_{t}^{\infty} L_{x}^{2}}
$$

Recall that, see the derivation of (146) and the definition of $\check{\rho}$,

Clearly, on any $S=S_{s}$,

$$
K-\frac{1}{r^{2}}=\frac{1}{2 r} \underline{A}+A \cdot \underline{A}+\check{\rho}
$$

$$
\left\|\Lambda^{-a}\left(\frac{1}{2 r} \underline{A}\right)\right\|_{L^{2}(S)}=\frac{1}{2 r}\left\|\Lambda^{-a} \underline{A}_{L^{2}(S)} \lesssim\right\| \underline{A}\left\|_{L^{2}(S)} \lesssim\right\| \underline{A} \|_{L_{t}^{\infty} L_{x}^{2}} \lesssim \Delta_{0}
$$

On the other hand, to estimate the norm $J_{a}(s)=\left\|\Lambda^{-a}(A \cdot \underline{A})\right\|_{L^{2}\left(S_{s}\right)}, 0 \leq s \leq 1$ we proceed as follows,

$$
\begin{aligned}
J_{a}^{2}(s) & =\int_{s} \Lambda^{-2 a}(A \cdot \underline{A}) \cdot(A \cdot \underline{A}) \lesssim\left\|\Lambda^{-2 a}(A \cdot \underline{A}) \cdot A\right\|_{L^{2}\left(S_{s}\right)} \cdot\|\underline{A}\|_{L^{2}\left(S_{s}\right)} \\
& \lesssim \Delta_{0} \cdot\left\|\left.\Lambda^{-2 a}(A \cdot \underline{A})\right|_{L^{4}\left(S_{s}\right)} \cdot\right\| A \|_{L^{4}\left(S_{s}\right)}
\end{aligned}
$$

or, in view of (143),

$$
J_{a}^{2}(s) \lesssim \Delta_{0}^{2} \cdot\left\|\Lambda^{-2 a}(A \cdot \underline{A})\right\|_{L^{4}\left(S_{s}\right)}
$$

Applying the non-sharp Sobolev inequality (149) with $p=4$ and $\frac{1}{2}<\delta \leq a$,

$$
J_{a}^{2}(s) \lesssim \Delta_{0}^{2} \cdot\left\|\Lambda^{-2 a+\delta}(A \cdot \underline{A})\right\|_{L^{2}\left(S_{s}\right)} \lesssim \Delta_{0}^{2} \cdot\left\|\Lambda^{-a}(A \cdot \underline{A})\right\|_{L^{2}\left(S_{s}\right)}=\Delta_{0}^{2} \cdot J_{a}
$$

and therefore, since $\Delta_{0}$ is small, $J_{a} \lesssim \Delta_{0}^{2}$. Therefore,

$$
\left\|\Lambda^{-a}(A \cdot \underline{A})\right\|_{L_{t}^{\infty} L_{x}^{2}} \lesssim \Delta_{0}^{2}
$$


So far we have established the following estimate,

$$
\left\|\Lambda^{-a}\left(K-\frac{1}{r^{2}}-\check{\rho}\right)\right\|_{L_{t}^{\infty} L_{x}^{2}} \lesssim \Delta_{0}
$$

It remains to estimate the more diffficult curvature term $\left\|\Lambda^{-a} \check{\rho}\right\|_{L_{t}^{\infty} L_{x}^{2}}$. To tackle it we need to integrate in $t$ and bring in a $\nabla_{L}$ derivative. Set

$$
W(s)=\left\|\Lambda^{-a} \check{\rho}\right\|_{L^{2}\left(S_{s}\right)}^{2}-\left\|\Lambda^{-a} \check{\rho}\right\|_{L^{2}\left(S_{0}\right)}^{2} .
$$

and proceed as follows:

$$
\begin{aligned}
W(s) & =\int_{S} \Lambda^{-a} \check{\rho} \cdot \Lambda^{-a} \check{\rho}-\int_{S_{0}} \Lambda^{-a} \check{\rho} \cdot \Lambda^{-a} \check{\rho} \\
& \approx \int_{S_{0}} \Lambda^{-a} \check{\rho} \cdot \Lambda^{-a} \check{\rho}(s, \omega)-\Lambda^{-a} \check{\rho} \cdot \Lambda^{-a} \check{\rho}(s, \omega) \\
& =\int_{S_{0}} \int_{0}^{s} \nabla_{L}\left(\Lambda^{-a} \check{\rho} \cdot \Lambda^{-a} \check{\rho}(s, \omega)\right) d s \\
& =2 \int_{S_{0}} \int_{0}^{s}\left(\nabla_{L}\left(\Lambda^{-a} \check{\rho}\right) \cdot \Lambda^{-a} \check{\rho}(s, \omega)\right. \\
& =\int_{S_{0}} \int_{0}^{s} \Lambda^{-2 a}\left(\nabla_{L} \check{\rho}(s, \omega)\right) \cdot \check{\rho}+\int_{S_{0}} \int_{0}^{s}\left[\nabla_{L}, \Lambda^{-a}\right] \check{\rho} \cdot \Lambda^{-a} \check{\rho} \\
& \lesssim\left\|\Lambda^{-2 a}\left(\nabla_{L} \check{\rho}\right)\right\|_{L_{t}^{2} L_{x}^{2}} \cdot\|\check{\rho}\|_{L_{t}^{2} L_{x}^{2}}+\left\|\left[\nabla_{L}, \Lambda^{-a}\right] \check{\rho}\right\|_{L_{t}^{1} L_{x}^{2}} \cdot\left\|\Lambda^{-a} \check{\rho}\right\|_{L_{t}^{\infty} L_{x}^{2}}
\end{aligned}
$$

We can also estimate $\left\|\Lambda^{-a} \check{\rho}\right\|_{L^{2}\left(S_{0}\right)}^{2}$ in the same manner using a cut-off function as in the proof of (138). Thus,

$\left\|\Lambda^{-a} \check{\rho}\right\|_{L_{t}^{\infty} L_{x}^{2}}^{2} \lesssim\left\|\Lambda^{-2 a}\left(\nabla_{L} \check{\rho}\right)\right\|_{L_{t}^{2} L_{x}^{2}} \cdot\|\check{\rho}\|_{L_{t}^{2} L_{x}^{2}}+\left\|\left[\nabla_{L}, \Lambda^{-a}\right] \check{\rho}\right\|_{L_{t}^{1} L_{x}^{2}} \cdot\left\|\Lambda^{-a} \check{\rho}\right\|_{L_{t}^{\infty} L_{x}^{2}}$

and therefore,

$$
\left\|\Lambda^{-a} \check{\rho}\right\|_{L_{t}^{\infty} L_{x}^{2}} \lesssim\left\|\Lambda^{-2 a}\left(\nabla_{L} \check{\rho}\right)\right\|_{L_{t}^{2} L_{x}^{2}}+\|\check{\rho}\|_{L_{t}^{2} L_{x}^{2}}+\left\|\left[\nabla_{L}, \Lambda^{-a}\right] \check{\rho}\right\|_{L_{t}^{1} L_{x}^{2}}
$$

To estimate the commutator term in (153) we need the following:

Lemma 4.15. The following estimate holds true for scalar functions $f$ on $\mathcal{H}$ with some snall $\epsilon>0$ :

$$
\left\|\left[\nabla_{L}, \Lambda^{-a}\right] f\right\|_{L_{t}^{1} L_{x}^{2}} \lesssim I_{a}^{\epsilon} \cdot\|f\|_{L_{t}^{2} L_{x}^{2}}
$$

with $I_{a}=1+K_{a}^{\frac{1}{1-a}}+K_{a}^{\frac{1}{2}}$

Proof : We postpone the proof, which requires some properties of the heat flow defining the fractional operators $\Lambda^{a}$, to the appendix.

In view of the lemma we infer that,

$$
\left\|\Lambda^{-a} \check{\rho}\right\|_{L_{t}^{\infty} L_{x}^{2}} \lesssim\left\|\Lambda^{-2 a}\left(\nabla_{L} \check{\rho}\right)\right\|_{L_{t}^{2} L_{x}^{2}}+\left(1+I_{a}^{\epsilon}\right) \cdot\|\check{\rho}\|_{L_{t}^{2} L_{x}^{2}}
$$


To estimate $\left\|\Lambda^{-2 a} \nabla_{L} \check{\rho}\right\|_{L_{t}^{2} L_{x}^{2}}$ we need to use the renormalized ${ }^{28}$ null Bianchi (72), which we write in the following symbolic form

$$
\begin{aligned}
L(\check{\rho})+\frac{3}{2} \operatorname{tr} \chi \cdot \check{\rho} & =\operatorname{div} \beta-\zeta \cdot \beta+\frac{1}{2} \hat{\chi} \cdot\left(\nabla \widehat{\otimes} \zeta+\frac{1}{2} \operatorname{tr} \underline{\chi} \cdot \hat{\chi}-\zeta \widehat{\otimes} \zeta\right) \\
& =\operatorname{div} \beta+A \cdot(R+\nabla A+A \cdot A)+\frac{1}{2}\left(\operatorname{tr} \underline{\chi}+\frac{2}{r}\right) \cdot|\hat{\chi}|^{2}+\frac{1}{r} A \cdot A \\
& =\operatorname{div} \beta+A \cdot\left(R+\frac{1}{r} A+\nabla A+A \cdot \underline{A}\right)
\end{aligned}
$$

Denote $\bar{R}=\left(R+\frac{1}{r} A+\nabla A+A \cdot \underline{A}\right)$ and observe that, in view of BA1-BA3, it satisfies the estimate,

$$
\|\bar{R}\|_{L_{t}^{2} L_{x}^{2}} \lesssim \mathcal{R}_{0}+\Delta_{0}+\|A \cdot \underline{A}\|_{L_{t}^{2} L_{x}^{2}} \lesssim \Delta_{0}
$$

Therefore,

$$
\left\|\Lambda^{-2 a} \nabla_{L} \rho\right\|_{L_{t}^{2} L_{x}^{2}} \lesssim\left\|\Lambda^{-2 a} \operatorname{div} \beta\right\|_{L_{t}^{2} L_{x}^{2}}+\left\|\Lambda^{-2 a}(A \cdot \bar{R})\right\|_{L_{t}^{2} L_{x}^{2}}
$$

To show that $R_{a}=\left\|\Lambda^{-2 a}(A \cdot \bar{R})\right\|_{L_{t}^{2} L_{x}^{2}} \lesssim \Delta_{0}^{2}$ we use the same trick as we have used above for $J_{a}$.

$$
\begin{aligned}
R_{a}^{2} & =\int_{\mathcal{H}} \Lambda^{-4 a}(A \cdot \bar{R}) \cdot(A \cdot \bar{R}) \lesssim\left\|\Lambda^{-4 a}(A \cdot \bar{R})\right\|_{L_{t}^{2} L_{x}^{4}} \cdot\|A\|_{L_{t}^{\infty} L_{x}^{4}} \cdot\|\bar{R}\|_{L_{t}^{2} L_{x}^{2}} \\
& \lesssim \Delta_{0}^{2} \cdot\left\|\Lambda^{-4 a+\delta}(A \cdot \bar{R})\right\|_{L_{t}^{2} L_{x}^{2}} \lesssim \Delta_{0}^{2} \cdot\left\|\Lambda^{-2 a}(A \cdot \bar{R})\right\|_{L_{t}^{2} L_{x}^{2}}=\Delta_{0}^{2} \cdot R_{a}
\end{aligned}
$$

Thus, recalling (155),

$$
\begin{aligned}
\left\|\Lambda^{-a} \check{\rho}\right\|_{L_{t}^{\infty} L_{x}^{2}} & \lesssim\left\|\Lambda^{-2 a} \nabla_{L} \check{\rho}\right\|_{L_{t}^{2} L_{x}^{2}}+\left(1+I_{a}^{\epsilon}\right) \cdot\|\check{\rho}\|_{L_{t}^{2} L_{x}^{2}} \\
& \lesssim\left\|\Lambda^{-2 a} \operatorname{div} \beta\right\|_{L_{t}^{2} L_{x}^{2}}+\left(1+I_{a}^{\epsilon}\right) \cdot \Delta_{0}
\end{aligned}
$$

In the next lemma we shall show that $\left\|\Lambda^{-2 a} \operatorname{div} \beta\right\|_{L_{t}^{2} L_{x}^{2}} \lesssim \Delta_{0}$. Assuming it for a moment we derive,

$$
\left\|\Lambda^{-a} \check{\rho}\right\|_{L_{t}^{\infty} L_{x}^{2}} \lesssim\left(1+I_{a}^{\epsilon}\right) \cdot \Delta_{0}
$$

Thus, together with (152),

$$
\begin{aligned}
K_{a} & \lesssim\left\|\Lambda^{-a}\left(K-\frac{1}{r^{2}}-\check{\rho}\right)\right\|_{L_{t}^{\infty} L_{x}^{2}}+\left\|\Lambda^{-a} \check{\rho}\right\|_{L_{t}^{\infty} L_{x}^{2}} \lesssim\left(1+I_{a}^{\epsilon}\right) \cdot \Delta_{0} \\
& \lesssim \Delta_{0}+\Delta_{0} \cdot\left(1+K_{a}^{\frac{1}{1-a}}+K_{a}^{\frac{1}{2}}\right)^{\epsilon}
\end{aligned}
$$

Thus, for every $a, 1 / 2<a<1$, we can pick $\epsilon$ sufficiently small so that $\epsilon \cdot \frac{1}{1-a}<1$ and therefore $K_{a} \lesssim \Delta_{0}$ as desired. It only remains to prove the following:

Lemma 4.16. For a 1-form $F$ and $a \geq \frac{1}{2}$ we have

$$
\left\|\Lambda^{-2 a} \operatorname{div} F\right\|_{L_{t}^{2} L_{x}^{2}} \lesssim\|F\|_{L_{t}^{2} L_{x}^{2}}
$$

Proof: By duality it suffices to prove, for scalar functions $f$ on $\mathcal{H}$,

$$
\left\|\nabla \cdot \Lambda^{-2 a} f\right\|_{L_{t}^{2} L_{x}^{2}} \lesssim\|f\|_{L_{t}^{2} L_{x}^{2}}
$$

\footnotetext{
${ }^{28}$ Note that the standard Bianchi identity 41 for $\rho$ would lead to dificculties because of the presence of terms like $\underline{A} \cdot R$.
} 
Now, on each $S=S_{s}$, since $\Delta$ commutes with $\Lambda^{-2 a}$,

$$
\begin{aligned}
\left\|\nabla \Lambda^{-2 a} f\right\|_{L^{2}(S)}^{2} & =\int_{S} \Delta \Lambda^{-2 a} f \cdot \Lambda^{-2 a} f=\int_{S} \Delta \Lambda^{-4 a} f \cdot f \\
& \lesssim\left\|\Delta \Lambda^{-4 a} f\right\|_{L^{2}(S)} \cdot\|f\|_{L^{2}(S)} \lesssim\|f\|_{L^{2}(S)}^{2}
\end{aligned}
$$

This ends the proof of proposition 4.13.

The bound on $\left\|\Lambda^{-a}\left(K-\frac{1}{r^{2}}\right)\right\|_{L_{t}^{2} L_{x}^{2}}$ allows us to prove the following elliptic estimates:

Proposition 4.17. Under the bootstrap assumptions BA1-BA3 we infer that,

$$
\left\|\nabla^{2} f\right\|_{L^{2}(S)}+\|\nabla f\|_{L^{2}(S)} \lesssim\|\Delta f\|_{L^{2}(S)}
$$

Thus, in particular

$$
\left\|\nabla^{2}\left(\Delta^{-1} f\right)\right\|_{L^{2}(S)}+\left\|\nabla^{2}\left(\Delta^{-1} f\right)\right\|_{L^{2}(S)} \lesssim\|f\|_{L^{2}(S)}
$$

Proof The proof is based on the following,

Proposition 4.18. The following Bochner identity holds true on any 2-surface $S=S_{s}$ and scalar function $f$,

$$
\int_{S}\left|\nabla^{2} f\right|^{2}=\int_{S}|\Delta f|^{2}-\int_{S} K|\nabla f|^{2}
$$

Proof : Recall that on a 2-surface the Riemann tensor $R_{a b c d}=\left(\gamma_{a c} \gamma_{b d}-\gamma_{a d} \gamma_{b c}\right) K$ In particular, $R_{a b}=\gamma_{a b} K$. We start with the identity

$$
\left|\nabla^{2} f\right|^{2}=f_{; a b} f^{; a b}=f_{; a b} f^{; b a}=\nabla^{a}\left(f_{; a b} \nabla^{b} f\right)-\nabla^{a} f_{; a b} \nabla^{b} f
$$

Furthermore,

$$
\nabla^{a} f_{; a b} \nabla^{b} f=\nabla_{b} \Delta f \nabla^{b} f+R_{a b} \nabla^{a} f \nabla^{b} f=\nabla_{b}\left(\Delta f \nabla^{b} f\right)-|\Delta f|^{2}+K|\nabla f|^{2}
$$

Integrating over $S$ and using the Stokes' formula we obtain (161).

We now write $K=\left(K-\frac{1}{r^{2}}\right)+\frac{1}{r^{2}}$ in (161), and estimate with the help of proposition 4.13 ,

$$
\begin{aligned}
\int_{S}\left|\nabla^{2} f\right|^{2}+\frac{1}{r^{2}} \int_{S}|\nabla f|^{2} & =\int_{S}|\Delta f|^{2}-\int_{S}\left(K-\frac{1}{r^{2}}\right)|\nabla f|^{2} \\
& =\int_{S}|\Delta f|^{2}-\int_{S} \Lambda^{-a}\left(K-\frac{1}{r^{2}}\right) \cdot \Lambda^{a}|\nabla f|^{2} \\
& \lesssim \int_{S}|\Delta f|^{2}+\Delta_{0} \cdot\left\|\Lambda^{a}|\nabla f|^{2}\right\|_{L^{2}(S)}
\end{aligned}
$$

To estimate the term $\left\|\Lambda^{a}|\nabla f|^{2}\right\|_{L^{2}(S)}$ we need the following product estimates, 
Proposition 4.19. The following product estimate holds true for arbitrary tensors $F, G$ on $S$,

$$
\left\|\Lambda^{a}(F \cdot G)\right\|_{L^{2}(S)} \lesssim\|\Lambda F\|_{L^{2}(S)} \cdot\left\|\Lambda^{a} G\right\|_{L^{2}(S)}+\left\|\Lambda^{a} F\right\|_{L^{2}(S)} \cdot\|\Lambda G\|_{L^{2}(S)}
$$

Proof : The proof, based on geometric LP theory, is given in [Kl-Rodn3]. It is interesting to note that these product estimates do not depend on the quantity $K_{a}=\left\|\Lambda^{-a}\left(K-\frac{1}{r^{2}}\right)\right\|_{L_{t}^{\infty} L_{x}^{2}}$.

In view of proposition 4.19 we derive:

$$
\begin{aligned}
\left\|\Lambda^{a}|\nabla f|^{2}\right\|_{L^{2}(S)} & \lesssim\|\Lambda \nabla f\|_{L^{2}(S)}\left\|\Lambda^{a} \nabla f\right\|_{L^{2}(S)} \lesssim\|\Lambda \nabla f\|_{L^{2}(S)}^{2} \\
& \lesssim\left(\left\|\nabla^{2} f\right\|_{L^{2}(S)}+\|\nabla f\|_{L^{2}(S)}\right)^{2}
\end{aligned}
$$

Thus, back to (162), we infer in view of $r \geq 1$ (see(119)),

$$
\left\|\nabla^{2} f\right\|_{L^{2}(S)}+\|\nabla f\|_{L^{2}(S)} \lesssim\|\Delta f\|_{L^{2}(S)}
$$

This ends the proof of proposition 4.17.

4.20. $L^{2}$ Estimates for Hodge systems. We recall the following identities for Hodge systems:

Proposition 4.21. Let $(S, \gamma)$ be a compact manifold with Gauss curvature K.

i.) The following identity holds for vectorfields $F$ on $S$ :

$$
\int_{S}\left(|\nabla F|^{2}+K|F|^{2}\right)=\int_{S}\left(|\operatorname{div} F|^{2}+|\operatorname{curl} F|^{2}\right)=\int_{S}\left|\mathcal{D}_{1} F\right|^{2}
$$

ii.) The following identity holds for symmetric, traceless, 2-tensorfields $F$ on $S$ :

$$
\int_{S}\left(|\nabla F|^{2}+2 K|F|^{2}\right)=2 \int_{S}|\operatorname{div} F|^{2}=2 \int_{S}\left|\mathcal{D}_{2} F\right|^{2}
$$

iii.) The following identity holds for pairs of functions $(\rho, \sigma)$ on $S$ :

$$
\int_{S}\left(|\nabla \rho|^{2}+|\nabla \sigma|^{2}\right)=\int_{S}\left|-\nabla \rho+(\nabla \sigma)^{\star}\right|^{2}=\int_{S}\left|{ }^{\star} \mathcal{D}_{1}(\rho, \sigma)\right|^{2}
$$

iv.) The following identity holds for vectors $F$ on $S$,

$$
\int_{S}\left(|\nabla F|^{2}-K|F|^{2}\right)=2 \int_{S}\left|{ }^{\star} \mathcal{D}_{2} F\right|^{2}
$$

Proof : All the above identities follow easily from the formulas (92) and (93). Indeed let $\mathcal{D}$ be any of the operators $\mathcal{D}_{1}, \mathcal{D}_{2},{ }^{\star} \mathcal{D}_{1},{ }^{\star} \mathcal{D}_{2}$. Then, for a corresponding tensor $U$,

$$
\|\mathcal{D} U\|_{L^{2}(S)}^{2}=<^{*} \mathcal{D} \cdot \mathcal{D} U, U>.
$$


We give below a direct derivation of the identities (166) and (167), the only ones which were not not dicussed in [Chr-Kl]. Observe that $\left|-\nabla \rho+(\nabla \sigma)^{\star}\right|^{2}=|\nabla \rho|^{2}+$ $|\nabla \sigma|^{2}-2 \nabla \rho \cdot(\nabla \sigma)^{\star}$ and that, integrating by parts,

$$
\int_{S} \nabla \rho \cdot(\nabla \sigma)^{\star}=\int_{S} \epsilon^{a b} \nabla_{a} \rho \nabla_{b} \sigma=\int_{S} \epsilon^{a b}\left(\nabla_{b} \nabla_{a} \rho\right) \sigma=0 .
$$

Recall that $\left({ }^{\star} \mathcal{D}_{2} F\right)_{a b}=-\frac{1}{2}\left(\nabla_{b} F_{a}+\nabla_{a} F_{b}-\operatorname{div} F \gamma_{a b}\right)$. Therefore, relative to an arbitrary ortonormal frame on $S$,

$$
\left.\left.4\right|^{\star} \mathcal{D}_{2} F\right|^{2}=2|\nabla F|^{2}+2|\operatorname{div} F|^{2}+2 \sum_{a, b=1,2} \nabla_{b} F_{a} \nabla_{a} F_{b}-4|\operatorname{div} F|^{2}
$$

and therefore,

$$
\begin{aligned}
4 \int_{S}\left|{ }^{\star} \mathcal{D}_{2} F\right|^{2} & =2 \int_{S}|\nabla F|^{2}-2 \int_{S}|\operatorname{div} F|^{2}+2 \sum_{a, b=1,2} \int_{S} \nabla_{b} F_{a} \cdot \nabla_{a} F_{b} \\
& =2 \int_{S}|\nabla F|^{2}-2 \sum_{a, b=1,2} \int_{S}\left(\nabla_{a} \nabla_{b}-\nabla_{b} \nabla_{a}\right) F_{a} \cdot F_{b} \\
& =2 \int_{S}\left(|\nabla F|^{2}-K|F|^{2}\right)
\end{aligned}
$$

as desired.

In view of (147) we rewrite (164), on each $S=S_{s} \subset \mathcal{H}$, in the form,

$$
\begin{aligned}
\int_{S}\left(|\nabla F|^{2}+\frac{1}{r^{2}}|F|^{2}\right) & =2 \int_{S}\left(|\operatorname{div} F|^{2}+|\operatorname{curl} F|^{2}\right)-\int_{S}\left(K-\frac{1}{r^{2}}\right)|F|^{2} \\
& =\int_{S}\left(|\operatorname{div} F|^{2}+|\operatorname{curl} F|^{2}\right)-\int_{S} \Lambda^{-a}\left(K-\frac{1}{r^{2}}\right) \Lambda^{a}|F|^{2} \\
& \lesssim\left\|\mathcal{D}_{1} F\right\|_{L^{2}(S)}^{2}+\left\|\Lambda^{-a}\left(K-\frac{1}{r^{2}}\right)\right\|_{L^{2}(S)} \cdot\left\|\Lambda^{a}|F|^{2}\right\|_{L^{2}(S)}
\end{aligned}
$$

for some $\frac{1}{2}<a<1$ sufficiently close to $\frac{1}{2}$. Thus, in view of the estimate(148) of proposition 4.13 and the product estimates of proposition 4.19 ,

$$
\begin{aligned}
\int_{S}\left(|\nabla F|^{2}+\frac{1}{r^{2}}|F|^{2}\right) & \lesssim\left\|\mathcal{D}_{1} F\right\|_{L^{2}(S)}^{2}+\Delta_{0} \cdot\|\Lambda F\|_{L^{2}(S)}\left\|\Lambda^{a} F\right\|_{L^{2}} \\
& \lesssim\left\|\mathcal{D}_{1} F\right\|_{L^{2}(S)}^{2}+\Delta_{0} \cdot\|\Lambda F\|_{L^{2}(S)}^{2} \\
& \lesssim\left\|\mathcal{D}_{1} F\right\|_{L^{2}(S)}^{2}+\Delta_{0}\left(\|F\|_{L^{2}(S)}^{2}+\|\nabla F\|_{L^{2}}^{2}\right)
\end{aligned}
$$

Since $r \leq r_{0}+\frac{3 s}{2} \leq 3 r_{0} \lesssim 1$ we deduce that,

$$
\|F\|_{L^{2}(S)}^{2}+\|\nabla F\|_{L^{2}(S)}^{2} \lesssim\left\|\mathcal{D}_{1} F\right\|_{L^{2}(S)}^{2}+\Delta_{0}\left(\|F\|_{L^{2}(S)}^{2}+\|\nabla F\|_{L^{2}}^{2}\right)
$$

and therefore, for sufficiently small $\Delta_{0}$,

$$
\|F\|_{L^{2}(S)}^{2}+\|\nabla F\|_{L^{2}}^{2} \lesssim\left\|\mathcal{D}_{1} F\right\|_{L^{2}(S)}^{2}
$$

Proceeding in the same manner, from the Hodge identity (165), and recalling the definition of $\mathcal{D}_{2}$ we deduce, for traceless, symmetric 2-tensors $F$,

$$
\|F\|_{L^{2}(S)}^{2}+\|\nabla F\|_{L^{2}}^{2} \lesssim\left\|\mathcal{D}_{2} F\right\|_{L^{2}(S)}^{2}
$$


On the other hand, recalling the definition of ${ }^{\star} \mathcal{D}_{1}$ we deduce from $(166)$,

$$
\|\nabla \rho\|_{L^{2}(S)}^{2}+\|\nabla \sigma\|_{L^{2}(S)}^{2}=\int_{\mathcal{H}}\left|{ }^{\star} \mathcal{D}_{1}(\rho, \sigma)\right|^{2}
$$

We summarize these results in the following

Proposition 4.22. The following estimates hold on an arbitrary 2-surface $S=$ $S_{s} \subset \mathcal{H}:$

i.) The operator $\mathcal{D}_{1}$ is invertible on its range and its inverse $\mathcal{D}_{1}^{-1}$ takes pair of functions $f=(\rho, \sigma)$ (in the range of $\mathcal{D}_{1}$ ) into $S$-tangent 1 -forms $F$ with div $F=$ $\rho$, curl $F=\sigma$ with estimate,

$$
\left\|\nabla \cdot \mathcal{D}_{1}^{-1} F\right\|_{L^{2}(S)}+\left\|\mathcal{D}_{1}^{-1} F\right\|_{L^{2}(S)} \lesssim\|F\|_{L^{2}(S)}
$$

ii.) The operator $\mathcal{D}_{2}$ is invertible on its range and its inverse $\mathcal{D}_{2}^{-1}$ takes $S$ tangent 1 -forms $F$ (in the range of $\mathcal{D}_{2}$ ) into $S$-tangent symmetric, traceless, 2-tensorfields $Z$ with div $Z=F$ with estimate,

$$
\left\|\nabla \cdot \mathcal{D}_{2}^{-1} F\right\|_{L^{2}(S)}+\left\|\mathcal{D}_{2}^{-1} F\right\|_{L^{2}(S)} \lesssim\|F\|_{L^{2}(S)}
$$

iii.) The operator $(-\Delta)$ is invertible on its range $e^{29}$ and its inverse $(-\Delta)^{-1}$ verifies the estimate

$$
\left\|\nabla^{2}(-\Delta)^{-1} f\right\|_{L^{2}(S)}+\left\|\nabla(-\Delta)^{-1} f\right\|_{L^{2}(S)} \lesssim\|f\|_{L^{2}(S)}
$$

iv.) The operator ${ }^{\star} \mathcal{D}_{1}$ is invertible as an operator defined from pairs of $H^{1}$ functions with mean zero(i.e. the quotient of $H^{1}$ by the kernel of ${ }^{\star} \mathcal{D}_{1}$ ) and its inverse ${ }^{\star} \mathcal{D}_{1}^{-1}$ takes $S$-tangent $L^{2} 1$-forms $F$ (i.e. the full range of ${ }^{\star} \mathcal{D}_{1}$ ) into pair of functions $(\rho, \sigma)$ with mean zero, such that $-\nabla \rho+(\nabla \sigma)^{\star}=F$, with estimate,

$$
\left\|\nabla \cdot{ }^{\star} \mathcal{D}_{1}^{-1} F\right\|_{L^{2}(S)} \lesssim\|F\|_{L^{2}(S)}
$$

v.) The operator ${ }^{\star} \mathcal{D}_{2}$ is invertible as an operator defined on the quotient of $H^{1}$ vectorfields by the kernel of ${ }^{\star} \mathcal{D}_{2}$. Its inverse ${ }^{\star} \mathcal{D}_{2}^{-1}$ takes $S$-tangent, $L^{2}$, 2-forms $Z$ (i.e. the full range of ${ }^{\star} \mathcal{D}_{2}$ ) into $S$ tangent 1-forms $F$ ( orthogonal to the kernel of $\left.\mathcal{D}_{2}\right)$, such that ${ }^{\star} \mathcal{D}_{2} F=Z$, with estimate,

$$
\left\|\nabla \cdot{ }^{\star} \mathcal{D}_{2}^{-1} Z\right\|_{L^{2}(S)} \lesssim\|Z\|_{L^{2}(S)}
$$

Proof: It remains to $\left.\operatorname{prove}^{30} \mathbf{v}\right)$. The complication in this case is that the curvature term on the left hand side of (167) has a negative sign. This correponds to the nontriviality of the kernel of ${ }^{\star} \mathcal{D}_{2}$. The proof of (172) requires a contradiction argument. As we will not need the estimate in this paper we omit the argument here.

\footnotetext{
${ }^{29}$ Which consists of functions of mean zero.

${ }^{30}$ We have already proved iii in proposition 4.17 .
} 
We consider now commutators between $L$ and $\mathcal{D}_{1}^{-1}, \mathcal{D}_{2}^{-1}$, ${ }^{\star} \mathcal{D}_{1}^{-1}$. Consider first

$$
\left[L, \mathcal{D}_{1}^{-1}\right](\rho, \sigma):=\nabla_{L} \mathcal{D}_{1}^{-1}(\rho, \sigma)-\mathcal{D}_{1}^{-1}(L \rho, L \sigma) .
$$

Let $\hat{F}=\mathcal{D}_{1}^{-1}(\rho, \sigma)$ and $F=\mathcal{D}_{1}^{-1}(L \rho, L \sigma)$, i.e.

$$
\begin{aligned}
L(\operatorname{div} \hat{F}) & =L \rho, & L(\operatorname{curl} \hat{F}) & =L \sigma \\
\operatorname{div} F & =L \rho, & & \operatorname{curl} F=L \sigma
\end{aligned}
$$

Therefore,

$$
\begin{aligned}
\operatorname{div}\left(\nabla_{L} \hat{F}-F\right) & =[\operatorname{div}, L] \hat{F} \\
\operatorname{curl}\left(\nabla_{L} \hat{F}-F\right) & =[\operatorname{curl}, L] \hat{F}
\end{aligned}
$$

whence,

$\nabla_{L} \hat{F}-F=\mathcal{D}_{1}^{-1}([\operatorname{div}, L] \hat{F},[\operatorname{curl}, L] \hat{F})=\mathcal{D}_{1}^{-1}\left(\left[\mathcal{D}_{1}, L\right] \hat{F}\right)=\mathcal{D}_{1}^{-1}\left[\mathcal{D}_{1}, L\right] \mathcal{D}_{1}^{-1}(\rho, \sigma)$

or,

$$
\left[L, \mathcal{D}_{1}^{-1}\right]=\mathcal{D}_{1}^{-1}\left[\mathcal{D}_{1}, L\right] \mathcal{D}_{1}^{-1}
$$

Proceeding in precisely same manner for the other Hodge operators we infer that,

Lemma 4.23. Let $\mathcal{D}^{-1}$ be any of the operators $\mathcal{D}_{1}^{-1}, \mathcal{D}_{2}^{-1},{ }^{\star} \mathcal{D}_{1}^{-1}$. Then,

$$
\left[L, \mathcal{D}^{-1}\right]=\mathcal{D}^{-1}[\mathcal{D}, L] \mathcal{D}^{-1}
$$

We shall also need second derivative estimates. We start with $\mathcal{D}_{1} F=(\rho, \sigma)$. In view of $(92)$,

$$
(-\Delta+K) F={ }^{\star} \mathcal{D}_{1}\left(\mathcal{D}_{1} F\right)={ }^{\star} \mathcal{D}_{1}(\rho, \sigma) .
$$

We now recall the Bochner identity for 1-forms $F$ on $S=S_{s}$,

$$
\int_{S}\left|\nabla^{2} F\right|^{2}=\int_{S}|\Delta F|^{2}-\int_{S} K\left(2|\nabla F|^{2}-|\operatorname{div} F|^{2}\right)+\int_{S} K^{2}|F|^{2}
$$

Therefore, proceeding as for the first derivative estimates, since $\left\|\Lambda^{-a}\left(K-\frac{1}{r^{2}}\right)\right\|_{L^{2}(S)} \lesssim$ $\Delta_{0}$, and with the help of the product estimates of proposition 4.19 ,

$$
\begin{aligned}
\left|\int_{S}\left(K-\frac{1}{r^{2}}\right)\left(2|\nabla F|^{2}-|\operatorname{div} F|^{2}\right)\right| & \lesssim \Delta_{0} \cdot\left\|\Lambda^{a}\left(2|\nabla F|^{2}-|\operatorname{div} F|^{2}\right)\right\|_{L^{2}(S)} \\
& \lesssim \Delta_{0}\left(\left\|\nabla^{2} F\right\|_{L^{2}(S)}^{2}+\|\nabla F\|_{L^{2}(S)}^{2}\right)
\end{aligned}
$$

we derive,

$$
\begin{aligned}
\left\|\nabla^{2} F\right\|_{L^{2}(S)}^{2}+\|\nabla F\|_{L^{2}(S)} & \lesssim\left\|{ }^{\star} \mathcal{D}_{1}(\rho, \sigma)\right\|_{L^{2}(S)}^{2}+\Delta_{0}\left(\left\|\nabla^{2} F\right\|_{L^{2}(S)}^{2}+\|\nabla F\|_{L^{2}(S)}^{2}\right) \\
& +\|F\|_{L^{\infty}(S)}^{2}\|K\|_{L^{2}(S)}^{2}
\end{aligned}
$$

and therefore, for sufficiently small $\Delta_{0}$,

$$
\left\|\nabla^{2} F\right\|_{L^{2}(S)}^{2}+\|\nabla F\|_{L^{2}(S)} \lesssim\left\|{ }^{\star} \mathcal{D}_{1}(\rho, \sigma)\right\|_{L^{2}(S)}^{2}+\|F\|_{L^{\infty}(S)}^{2}\|K\|_{L^{2}(S)}^{2}
$$

Thus integrating over $s$,

$$
\begin{aligned}
\left\|\nabla^{2} F\right\|_{L_{t}^{2} L_{x}^{2}}^{2}+\|\nabla F\|_{L_{t}^{2} L_{x}^{2}}^{2} & \lesssim\left\|{ }^{\star} \mathcal{D}_{1}(\rho, \sigma)\right\|_{L_{t}^{2} L_{x}^{2}}^{2}+\|F\|_{L^{\infty}}^{2}\|K\|_{L_{t}^{2} L_{x}^{2}}^{2} \\
& \lesssim\left\|{ }^{\star} \mathcal{D}_{1}(\rho, \sigma)\right\|_{L_{t}^{2} L_{x}^{2}}^{2}+\Delta_{0}^{2}\|F\|_{L^{\infty}}^{2}
\end{aligned}
$$


Taking into account the estimate, see (141),

$$
\|F\|_{L^{\infty}}^{2} \lesssim \mathcal{N}_{2}(F)^{2} \lesssim\|F\|_{L_{t}^{2} L_{x}^{2}}^{2}+\left\|\nabla^{2} F\right\|_{L_{t}^{2} L_{x}^{2}}^{2}+\left\|\nabla_{L} F\right\|_{L_{t}^{2} L_{x}^{2}}^{2}+\left\|\nabla \nabla_{L} F\right\|_{L_{t}^{2} L_{x}^{2}}^{2}
$$

we infer that,

$$
\left.\left\|\nabla^{2} F\right\|_{L_{t}^{2} L_{x}^{2}}^{2}+\|\nabla F\|_{L_{t}^{2} L_{x}^{2}}^{2} \lesssim\left\|{ }^{\star} \mathcal{D}_{1}(\rho, \sigma)\right\|_{L_{t}^{2} L_{x}^{2}}^{2}+\Delta_{0}^{2}\left\|\nabla_{L} F\right\|_{L_{t}^{2} L_{x}^{2}}^{2}+\left\|\nabla \nabla_{L} F\right\|_{L_{t}^{2} L_{x}^{2}}^{2}\right)
$$

or, using the definition of $\mathcal{D}_{1}^{-1}$,

$$
\left\|\nabla^{2} \mathcal{D}_{1}^{-1}(\rho, \sigma) \lesssim\right\|{ }^{\star} \mathcal{D}_{1}(\rho, \sigma) \|_{L_{t}^{2} L_{x}^{2}}+\Delta_{0}\left(\left\|\nabla_{L} F\right\|_{L_{t}^{2} L_{x}^{2}}+\left\|\nabla \nabla_{L} F\right\|_{L_{t}^{2} L_{x}^{2}}\right)
$$

Similar estimates can be derived for $\mathcal{D}_{2}^{-1}$ and ${ }^{\star} \mathcal{D}_{1}^{-1}$.

Proposition 4.24. Let $\mathcal{D}$ denote either $\mathcal{D}_{1}$ or $\mathcal{D}_{2}$ with ${ }^{\star} \mathcal{D}$ and $\mathcal{D}^{-1}$ the corresponding adjoints and inverses. Then, for $F=(\rho, \sigma)$ pairs of scalar functions in the first case and $S$-tangent one forms for the second case, the following second order estimates hold true:

$$
\left\|\nabla^{2} \mathcal{D}^{-1} F\right\|_{L_{t}^{2} L_{x}^{2}} \lesssim\left\|{ }^{\star} \mathcal{D} F\right\|_{L_{t}^{2} L_{x}^{2}}+\Delta_{0}\left(\left\|\nabla_{L} \mathcal{D}^{-1} F\right\|_{L_{t}^{2} L_{x}^{2}}+\left\|\nabla \nabla_{L} \mathcal{D}^{-1} F\right\|_{L_{t}^{2} L_{x}^{2}}\right)
$$

We shall also need the following :

Proposition 4.25. Let $\mathcal{D}^{-1}$ denote either $\mathcal{D}_{1}^{-1}$ or $\mathcal{D}_{2}^{-1}$ Then, for $F=(\rho, \sigma)$ pairs of scalar functions in the first case and $S$-tangent one forms for the second case. For any $1<p \leq 2$,

$$
\left\|\mathcal{D}^{-1} F\right\|_{L^{2}(S)} \lesssim\|F\|_{L^{p}(S)}
$$

Proof : By duality it suffices to prove the estimate

$$
\left\|{ }^{\star} \mathcal{D}^{-1} F\right\|_{L^{q}(S)} \lesssim\|F\|_{L^{2}(S)}
$$

for $q^{-1}+p^{-1}=1$. In view of (149) of proposition 4.14,

$$
\begin{aligned}
\left\|{ }^{\star} \mathcal{D}^{-1} F\right\|_{L^{q}(S)} & \lesssim\left\|\Lambda^{\star} \mathcal{D}^{-1} F\right\|_{L^{2}(S)} \\
& \lesssim\left\|{ }^{\star} \mathcal{D}^{-1} F\right\|_{L^{2}(S)}+\left\|\nabla^{\star} \mathcal{D}^{-1} F\right\|_{L^{2}(S)} \lesssim\|F\|_{L^{2}(S)}
\end{aligned}
$$

in view of proposition 4.21

\section{Main Estimates in Besov Norms}

We present in this section our main lemmas concerning the Besov norms $\mathcal{B}^{0}$ and $\mathcal{P}^{0}$. These estimates, based on the properties of the LP projections $P_{k}$ and the bootstrap assumptions BA1 - BA2, are quite involved and require a separate paper for their proof, see [Kl-Rodn3], [Kl-Rodn4].

We start with two propositions concerning the Besov spaces $B_{2,1}^{\gamma}(S)$ which were proved in [Kl-Rodn3]. We remark that the estimates of [Kl-Rodn3] were proved based only on the assumption of weak regularity. This assumption holds for our surfaces $S_{s}$ in view of proposition 4.4. 
Proposition 5.1. For scalar functions $f$ on $S$ the following sharp Sobolev inequality holds,

$$
\|f\|_{L^{\infty}} \lesssim\|f\|_{B_{2,1}^{1}(S)}
$$

Morever,

$$
\|f\|_{B_{2,1}^{1}(S)} \lesssim\|f\|_{L^{2}(S)}+\|\nabla f\|_{B_{2,1}^{0}(S)}
$$

As a corollary we have for scalar functions on $\mathcal{H}$,

$$
\|f\|_{L_{t}^{\infty} L_{x}^{\infty}} \lesssim\|f\|_{\mathcal{B}^{1}} \lesssim\|f\|_{L_{t}^{\infty} L_{x}^{2}}+\|\nabla f\|_{\mathcal{B}^{0}}
$$

We shall need the following sharp version of product estimates.

Proposition 5.2. Let $f$ be a scalar and $U$ an arbitrary tensor on a fixed $S=S_{s}$. The following bilinear estimate holds true:

$$
\|f \cdot U\|_{B_{2,1}^{0}(S)} \lesssim\left(\|\nabla f\|_{L^{2}(S)}+\|f\|_{L^{\infty}(S)}\right)\|U\|_{B_{2,1}^{0}(S)}
$$

Also, on all $\mathcal{H}$,

$$
\|f \cdot U\|_{\mathcal{B}^{0}} \lesssim\left(\|\nabla f\|_{L_{t}^{\infty} L_{x}^{2}}+\|f\|_{L^{\infty}}\right)\|U\|_{\mathcal{B}^{0}}
$$

We shall need the following non sharp embedding estimate,

Proposition 5.3. Let $F$ be as $S$-tangent tensorfield on $\mathcal{H}$. The following non sharp embedding result holds for any $0 \leq \theta<\frac{1}{2}$ :

$$
\|F\|_{\mathcal{B}^{\theta}} \lesssim \mathcal{N}_{1}(F)
$$

As a consequence we infer the following estimate for $A$ :

$$
\|A\|_{\mathcal{B}^{\theta}} \lesssim \Delta_{0}
$$

Proof : See [Kl-Rodn4].

\subsection{Main Lemmas.}

Lemma 5.5 (Main Lemma). Consider the transport equation along $\mathcal{H}$ for an arbitrary $S$-tangent tensor $U$ :

$$
\nabla_{L} U=F
$$

with $F$ given tensor of same type. Assume $U(0)=0$ and that $F$ is one of the following types of bilinear expressions:

i. $\quad F=G \cdot \nabla_{L} P$. Then,

$$
\|U\|_{\mathcal{B}^{0}} \lesssim\left(\mathcal{N}_{1}(G)+\|G\|_{L_{x}^{\infty} L_{t}^{2}}\right) \cdot \mathcal{N}_{1}(P)
$$

ii. $F=G \cdot W$. Then,

$$
\|U\|_{\mathcal{B}^{0}} \lesssim\left(\mathcal{N}_{1}(G)+\|G\|_{L_{x}^{\infty} L_{t}^{2}}\right) \cdot\|W\|_{\mathcal{P}^{0}}
$$


iii. If $U(0) \neq 0$ and $F=0$,

$$
\|U\|_{\mathcal{B}^{0}} \lesssim\|U(0)\|_{B_{2,1}^{0}\left(S_{0}\right)}
$$

iv. If the tensorfield $W$ verifies the transport equation,

$$
\nabla_{L} W=E
$$

the following product estimate holds true,

$$
\|G \cdot W\|_{\mathcal{P}^{0}} \lesssim\left(\mathcal{N}_{1}(G)+\|G\|_{L_{x}^{\infty} L_{t}^{2}}\right) \cdot\left(\|W(0)\|_{B_{2,1}^{0}\left(S_{0}\right)}+\|E\|_{\mathcal{P}^{0}}\right)
$$

We shall also make use of the following nonsharp product estimates:

Proposition 5.6. For any $S$-tangent tensors $F, G$ we have, for $0 \leq \epsilon<1 / 2$,

$$
\begin{aligned}
\|F \cdot G\|_{\mathcal{P}^{\epsilon}} & \lesssim\left(\|F\|_{L_{t}^{2} L_{x}^{2}}+\|\nabla F\|_{L_{t}^{2} L_{x}^{2}}\right) \cdot\|G\|_{\mathcal{B}^{\epsilon}} \\
\|F \cdot G\|_{\mathcal{P}^{\epsilon}} & \lesssim \mathcal{N}_{2}(F) \cdot\|G\|_{\mathcal{P}^{\epsilon}} \\
\|F \cdot G\|_{\mathcal{P}^{\epsilon}} & \lesssim \mathcal{N}_{1}(F) \cdot\left(\|G\|_{L_{t}^{2} L_{x}^{2}}+\|\nabla G\|_{L_{t}^{2} L_{x}^{2}}\right)
\end{aligned}
$$

As a consequence of the main lemma we prove the following:

Proposition 5.7. Assume that the scalar function $U$ satisfies the transport equation along $\mathcal{H}$, for some positive number $k$.

$$
\frac{d}{d s} U+k t r \chi U=F_{1} \cdot \nabla_{L} P+F_{2} \cdot W .
$$

Then,

$$
\begin{aligned}
\|U\|_{\mathcal{B}^{0}} & \lesssim\|U(0)\|_{B_{2,1}^{0}\left(S_{0}\right)}+\left(\mathcal{N}_{1}\left(F_{1}\right)+\left\|F_{1}\right\|_{L_{x}^{\infty} L_{t}^{2}}\right) \cdot \mathcal{N}_{1}(P) \\
& +\left(\mathcal{N}_{1}\left(F_{2}\right)+\left\|F_{2}\right\|_{L_{x}^{\infty} L_{t}^{2}}\right) \cdot\|W\|_{\mathcal{P}^{0}}
\end{aligned}
$$

Remark 5.8. We shall see from the proof below that the precise form of the left hand side of the transport equations above does not matter; we can replace $k \operatorname{tr} \chi$ by any scalar function $\kappa$ such that $\int_{0}^{s} \kappa$ has the same properties of the integral factor $v_{s}$ below.

Proof : Let $v_{s}(\omega)=v(s, \omega)=\frac{\sqrt{\left|\gamma_{s}(\omega)\right|}}{\sqrt{\left|\gamma_{0}(\omega)\right|}}$. According to formula (86),

$$
\nabla_{L}\left(v^{k} U\right)=v^{k}\left(F_{1} \cdot \nabla_{L} P+F_{2} \cdot H\right)
$$

or, setting $U^{\prime}=v^{k} U, F_{1}^{\prime}=v^{k} F_{1}, F_{2}^{\prime}=v^{k} F_{2}$,

$$
\nabla_{L} U^{\prime}=F^{\prime} \nabla_{L} P+G^{\prime} \cdot H .
$$

In view of main lemma 5.5,

$$
\begin{aligned}
\left\|U^{\prime}\right\|_{\mathcal{B}^{0}} & \lesssim\left\|U^{\prime}(0)\right\|_{B_{2,1}^{0}\left(S_{0}\right)}+\left(\mathcal{N}_{1}\left(F_{1}^{\prime}\right)+\left\|F_{1}^{\prime}\right\|_{L_{x}^{\infty} L_{t}^{2}}\right) \cdot \mathcal{N}_{1}(P) \\
& +\left(\mathcal{N}_{1}\left(F_{2}^{\prime}\right)+\left\|F_{2}^{\prime}\right\|_{L_{x}^{\infty} L_{t}^{2}}\right)\|H\|_{\mathcal{P}^{0}}
\end{aligned}
$$


Now, recall that

$$
\frac{d}{d s} v=\operatorname{tr} \chi v, \quad v(0)=1
$$

We have already established a pointwise bound for $v$, see (121). We can also provide a bound for $\|\nabla v\|_{L_{x}^{2} L_{t}^{\infty}}$. Indeed, with the help of the commutation formula 2.16, $\nabla_{L} \nabla v-\nabla \frac{d}{d s} v=-\chi \cdot \nabla v$. Henceforth,

$$
\nabla_{L} \nabla v=\nabla(\operatorname{tr} \chi v)-\chi \cdot \nabla v=\frac{1}{2} \operatorname{tr} \chi \nabla v-\hat{\chi} \cdot \nabla v+v \nabla \operatorname{tr} \chi .
$$

Therefore, $\frac{d}{d s}|\nabla v|^{2}=\operatorname{tr} \chi|\nabla v|^{2}-\hat{\chi} \cdot \nabla v \cdot \nabla v$. We can now apply formula (87) to derive,

$$
|\nabla v(s, \omega)|^{2}=v(s, \omega)\left(|\nabla v(0)|^{2}+\int_{0}^{s} v\left(s^{\prime}, \omega\right)^{-1} h\left(s^{\prime}, \omega\right)\right)
$$

where $h\left(s^{\prime}, \omega\right)=-\hat{\chi} \cdot \nabla v+v \nabla \operatorname{tr} \chi$. In view of $\nabla v(0)=0$ and our bootstrap assumptions BA1 and BA2 we deduce,

$$
\begin{aligned}
\left\|\sup _{0 \leq s \leq t}|\nabla v|\right\|_{L^{2}\left(S_{0}\right)} & \lesssim\|h\|_{L_{x}^{2} L_{t}^{1}} \lesssim\|\hat{\chi}\|_{L_{x}^{\infty} L_{t}^{1}} \cdot\|\nabla v\|_{L_{t}^{\infty} L_{x}^{2}}+\|\nabla \operatorname{tr} \chi\|_{L_{x}^{2} L_{t}^{1}} \\
& \lesssim \Delta_{0}\|\nabla v\|_{L_{x}^{2} L_{t}^{\infty}}+\Delta_{0}
\end{aligned}
$$

and consequently, since $\Delta_{0}$ is sufficiently small,

$$
\|\nabla v\|_{L_{x}^{2} L_{t}^{\infty}} \leq \Delta_{0} .
$$

Using these bounds for $v$ and $\nabla v$ we infer the following,

$$
\begin{aligned}
\left\|F_{1}^{\prime}\right\|_{L_{x}^{\infty} L_{t}^{2}} & \lesssim\left\|F_{1}\right\|_{L_{x}^{\infty} L_{t}^{2}} \\
\left\|\nabla F_{1}^{\prime}\right\|_{L_{t}^{2} L_{x}^{2}} & \lesssim\left\|\nabla F_{1}\right\|_{L_{t}^{2} L_{x}^{2}}+\|\nabla v\|_{L_{x}^{2} L_{t}^{\infty}}\left\|F_{1}\right\|_{L_{x}^{\infty} L_{t}^{2}} \\
& \lesssim\left\|\nabla F_{1}\right\|_{L_{t}^{2} L_{x}^{2}}+\Delta_{0}\left\|F_{1}\right\|_{L_{x}^{\infty} L_{t}^{2}} \\
\left\|\nabla_{L} F_{1}^{\prime}\right\|_{L_{t}^{2} L_{x}^{2}} & \lesssim\left\|\nabla_{L} F_{1}\right\|_{L_{t}^{2} L_{x}^{2}}+\Delta_{0}\|F\|_{L_{x}^{\infty} L_{t}^{2}}
\end{aligned}
$$

and similar for $F_{2}^{\prime}$. Thus,

$$
\begin{aligned}
\left\|U^{\prime}\right\|_{\mathcal{B}^{0}} & \lesssim\left\|U^{\prime}(0)\right\|_{B_{2,1}^{0}\left(S_{0}\right)}+\left(\mathcal{N}_{1}\left(F_{1}\right)+\left\|F_{1}\right\|_{L_{x}^{\infty} L_{t}^{2}}\right) \cdot \mathcal{N}_{1}(P) \\
& +\left(\mathcal{N}_{1}\left(F_{2}\right)+\left\|F_{2}\right\|_{L_{x}^{\infty} L_{t}^{2}}\right)\|H\|_{\mathcal{P}^{0}}
\end{aligned}
$$

To finish it remains to check that $\|U\|_{\mathcal{B}^{0}} \lesssim\left\|U^{\prime}\right\|_{\mathcal{B}^{0}}$ and $\left\|U^{\prime}(0)\right\|_{B_{2,1}^{0}\left(S_{0}\right)} \lesssim\|U(0)\|_{B_{2,1}^{0}\left(S_{0}\right)}$. These follow easily from from the pointwise bounds on $v$ and $v^{-1}$, the $L^{2}$ bound on $\nabla v$ and a straightforward application of lemma 5.2.

The following is a also an easy consequence of part iv) of the main lemma

Proposition 5.9. Assume that the $S$ tangent tensorfield $W$ verifies the transport equation,

$$
\nabla_{L} W+k \operatorname{tr} \chi W=E
$$

for some positive $k$. Then for any other $S$ tangent tensorfield $F$,

$$
\|G \cdot W\|_{\mathcal{P}^{0}} \lesssim\left(\mathcal{N}_{1}(G)+\|G\|_{L_{x}^{\infty} L_{t}^{2}}\right) \cdot\left(\|W(0)\|_{B_{2,1}^{0}\left(S_{0}\right)}+\|E\|_{\mathcal{P}^{0}}\right)
$$


Proof : Can be proved from part iv) of the main lemma by following the same steps as in the proof of proposition 5.7.

As a corollary to proposition 5.7 we can prove the following version of the sharp classical trace theorem.

Corollary 5.10. Assume $F$ is an $S$-tangent tensor which admits a decomposition of the form, $\nabla F=\nabla_{L} P+E$, with $P, E$ tensors of the same type. Then,

$$
\|F\|_{L_{x}^{\infty} L_{t}^{2}} \lesssim \mathcal{N}_{1}(F)+\mathcal{N}_{1}(P)+\|E\|_{\mathcal{P}^{0}}
$$

Proof: The scalar function $f(t)=\int_{0}^{t}|F|^{2}$ verifies the transport equation,

$$
\nabla_{L} f=|F|^{2}, \quad U(0)=0
$$

Differentiating and applying the commutator formula (49) we derive,

$$
\begin{aligned}
\nabla_{L}(\nabla f)+\frac{1}{2} \operatorname{tr} \chi(\nabla f) & =2 F \cdot \nabla F-\hat{\chi} \cdot(\nabla f) \\
& \approx F \cdot \nabla_{L} P+F \cdot E+A \cdot(\nabla f)
\end{aligned}
$$

Applying (191) we deduce,

$$
\begin{aligned}
\|\nabla f\|_{\mathcal{B}^{0}} & \lesssim\left(\mathcal{N}_{1}(F)+\|F\|_{L_{x}^{\infty} L_{t}^{2}}\right) \cdot \mathcal{N}_{1}(P) \\
& +\left(\mathcal{N}_{1}(F)+\|F\|_{L_{x}^{\infty} L_{t}^{2}}\right) \cdot\|E\|_{\mathcal{P}^{0}} \\
& +\left(\mathcal{N}_{1}(A)+\|A\|_{L_{x}^{\infty} L_{t}^{2}}\right) \cdot\|\nabla f\|_{\mathcal{P}^{0}}
\end{aligned}
$$

Therefore in view of our bootstrap assumptions for $A$,

$$
\|\nabla f\|_{\mathcal{B}^{0}} \lesssim\left(\mathcal{N}_{1}(F)+\|F\|_{L_{x}^{\infty} L_{t}^{2}}\right) \cdot\left(\mathcal{N}_{1}(P)+\|E\|_{\mathcal{P}^{0}}\right)+\Delta_{0}\|\nabla f\|_{\mathcal{B}^{0}}
$$

which implies, for $\Delta_{0}$ sufficiently small.

$$
\|\nabla f\|_{\mathcal{B}^{0}} \lesssim\left(\mathcal{N}_{1}(F)+\|F\|_{L_{x}^{\infty} L_{t}^{2}}\right) \cdot\left(\mathcal{N}_{1}(P)+\|E\|_{\mathcal{P}^{0}}\right) .
$$

Now, in view of estimate (177), we infer that,

$$
\begin{aligned}
\|f\|_{L_{t}^{\infty} L_{x}^{\infty}} & \lesssim\|f\|_{\mathcal{B}^{1}} \lesssim\|f\|_{L_{t}^{\infty} L_{x}^{2}}+\|f\|_{\mathcal{B}^{1}} \\
& \lesssim\|f\|_{L_{t}^{\infty} L_{x}^{2}}+\left(\mathcal{N}_{1}(F)+\|F\|_{L_{x}^{\infty} L_{t}^{2}}\right) \cdot\left(\mathcal{N}_{1}(P)+\|E\|_{\mathcal{P}^{0}}\right) \\
& \lesssim\|F\|_{L_{t}^{2} L_{x}^{4}}^{2}+\left(\mathcal{N}_{1}(F)+\|F\|_{L_{x}^{\infty} L_{t}^{2}}\right) \cdot\left(\mathcal{N}_{1}(P)+\|E\|_{\mathcal{P}^{0}}\right)
\end{aligned}
$$

Thus, recalling the definition of $f=\int_{0}^{t}|F|^{2}$, and the estimate $\|F\|_{L_{t}^{2} L_{x}^{4}} \lesssim \mathcal{N}_{1}(F)$ of corollary 4.7, we infer that,

$$
\|F\|_{L_{x}^{\infty} L_{t}^{2}}^{2} \lesssim\left(\mathcal{N}_{1}(F)+\|F\|_{L_{x}^{\infty} L_{t}^{2}}\right) \cdot\left(\mathcal{N}_{1}(P)+\|E\|_{\mathcal{P}^{0}}\right)+\mathcal{N}_{1}(F)^{2}
$$

whence,

$$
\|F\|_{L_{x}^{\infty} L_{t}^{2}} \lesssim \mathcal{N}_{1}(F)+\mathcal{N}_{1}(P)+\|E\|_{\mathcal{P}^{0}}
$$

as desired. 
We shall also need elliptic estimates in Besov norms applied to the Hodge operators $\mathcal{D}_{1}, \mathcal{D}_{2},{ }^{\star} \mathcal{D}_{1},{ }^{\star} \mathcal{D}_{2}$. More precisely we want to extend the estimates of proposition 4.22 from $L^{2}(S)$ to $\mathcal{P}^{0}$. The proofs, which are quite subtle because of the commutators between the LP projections $P_{k}$ and the Hodge operators, are to be found in [Kl-Rodn3], [Kl-Rodn4].

Proposition 5.11. Let $\mathcal{D}^{-1}$ denote one of the operators $\mathcal{D}_{1}^{-1}, \mathcal{D}_{2}^{-1}$ or ${ }^{\star} \mathcal{D}_{1}^{-1},{ }^{\star} \mathcal{D}_{2}^{-1}$. Then, for a corresponding $S$-tangent tensor $F$ on $\mathcal{H}$, and any $0 \leq \theta<1$,

$$
\left\|\nabla \cdot \mathcal{D}^{-1} F\right\|_{\mathcal{P}^{\theta}} \lesssim\|F\|_{\mathcal{P}^{\theta}}
$$

We shall also need the following version of proposition 4.25.

Proposition 5.12. Let $\mathcal{D}^{-1}$ denote either $\mathcal{D}_{1}^{-1},{ }^{\star} \mathcal{D}_{1}^{-1}, \mathcal{D}_{2}^{-1}$. Then, for appropriate $S$-tangent tensors on $\mathcal{H}$ and any $1<p \leq 2$,

$$
\left\|\mathcal{D}^{-1} F\right\|_{B_{2,1}^{0}(S)} \lesssim\|F\|_{L^{p}(S)}
$$

Also, along $\mathcal{H}$,

$$
\left\|\mathcal{D}^{-1} F\right\|_{\mathcal{B}^{0}} \lesssim\|F\|_{L_{t}^{\infty} L_{x}^{p}}
$$

Moreover, for $0 \leq \theta<\frac{1}{2}$ and $\frac{2}{2-\theta}<p \leq 2$,

$$
\left\|\mathcal{D}^{-1} F\right\|_{\mathcal{P}^{\theta}} \lesssim\|F\|_{L_{t}^{2} L_{x}^{p}}
$$

The same estimates hold true if we replace $\mathcal{D}^{-1}$ by $\nabla \cdot \mathcal{D}^{-2}$ where $\mathcal{D}^{-2}$ denotes $\mathcal{D}_{2}^{-1} \cdot \mathcal{D}_{1}^{-1}$ or $\mathcal{D}_{1}^{-1} \cdot{ }^{\star} \mathcal{D}_{1}^{-1}$.

We shall also make use of the following:

Proposition 5.13. Let $F$ be an $S$-tangent tensor on $\mathcal{H}$. We have the estimate,

$$
\|F\|_{\mathcal{B}^{0}} \lesssim \mathcal{N}_{1}(F)
$$

Thus, in particular,

$$
\left\|\mathcal{D}^{-1} F\right\|_{\mathcal{B}^{0}} \lesssim\|F\|_{L_{t}^{2} L_{x}^{2}}+\left\|\nabla_{L} \cdot \mathcal{D}^{-1} F\right\|_{L_{t}^{2} L_{x}^{2}}
$$

where $\mathcal{D}^{-1}$ is one of the inverse Hodge operators $\mathcal{D}_{1}^{-1}, \mathcal{D}_{2}^{-1},{ }^{\star} \mathcal{D}_{1}^{-1},{ }^{\star} \mathcal{D}_{2}^{-1}$ encountered above. The same estimates hold true if we replace $\mathcal{D}^{-1}$ by $\nabla \cdot \mathcal{D}^{-2}$ where $\mathcal{D}^{-2}$ denotes $\mathcal{D}_{2}^{-1} \cdot \mathcal{D}_{1}^{-1}$ or $\mathcal{D}_{1}^{-1} \cdot{ }^{\star} \mathcal{D}_{1}^{-1}$.

\section{Structure of ERRor terms}

In this section we shall study various type of error terms which we will have to deal with in the next section. Some of these error terms are easy to treat while others, such as various commutators between $\nabla_{L}$ and operators of order -1 , are of the same order of regularity as the principal terms and therefore require a lot of care.

The main results of the section are proposition 6.18 and the decomposition lemma 6.13 , both will play an important role in the next section. 
We recall our main bootstrap assumptions:

$$
\begin{aligned}
\|A\|_{L_{x}^{\infty} L_{t}^{2}}, \quad\|M\|_{L_{x}^{2} L_{t}^{\infty}}, \quad \mathcal{N}_{1}(\underline{A}) & \leq \Delta_{0}, \\
\|\underline{A}\|_{L_{x}^{2} L_{t}^{\infty}}, \quad\|\underline{A}\|_{\mathcal{B}^{0}} & \leq \Delta_{0}
\end{aligned}
$$

In view of equations (143), (144) as well as propositions 4.10, 5.3 we also have,

$$
\begin{array}{rlrl}
\|A\|_{L_{t}^{\infty} L_{x}^{4}}, & \|A\|_{L_{t}^{6} L_{x}^{6}} & \lesssim \Delta_{0} & \\
\|A\|_{L_{t}^{2} L_{x}^{p}}, & \|A\|_{\mathcal{P}^{\theta}} & \lesssim \Delta_{0}, & 2 \leq p<\infty, \quad \theta<1 \\
\|A\|_{\mathcal{B}^{\theta}} & \lesssim \Delta_{0}, & 0 \leq \theta<1 / 2 .
\end{array}
$$

Also, with a constant $\mathcal{R}_{0} \leq \Delta_{0}$,

$$
\|R\|_{L_{t}^{2} L_{x}^{2}} \leq \mathcal{R}_{0}, \quad\|\check{R}\|_{L_{t}^{2} L_{x}^{2}} \leq \mathcal{R}_{0}+\Delta_{0}^{2}
$$

6.1. Symbolic notations. Throghout this section we shall denote by $\mathcal{D}^{-1}$ any of the Hodge operators $\mathcal{D}_{1}^{-1}, \mathcal{D}_{2}^{-1},{ }^{\star} \mathcal{D}_{1}^{-1},{ }^{\star} \mathcal{D}_{2}^{-1}$ encountered before. We also denote by $\mathcal{D}^{-2}$ the operators $\mathcal{D}_{2}^{-1} \cdot \mathcal{D}_{1}^{-1}$ or $\mathcal{D}_{1}^{-1} \cdot{ }^{\star} \mathcal{D}_{1}^{-1}$. To avoid confusion in the application of the Bianchi identities we need to be more precise in the meaning of expressions such as $\mathcal{D}^{-1} \check{R}$ or $\mathcal{D}^{-2} \check{R}$.

Definition 6.2. Throughout this section we use the following conventions:

- $\check{R}$ denotes either the pair $(\check{\rho},-\check{\sigma})$ or $\check{\beta}$.

- $\mathcal{D}^{-1} \check{R}$ denotes either $\mathcal{D}_{1}^{-1}(\rho,-\sigma)$ or ${ }^{\star} \mathcal{D}_{1}^{-1} \check{\beta}$.

- $\mathcal{D}^{-2} \check{R}$ denotes either $\mathcal{D}_{2}^{-1} \cdot \mathcal{D}_{1}^{-1}(\rho,-\sigma)$ or $^{\overline{31}} \mathcal{D}_{1}^{-1} \cdot{ }^{\star} \mathcal{D}_{1}^{-1} \underline{\beta}$.

- $\mathcal{D}^{-1} \cdot \nabla_{L}(\check{R})$ denotes ${ }^{\star} \mathcal{D}_{1}^{-1} \cdot \nabla_{L} \check{\beta}$ or $\mathcal{D}_{1}^{-1} L(\check{\rho},-\check{\sigma})$

- $\mathcal{D}^{-2} \cdot \nabla_{L}(\check{R})$ denotes $\mathcal{D}_{1}^{-1} \cdot{ }^{\star} \mathcal{D}_{1}^{-\overline{1}} \cdot \nabla_{L} \underline{\underline{\beta}}$, or, $\mathcal{D}_{2}^{-1} \cdot \mathcal{D}_{1}^{-1} L(\check{\rho},-\check{\sigma})$

We recall the renormalized Bianchi equations (72)- (74),

$$
\begin{aligned}
L(\check{\rho})+\frac{3}{2} \operatorname{tr} \chi \cdot \check{\rho} & =\operatorname{div} \beta-\zeta \cdot \beta+\frac{1}{2} \hat{\chi} \cdot\left(\nabla \widehat{\otimes} \zeta+\frac{1}{2}\left(\left(\operatorname{tr} \underline{\chi}+\frac{2}{r}\right)-\frac{2}{r}\right) \cdot \hat{\chi}-\zeta \widehat{\otimes} \zeta\right) . \\
L(\check{\sigma})+\frac{3}{2} \operatorname{tr} \chi \cdot \check{\sigma} & =-\operatorname{curl} \beta+\zeta \wedge \beta+\frac{1}{2} \hat{\chi} \wedge(\nabla \widehat{\otimes} \zeta-\zeta \widehat{\otimes} \zeta) \\
\nabla_{L}(\underline{\beta}) & =-\nabla \rho+(\nabla \sigma)^{\star}-2(\nabla \widehat{\otimes} \zeta) \cdot \zeta+3\left(\zeta \cdot \rho-\zeta^{\star} \sigma\right)-\operatorname{tr} \chi \underline{\beta} \\
& +2 \zeta \cdot\left(-\frac{1}{2} \operatorname{tr} \chi \cdot \underline{\hat{\chi}}-\frac{1}{2} \operatorname{tr} \underline{\chi} \cdot \hat{\chi}+\zeta \widehat{\otimes} \zeta\right)-4 \chi \cdot \underline{\hat{\chi}} \cdot \zeta
\end{aligned}
$$

which can be written schematically in the form,

$$
\begin{aligned}
\nabla_{L} \underline{\check{\beta}} & ={ }^{\star} \mathcal{D}_{1}(\rho, \sigma)+\frac{1}{r} R_{0}+A \cdot\left(R_{0}+\nabla A+A \cdot \underline{A}\right) \\
L(\check{\rho},-\check{\sigma}) & =\mathcal{D}_{1} \beta+A \cdot\left(R_{0}+\nabla A+\frac{1}{r} A+A \cdot \underline{A}\right)
\end{aligned}
$$

or, according to definition 6.2 ,

$$
\mathcal{D}^{-1} \cdot \nabla_{L} \check{R}=R_{0}+\mathcal{D}^{-1}\left(\frac{1}{r} R_{0}+A \cdot\left(R_{0}+\nabla A+\frac{1}{r} A+A \cdot \underline{A}\right)\right)
$$

\footnotetext{
${ }^{31}$ or $\mathcal{D}_{1}^{-1} \cdot J \cdot{ }^{\star} \mathcal{D}_{1}^{-1} \underline{\underline{\beta}}$ where $J$ is the involution which takes the pair $(\rho, \sigma)$ into $(-\rho, \sigma)$. This involution will appear later on but it clearly does not affect the estimates.
} 
We shall need the following commutators,

\section{Definition 6.3.}

$$
\begin{aligned}
& C_{1}(\check{R})=\nabla \cdot \mathcal{D}_{2}^{-1} \cdot\left[\nabla_{L}, \mathcal{D}_{1}^{-1}\right](\check{\rho},-\check{\sigma}) \quad \text { or } \quad \nabla \cdot\left[\nabla_{L}, \mathcal{D}_{1}^{-1}\right] \cdot{ }^{\star} \mathcal{D}_{1}^{-1}(\underline{\check{\beta}}) \\
& C_{2}(\check{R})=\nabla \cdot\left[\nabla_{L}, \mathcal{D}_{2}^{-1}\right] \cdot \mathcal{D}_{1}^{-1}(\check{\rho},-\check{\sigma}) \quad \text { or } \quad \nabla \cdot\left[\nabla_{L}, \mathcal{D}_{1}^{-1}\right] \cdot{ }^{\star} \mathcal{D}_{1}^{-1}(\check{\check{\beta}}) \\
& C_{3}(\check{R})=\left[\nabla_{L}, \nabla\right] \cdot \mathcal{D}_{2}^{-1} \cdot \mathcal{D}_{1}^{-1}(\check{\rho},-\check{\sigma}) \quad \text { or } \quad\left[\nabla_{L}, \nabla\right] \cdot \mathcal{D}_{2}^{-1} \cdot \mathcal{D}_{1}^{-1}(\underline{\check{\beta}})
\end{aligned}
$$

We shall treat the two different cases together by simply writing,

$$
\begin{aligned}
& C_{1}(\check{R})=\nabla \cdot \mathcal{D}^{-1} \cdot\left[\nabla_{L}, \mathcal{D}^{-1}\right], \quad C_{2}(\check{R})=\nabla \cdot\left[\nabla_{L}, \mathcal{D}^{-1}\right] \cdot \mathcal{D}^{-1} \\
& C_{3}(\check{R})=\left[\nabla_{L}, \nabla\right] \cdot \mathcal{D}^{-2} \check{R}
\end{aligned}
$$

We also write,

$$
C(\check{R})=\left(C_{1}(\check{R}), C_{2}(\check{R}), C_{3}(\check{R})\right) .
$$

6.4. Error type terms. To analyze various error terms which appear below we make the following definition:

Definition 6.5. We say that an expression $E$ is a strong error type term, denoted Err $_{\epsilon}$ in what follows, if

$$
\|E\|_{\mathcal{P}^{\epsilon}} \lesssim \Delta_{0}^{2}
$$

for some fixed ${ }^{32} \epsilon>0$.

We say that an expression $E$ is a weak error type term, denoted $\operatorname{Err}_{0}$ in what follows, if

$$
\|E\|_{\mathcal{P}^{0}} \lesssim \Delta_{0}^{2}
$$

Proposition 6.6. The following expressions are error type in the sense of (205)

$$
\begin{array}{rlll}
E_{1}= & \mathcal{D}^{-1}(A \cdot \nabla A), & \nabla \cdot \mathcal{D}^{-2}(A \cdot \nabla A) \\
E_{2}= & \mathcal{D}^{-1}(A \cdot R), \quad \nabla \cdot \mathcal{D}^{-2}(A \cdot R), & \mathcal{D}^{-1}\left(A \cdot \nabla \cdot \mathcal{D}^{-1} R\right) \\
E_{3}= & \mathcal{D}^{-1}(A \cdot \check{R}), \quad \nabla \cdot \mathcal{D}^{-2}(A \cdot \check{R}), & \mathcal{D}^{-1}\left(A \cdot \nabla \cdot \mathcal{D}^{-1} \check{R}\right) \\
E_{4}= & \mathcal{D}^{-1}(A \cdot A \cdot A), & \mathcal{D}^{-1}(A \cdot A \cdot \underline{A}), & \nabla \cdot \mathcal{D}^{-2}(A \cdot A \cdot A), \\
& \nabla \cdot \mathcal{D}^{-2}(A \cdot A \cdot \underline{A}) \\
E_{5}= & A \cdot A, \quad \nabla \cdot \mathcal{D}^{-1}(A \cdot A)
\end{array}
$$

The following expressions are of weak error type in the sense of (206):

$$
E_{6}=A \cdot \underline{A}, \quad \nabla \cdot \mathcal{D}^{-1}(A \cdot \underline{A}),
$$

\footnotetext{
${ }^{32}$ In fact all our strong error terms are of type $\operatorname{Err}_{\epsilon}$ with $\epsilon=\frac{1}{10}$.
} 
Proof : Recall that $\nabla \cdot \mathcal{D}_{1}^{-1}, \nabla \cdot \mathcal{D}_{2}^{-1}$ and $\nabla \cdot{ }^{\star} \mathcal{D}_{1}^{-1}$ are bounded operators on $\mathcal{P}^{\theta}$, see lemma 5.11. We shall also make use of the estimate (199) of lemma 5.12, with any $\frac{2}{2-\theta}<p \leq 2$ and $\mathcal{D}^{-1}=\mathcal{D}_{1}^{-1}, \mathcal{D}_{2}^{-1}$ or ${ }^{\star} \mathcal{D}_{1}^{-1}$,

$$
\left\|\mathcal{D}^{-1} f\right\|_{\mathcal{P}^{\theta}} \lesssim\|f\|_{L_{t}^{2} L_{x}^{p}}
$$

Also,

$$
\left\|\nabla \cdot \mathcal{D}^{-2} f\right\|_{\mathcal{P}^{\theta}} \lesssim\|f\|_{L_{t}^{2} L_{x}^{p}}
$$

Thus, for $\theta=\epsilon<\frac{1}{2}$

$$
\begin{aligned}
&\left\|E_{1}\right\|_{\mathcal{P}^{\theta}} \lesssim\|A \cdot \nabla A\|_{L_{t}^{2} L_{x}^{\frac{4}{3}}} \lesssim\|A\|_{L_{t}^{\infty} L_{x}^{4}} \cdot\|\nabla A\|_{L_{t}^{2} L_{x}^{2}} \lesssim \Delta_{0}^{2} \\
&\left\|E_{2}\right\|_{\mathcal{P}^{\theta}} \lesssim\|A \cdot R\|_{L_{t}^{2} L_{x}^{\frac{4}{3}}} \lesssim\|A\|_{L_{t}^{\infty} L_{x}^{4}} \cdot\|R\|_{L_{t}^{2} L_{x}^{2}} \lesssim \Delta_{0}^{2} \\
&\left\|E_{3}\right\|_{\mathcal{P}^{\theta}} \lesssim\|A \cdot \check{R}\|_{L_{t}^{2} L_{x}^{\frac{4}{3}}} \lesssim\|A\|_{L_{t}^{\infty} L_{x}^{4}} \cdot\|\check{R}\|_{L_{t}^{2} L_{x}^{2}} \lesssim \Delta_{0}^{2}
\end{aligned}
$$

Similarily, for $\theta=\epsilon<\frac{1}{4}$,

$$
\begin{aligned}
\left\|E_{4}\right\|_{\mathcal{P}^{\theta}} & \lesssim\|A \cdot A \cdot \underline{A}\|_{L_{t}^{2} L_{x}^{\frac{8}{7}}} \lesssim\|A\|_{L_{t}^{2} L_{x}^{8}} \cdot\|A\|_{L_{t}^{\infty} L_{x}^{4}} \cdot\|\underline{A}\|_{L_{t}^{\infty} L_{x}^{2}} \\
& \lesssim\|A\|_{L_{t}^{2} L_{x}^{8}} \cdot\|A\|_{L_{t}^{\infty} L_{x}^{4}} \cdot\|\underline{A}\|_{L_{x}^{2} L_{t}^{\infty}} \lesssim \Delta_{0}^{3}
\end{aligned}
$$

To estimate $E_{5}$ we need to apply the nonsharp product estimate (189), or (190), of proposition 5.6 as well as the boundedness of $\nabla \cdot \mathcal{D}^{-1}$ on $\mathcal{P}^{\epsilon}$,

$$
\left\|E_{5}\right\|_{\mathcal{P}^{\epsilon}} \lesssim\|A \cdot A\|_{\mathcal{P}^{\epsilon}} \lesssim \mathcal{N}_{1}(A) \cdot\|A\|_{\mathcal{B}^{\epsilon}} \lesssim \mathcal{N}_{1}(A)^{2} \lesssim \Delta_{0}^{2}
$$

as desired.

The proof that $E_{6}$ is a weak error type term is much more subtle. This will be proved only at the end of the next section by an elaborate bootstrap argument. In fact we shall assume, by the auxilliary boostrap argument BA5, that $\left\|E_{6}\right\|_{\mathcal{P}^{0}} \leq \Delta_{0}^{2}$ and show that in fact $E_{6} \leq \frac{1}{2} \Delta_{0}^{2}$. We note however that error terms of type $E_{6}$ are not needed in the discussions of this section.

Proposition 6.7. The commutator $\left[\mathcal{D}^{-1}, \nabla_{L}\right](\check{R})$ is of strong error type,

$$
\left\|\left[\mathcal{D}^{-1}, \nabla_{L}\right](\check{R})\right\|_{\mathcal{P}^{\epsilon}} \lesssim \Delta_{0}^{2}
$$

Proof : To calculate the precise form of the commutator $\left[\mathcal{D}^{-1}, \nabla_{L}\right]$ we need to make use of lemma 4.23 ,

$$
\left[\nabla_{L}, \mathcal{D}^{-1}\right]=\mathcal{D}^{-1}\left[\nabla_{L}, \mathcal{D}\right] \mathcal{D}^{-1}
$$

To calculate $\left[\nabla_{L}, \mathcal{D}\right]$ commutation formulas of proposition 2.16 which we write schematically $^{33}$, for arbitrary $S$-tangent tensor $F$ on $\mathcal{H}$ as follows,

$$
\left[\nabla_{L}, \nabla\right] F=A \cdot \nabla F+A \cdot A \cdot F+\beta \cdot F
$$

\footnotetext{
${ }^{33}$ The dot products appearing in (210) refer to apropriate tensor products and traces consistent with the precise commutation formulas of $(2.16)$.
} 
According to formulas (209), (210),

$$
\begin{aligned}
{\left[\nabla_{L}, \mathcal{D}^{-1}\right] \check{R} } & =\mathcal{D}^{-1}\left(A \cdot\left(\nabla \cdot \mathcal{D}^{-1} \check{R}\right)+A \cdot A \cdot\left(\mathcal{D}^{-1} \check{R}\right)+\beta \cdot\left(\mathcal{D}^{-1} \check{R}\right)\right) \\
& =I_{1}+I_{2}+I_{3}
\end{aligned}
$$

Therefore,

$$
\left\|\left[\nabla_{L}, \mathcal{D}^{-1}\right] \check{R}\right\|_{\mathcal{P}^{\epsilon}} \lesssim\left\|I_{1}\right\|_{\mathcal{P}^{\epsilon}}+\left\|I_{2}\right\|_{\mathcal{P}^{\epsilon}}+\left\|I_{3}\right\|_{\mathcal{P}^{\epsilon}}
$$

and

$$
\begin{aligned}
\left\|I_{1}\right\|_{\mathcal{P}^{\epsilon}} & \lesssim\left\|A \cdot\left(\nabla \cdot \mathcal{D}^{-1}\right) \check{R}\right\|_{L_{t}^{2} L_{x}^{4 / 3}} \lesssim\|A\|_{L_{t}^{\infty} L_{x}^{4}} \cdot\|\check{R}\|_{L_{t}^{2} L_{x}^{2}} \lesssim \Delta_{0}^{2} \\
\left\|I_{2}\right\|_{\mathcal{P}^{\epsilon}} & \lesssim\left\|A \cdot A \cdot \mathcal{D}^{-1} \check{R}\right\|_{L_{t}^{2} L_{x}^{4 / 3}} \lesssim\|A\|_{L_{t}^{\infty} L_{x}^{4}} \cdot\|A\|_{L_{t}^{\infty} L_{x}^{4}} \cdot\left\|\mathcal{D}^{-1} \check{R}\right\|_{L_{t}^{2} L_{x}^{4}} \\
\left\|I_{3}\right\|_{\mathcal{P}^{\epsilon}} & \lesssim\left\|\beta \cdot \Delta_{0}^{2} \cdot\right\| \mathcal{D}^{-1} \check{R} \|_{L_{t}^{2} L_{x}^{4}} \lesssim \Delta_{0}^{3} \\
& \lesssim \Delta_{0}\left\|\mathcal{D}^{-1} \check{R}\right\|_{L_{t}^{2} L_{x}^{4 / 3}} \lesssim\|R\|_{L_{t}^{2} L_{x}^{2}} \cdot\left\|\mathcal{D}^{-1} \check{R}\right\|_{L_{t}^{\infty} L_{x}^{4}}
\end{aligned}
$$

In view of $(139)\left\|\mathcal{D}^{-1} \check{R}\right\|_{L_{t}^{\infty} L_{x}^{4}} \lesssim \mathcal{N}_{1}\left(\mathcal{D}^{-1} \check{R}\right)$, whence, $\left\|I_{3}\right\|_{L_{t}^{2} L_{x}^{2}} \lesssim \Delta_{0} \cdot \mathcal{N}_{1}\left(\mathcal{D}^{-1} \check{R}\right)$. Thus, back to (212),

$$
\left\|\left[\nabla_{L}, \mathcal{D}^{-1}\right] \check{R}\right\|_{\mathcal{P}^{\epsilon}} \lesssim \Delta_{0}^{2}+\Delta_{0} \cdot \mathcal{N}_{1}\left(\mathcal{D}^{-1} \check{R}\right)
$$

To finish the proof we need the estimate $\mathcal{N}_{1}\left(\mathcal{D}^{-1} \check{R}\right) \lesssim \Delta_{0}$, to be proved in the next proposition.

Proposition 6.8. We have the following estimates:

$$
\begin{aligned}
\left\|\mathcal{D}^{-1} \nabla_{L} \check{R}\right\|_{L_{t}^{2} L_{x}^{2}} & \lesssim \mathcal{R}_{0}+\Delta_{0}^{2} \\
\left\|\left[\nabla_{L}, \mathcal{D}^{-1}\right] \check{R}\right\|_{L_{t}^{2} L_{x}^{2}} & \lesssim \Delta_{0}^{2} \\
\mathcal{N}_{1}\left(\mathcal{D}^{-1} \check{R}\right) & \lesssim \mathcal{R}_{0}+\Delta_{0}^{2}
\end{aligned}
$$

Proof : To derive (214), we use the symbolic expression of the renormalized Bianchi equations (204),

$$
\mathcal{D}^{-1} \cdot \nabla_{L} \check{R}=\check{R}+\mathcal{D}^{-1}\left(\frac{1}{r} R_{0}+A \cdot\left(R_{0}+\nabla A+\frac{1}{r} A+A \cdot \underline{A}\right)\right)
$$

Observe that, in view of the error estimates of proposition 6.6 the term

$$
\mathcal{D}^{-1}\left(A \cdot\left(R_{0}+\nabla A+\frac{1}{r} A+A \cdot \underline{A}\right)\right)
$$

is a strong error term. Therefore,

$$
\begin{aligned}
\left\|\mathcal{D}^{-1} \cdot \nabla_{L} \check{R}\right\|_{L_{t}^{2} L_{x}^{2}} & \lesssim\|R\|_{L_{t}^{2} L_{x}^{2}}+\left\|\mathcal{D}^{-1}\left(\frac{1}{r} R_{0}\right)\right\|_{L_{t}^{2} L_{x}^{2}}+\Delta_{0}^{2} \\
& \lesssim \mathcal{R}_{0}+\Delta_{0}^{2}
\end{aligned}
$$

To prove (215) we go back to (213) from where, in particular,

$$
\left\|\left[\nabla_{L}, \mathcal{D}^{-1}\right] \check{R}\right\|_{L_{t}^{2} L_{x}^{2}} \lesssim \Delta_{0}^{2}+\Delta_{0} \cdot \mathcal{N}_{1}\left(\mathcal{D}^{-1} \check{R}\right)
$$


On the other hand, according to the elliptic estimates of proposition 4.22 as well as (214)

$$
\begin{aligned}
\mathcal{N}_{1}\left(\mathcal{D}^{-1} \check{R}\right) & =\left\|\mathcal{D}^{-1} \check{R}\right\|_{L_{t}^{2} L_{x}^{2}}+\left\|\nabla \cdot \mathcal{D}^{-1} \check{R}\right\|_{L_{t}^{2} L_{x}^{2}}+\left\|\nabla_{L} \cdot \mathcal{D}^{-1} \check{R}\right\|_{L_{t}^{2} L_{x}^{2}} \\
& \lesssim\|\check{R}\|_{L_{t}^{2} L_{x}^{2}}+\left\|\mathcal{D}^{-1} \cdot \nabla_{L} \check{R}\right\|_{L_{t}^{2} L_{x}^{2}}+\left\|\left[\nabla_{L}, \mathcal{D}^{-1}\right] \check{R}\right\|_{L_{t}^{2} L_{x}^{2}} \\
& \lesssim \mathcal{R}_{0}+\Delta_{0}^{2}+\left\|\left[\nabla_{L}, \mathcal{D}^{-1}\right] \check{R}\right\|_{L_{t}^{2} L_{x}^{2}}
\end{aligned}
$$

Thus, back to $(217)$

$$
\begin{aligned}
\left\|\left[\nabla_{L}, \mathcal{D}^{-1}\right] \check{R}\right\|_{L_{t}^{2} L_{x}^{2}} & \lesssim \Delta_{0}^{2}+\Delta_{0} \cdot\left(\mathcal{R}_{0}+\Delta_{0}^{2}+\left\|\left[\nabla_{L}, \mathcal{D}^{-1}\right] \check{R}\right\|_{L_{t}^{2} L_{x}^{2}}\right) \\
& \lesssim \Delta_{0}^{2}+\Delta_{0} \cdot\left\|\left[\nabla_{L}, \mathcal{D}^{-1}\right] \check{R}\right\|_{L_{t}^{2} L_{x}^{2}}
\end{aligned}
$$

Thus, for small $\Delta_{0}$,

$$
\begin{aligned}
\left\|\left[\nabla_{L}, \mathcal{D}^{-1}\right] \check{R}\right\|_{L_{t}^{2} L_{x}^{2}} & \lesssim \Delta_{0}^{2} \\
\mathcal{N}_{1}\left(\mathcal{D}^{-1} \check{R}\right) & \lesssim \mathcal{R}_{0}+\Delta_{0}^{2}
\end{aligned}
$$

as desired.

\subsection{Preliminary estimates for the commutators $C(\check{R})$.}

In the next section we shall need an extension of proposition 6.7 to commutators of the form $C(\check{R})=\left[\nabla_{L}, \nabla \cdot \mathcal{D}^{-2}\right](\check{R})$. Unfortunately this is not possible; we need to perform in fact a nontrivial renormalization. Clearly $C(\check{R})$ can be expressed as a sum of the commutators, see definition 6.3: $C_{1}(\check{R})=\nabla \cdot \mathcal{D}^{-1} \cdot\left[\nabla_{L}, \mathcal{D}^{-1}\right]$, $C_{2}(\check{R})=\nabla \cdot\left[\nabla_{L}, \mathcal{D}^{-1}\right] \cdot \mathcal{D}^{-1}, \quad C_{3}(\check{R})=\left[\nabla_{L}, \nabla\right] \cdot \mathcal{D}^{-2} \check{R}$. Recall that

$$
C(\check{R})=\left(C_{1}(\check{R}), C_{2}(\check{R}), C_{3}(\check{R})\right)
$$

and schematically,

$$
\begin{aligned}
\nabla_{L} \cdot\left(\nabla \cdot \mathcal{D}^{-2} \check{R}\right) & =\nabla \cdot \mathcal{D}^{-2} \cdot \nabla_{L}(\check{R})+C(\check{R}) \\
\nabla \cdot \mathcal{D}^{-1} \cdot \nabla_{L} \cdot \mathcal{D}^{-1}(\check{R}) & =\nabla \cdot \mathcal{D}^{-2} \cdot \nabla_{L}(\check{R})+C(\check{R}), \\
\nabla \cdot \nabla_{L} \cdot \mathcal{D}^{-2}(\check{R}) & =\nabla \cdot \mathcal{D}^{-2} \cdot \nabla_{L}(\check{R})+C(\check{R}) .
\end{aligned}
$$

In view of lemma 4.23 and commutation formula (210),

$$
\begin{aligned}
C_{1}(\check{R})= & \nabla \cdot \mathcal{D}^{-1} \cdot\left[\nabla_{L}, \mathcal{D}^{-1}\right](\check{R})=\nabla \cdot \mathcal{D}^{-1} \cdot \mathcal{D}^{-1}\left[\nabla_{L}, \mathcal{D}\right]\left(\mathcal{D}^{-1} \check{R}\right) \\
= & \nabla \cdot \mathcal{D}^{-2}\left(A \cdot \nabla \cdot\left(\mathcal{D}^{-1} \check{R}\right)+A \cdot A \cdot\left(\mathcal{D}^{-1} \check{R}\right)+\beta \cdot\left(\mathcal{D}^{-1} \check{R}\right)\right) \\
C_{2}(\check{R}) & =\nabla \cdot\left[\nabla_{L}, \mathcal{D}^{-1}\right] \cdot \mathcal{D}^{-1}(\check{R})=\nabla \cdot \mathcal{D}^{-1} \cdot\left[\nabla_{L}, \mathcal{D}\right] \cdot \mathcal{D}^{-2}(\check{R}) \\
& =\nabla \cdot \mathcal{D}^{-1}\left(A \cdot \nabla \cdot \mathcal{D}^{-2}(\check{R})+A \cdot A \cdot \mathcal{D}^{-2}(\check{R})\right) \\
& +\nabla \cdot \mathcal{D}^{-1} \cdot\left(\beta \cdot \mathcal{D}^{-2}(\check{R})\right) \\
C_{3}(\check{R}) & =\left[\nabla_{L}, \nabla\right] \cdot \mathcal{D}^{-2}(\check{R}) \\
& =A \cdot \nabla \cdot \mathcal{D}^{-2}(\check{R})+A \cdot A \cdot \mathcal{D}^{-2}(\check{R})+\beta \cdot \mathcal{D}^{-2}(\check{R})
\end{aligned}
$$


Proposition 6.10. The commutator $C_{1}(\check{R})$ is of strong error type. The other commutators $C_{2}(\check{R}), C_{3}(\check{R})$ verify:

$$
\begin{aligned}
& C_{2}(\check{R})=\nabla \cdot \mathcal{D}^{-1} \cdot\left(\beta \cdot \mathcal{D}^{-2}(\check{R})\right)+\operatorname{Err}_{\epsilon} \\
& C_{3}(\check{R})=\beta \cdot \mathcal{D}^{-2}(\check{R})+\operatorname{Err}_{\epsilon}
\end{aligned}
$$

Proof: The three terms in the expression of $C_{1}(\check{R})$ look precisely like the terms $I_{1}, I_{2}, I_{3}$ in the formula (211), with the $\mathcal{D}^{-1}$ in front replaced by $\nabla \cdot \mathcal{D}^{-2}$, and therefore can be estimated as in (212).

To estimate the terms $J_{1}=\nabla \cdot \mathcal{D}^{-1}\left(A \cdot \nabla \cdot \mathcal{D}^{-2}(\check{R})\right)$ and $J_{2}=\nabla \cdot \mathcal{D}^{-1}\left(A \cdot A \cdot \mathcal{D}^{-2}(\check{R})\right)$ on the right hand side of (220) we make use of the nonsharp product estimate (189), or (190) of proposition 5.6 as well as the boundedness of $\nabla \cdot \mathcal{D}^{-1}$ on $\mathcal{P}^{\epsilon}$. The estimate for $J_{1}$ is similar to that of $E_{5}$ in proposition 6.6,

$$
\begin{aligned}
\left\|J_{1}\right\|_{\mathcal{P}^{\epsilon}} & \lesssim\left\|A \cdot\left(\nabla \cdot \mathcal{D}^{-2}\right) \check{R}\right\|_{\mathcal{P}^{\epsilon}} \lesssim \mathcal{N}_{1}(A) \cdot\left\|\nabla \cdot \mathcal{D}^{-2} \check{R}\right\|_{\mathcal{B}^{\epsilon}} \\
& \lesssim \Delta_{0} \cdot \mathcal{N}_{1}\left(\nabla \cdot \mathcal{D}^{-2} \check{R}\right)
\end{aligned}
$$

To estimate $J_{2}$ we also use ${ }^{34}$ the product estimate (189) as follows,

$$
\left\|J_{2}\right\|_{\mathcal{P}^{\epsilon}} \lesssim\left\|A \cdot A \cdot \mathcal{D}^{-2}(\check{R})\right\|_{\mathcal{P}^{\epsilon}} \lesssim \mathcal{N}_{2}\left(\mathcal{D}^{-2} \check{R}\right) \cdot\|A \cdot A\|_{\mathcal{P}^{\epsilon}}
$$

or, since according to proposition $6.6,\|A \cdot A\|_{\mathcal{P}^{\epsilon}} \lesssim \Delta_{0}^{2}$,

$$
\left\|J_{2}\right\|_{\mathcal{P}^{\epsilon}} \lesssim \Delta_{0}^{2} \cdot \mathcal{N}_{2}\left(\mathcal{D}^{-2} \check{R}\right)
$$

Consequently,

$$
\left\|C_{2}(\check{R})-\nabla \cdot \mathcal{D}^{-1} \cdot\left(\beta \cdot \mathcal{D}^{-2}(\check{R})\right)\right\|_{\mathcal{P}^{\epsilon}} \lesssim \Delta_{0} \cdot \mathcal{N}_{1}\left(\nabla \cdot \mathcal{D}^{-2} \check{R}\right)+\Delta_{0}^{2} \cdot \mathcal{N}_{2}\left(\mathcal{D}^{-2} \check{R}\right)
$$

The estimates for $C_{3}(\check{R})$ are exactly the same as for $C_{2}(\check{R})$. Therefore,

$$
\| C_{3}(\check{R})-\left(\beta \cdot \mathcal{D}^{-2}(\check{R}) \|_{\mathcal{P}^{\epsilon}} \lesssim \Delta_{0} \cdot \mathcal{N}_{1}\left(\nabla \cdot \mathcal{D}^{-2} \check{R}\right)+\Delta_{0}^{2} \cdot \mathcal{N}_{2}\left(\mathcal{D}^{-2} \check{R}\right)\right.
$$

Thus the proof of the proposition reduces to the following estimates:

$$
\begin{aligned}
\mathcal{N}_{1}\left(\nabla \cdot \mathcal{D}^{-2} \check{R}\right) & \lesssim \mathcal{R}_{0}+\Delta_{0}^{2} \\
\mathcal{N}_{2}\left(\mathcal{D}^{-2} \check{R}\right) & \lesssim \mathcal{R}_{0}+\Delta_{0}^{2}
\end{aligned}
$$

We shall prove in fact the following proposition.

Proposition 6.11. We have the following estimates:

$$
\begin{aligned}
\left\|\nabla \cdot \mathcal{D}^{-2} \cdot \nabla_{L} \check{R}\right\|_{L_{t}^{2} L_{x}^{2}} & \lesssim \mathcal{R}_{0}+\Delta_{0}^{2} \\
\|C(\check{R})\|_{L_{t}^{2} L_{x}^{2}} & \lesssim \Delta_{0}^{2} \\
\mathcal{N}_{1}\left(\nabla \cdot \mathcal{D}^{-2} \check{R}\right) & \lesssim \mathcal{R}_{0}+\Delta_{0}^{2} \\
\mathcal{N}_{2}\left(\mathcal{D}^{-2} \check{R}\right) & \lesssim \mathcal{R}_{0}+\Delta_{0}^{2}
\end{aligned}
$$

\footnotetext{
34 alternatively one can use (190) of the main lemma.
} 
As a corollary of the above estimates, combined with corollary 4.7, we also have,

$$
\begin{aligned}
\left\|\mathcal{D}^{-1} \check{R}\right\|_{L_{t}^{\infty} L_{x}^{4}} & \lesssim \mathcal{R}_{0}+\Delta_{0}^{2} \\
\left\|\nabla \cdot \mathcal{D}^{-2} \check{R}\right\|_{L_{t}^{\infty} L_{x}^{4}} & \lesssim \mathcal{R}_{0}+\Delta_{0}^{2} \\
\left\|\mathcal{D}^{-2} \check{R}\right\|_{L_{t}^{\infty} L_{x}^{\infty}} & \lesssim \mathcal{R}_{0}+\Delta_{0}^{2}
\end{aligned}
$$

Proof: The proof of (227) is similar to that of (214). Indeed, in view of (204),

$$
\nabla \cdot \mathcal{D}^{-2} \cdot \nabla_{L} \check{R}=\nabla \cdot \mathcal{D}^{-1} \check{R}+\nabla \cdot \mathcal{D}^{-2}\left(\frac{1}{r} R_{0}+A \cdot\left(R_{0}+\nabla A+\frac{1}{r} A+A \cdot \underline{A}\right)\right)
$$

Therefore, in view of the elliptic-Hodge estimates of proposition 4.22 and the error estimates of proposition 6.6

$$
\left\|\nabla \cdot \mathcal{D}^{-2} \cdot \nabla_{L} \check{R}\right\|_{L_{t}^{2} L_{x}^{2}} \lesssim \mathcal{R}_{0}+\Delta_{0}^{2} .
$$

as desired.

To estimate the $L^{2}$ norms of the comutators $C_{i}(\check{R}), i=1,2,3$ we make use of the estimates (225) and (226) derived in the proof of proposition 6.10,

$$
\begin{aligned}
\left\|C_{1}(\check{R})\right\|_{L_{t}^{2} L_{x}^{2}} & \lesssim \Delta_{0}^{2} \\
\left\|C_{2}(\check{R})\right\|_{L_{t}^{2} L_{x}^{2}} & \lesssim\left\|\nabla \cdot \mathcal{D}^{-1} \cdot\left(\beta \cdot \mathcal{D}^{-2}(\check{R})\right)\right\|_{L_{t}^{2} L_{x}^{2}} \\
& +\Delta_{0} \cdot \mathcal{N}_{1}\left(\nabla \cdot \mathcal{D}^{-2} \check{R}\right)+\Delta_{0}^{2} \cdot \mathcal{N}_{2}\left(\mathcal{D}^{-2} \check{R}\right) \\
\left\|C_{3}(\check{R})\right\|_{L_{t}^{2} L_{x}^{2}} & \lesssim\left\|\beta \cdot \mathcal{D}^{-2}(\check{R})\right\|_{L_{t}^{2} L_{x}^{2}} \\
& +\Delta_{0} \cdot \mathcal{N}_{1}\left(\nabla \cdot \mathcal{D}^{-2} \check{R}\right)+\Delta_{0}^{2} \cdot \mathcal{N}_{2}\left(\mathcal{D}^{-2} \check{R}\right)
\end{aligned}
$$

On the other hand, making use of the estimate $\|F\|_{L_{t}^{\infty} L_{x}^{\infty}} \lesssim \mathcal{N}_{2}(F)$ of corollary 4.7

$$
\begin{aligned}
\left\|\nabla \cdot \mathcal{D}^{-1} \cdot\left(\beta \cdot \mathcal{D}^{-2}(\check{R})\right)\right\|_{L_{t}^{2} L_{x}^{2}} & \lesssim\left\|\beta \cdot \mathcal{D}^{-2}(\check{R})\right\|_{L_{t}^{2} L_{x}^{2}} \lesssim\|\beta\|_{L_{t}^{2} L_{x}^{2}} \cdot\left\|\mathcal{D}^{-2}(\check{R})\right\|_{L_{t}^{\infty} L_{x}^{\infty}} \\
& \lesssim \Delta_{0} \cdot \mathcal{N}_{2}\left(\mathcal{D}^{-2}(\check{R})\right)
\end{aligned}
$$

Consequently,

$$
\|C(\check{R})\|_{L_{t}^{2} L_{x}^{2}} \lesssim \Delta_{0}^{2}+\Delta_{0} \cdot\left(\mathcal{N}_{1}\left(\nabla \cdot \mathcal{D}^{-2} \check{R}\right)+\mathcal{N}_{2}\left(\mathcal{D}^{-2} \check{R}\right)\right)
$$

It remains to estimate $\mathcal{N}_{1}\left(\nabla \cdot \mathcal{D}^{-2} \check{R}\right), \mathcal{N}_{2}\left(\mathcal{D}^{-2} \check{R}\right)$. From the definition of $C(\check{R})$ and the estimate $(227)$ we infer that,

$$
\begin{aligned}
\left\|\nabla \cdot \nabla_{L} \cdot \mathcal{D}^{-2} \check{R}\right\|_{L_{t}^{2} L_{x}^{2}} & \lesssim \mathcal{R}_{0}+\|C(\check{R})\|_{L_{t}^{2} L_{x}^{2}}+\Delta_{0}^{2} \\
\left\|\nabla_{L} \cdot \nabla \cdot \mathcal{D}^{-2} \check{R}\right\|_{L_{t}^{2} L_{x}^{2}} & \lesssim \mathcal{R}_{0}+\|C(\check{R})\|_{L_{t}^{2} L_{x}^{2}}+\Delta_{0}^{2}
\end{aligned}
$$

On the other hand, using also the elliptic estimates of proposition 4.24,

$$
\left\|\nabla^{2} \cdot \mathcal{D}^{-2} \check{R}\right\|_{L_{t}^{2} L_{x}^{2}} \lesssim \mathcal{R}_{0}+\Delta_{0}\|C(\check{R})\|_{L_{t}^{2} L_{x}^{2}}
$$

Therefore,

$$
\begin{aligned}
\mathcal{N}_{1}\left(\nabla \cdot \mathcal{D}^{-2} \check{R}\right) & \lesssim \mathcal{R}_{0}+\|C(\check{R})\|_{L_{t}^{2} L_{x}^{2}}+\Delta_{0}^{2} \\
\mathcal{N}_{2}\left(\mathcal{D}^{-2} \check{R}\right) & \lesssim \mathcal{R}_{0}+\|C(\check{R})\|_{L_{t}^{2} L_{x}^{2}}+\Delta_{0}^{2}
\end{aligned}
$$


Combining these with (234) and using the smallness of $\Delta_{0}$, we conclude:

$$
\begin{aligned}
\|C(\check{R})\|_{L_{t}^{2} L_{x}^{2}} & \lesssim \Delta_{0}^{2} \\
\mathcal{N}_{1}\left(\nabla \cdot \mathcal{D}^{-2} \check{R}\right) & \lesssim \mathcal{R}_{0}+\Delta_{0}^{2} \\
\mathcal{N}_{2}\left(\mathcal{D}^{-2} \check{R}\right) & \lesssim \mathcal{R}_{0}+\Delta_{0}^{2}
\end{aligned}
$$

as desired.

\subsection{Decomposition and correction estimates for $C(\check{R})$.}

The terms $\nabla \cdot \mathcal{D}^{-1} \cdot\left(\beta \cdot \mathcal{D}^{-2}(\check{R})\right)$ and $\beta \cdot \mathcal{D}^{-2}(\check{R})$ appearing on the right hand side of the formulas (222) and (223) in proposition 6.10 are not weak error type. This is a serious technical difficulty requiring another renormalization. The idea is to express both of them, modulo strong error terms, as $\nabla_{L}$ derivatives of other tensors controllable in the $\mathcal{N}_{1}$ norm. This is done in the following:

Lemma 6.13 (Decomposition Lemma). Consider the expression $\beta \cdot F$ with $F$ a tensor of arbitrary order on $\mathcal{H}$ verifying

$$
\mathcal{N}_{2}(F)<\infty \text {. }
$$

i.): We claim that,

$$
\beta=\nabla_{L}\left(\mathcal{D}_{1}^{-1}(\check{R})\right)+E_{0} \quad \text { with } \quad\left\|E_{0}\right\|_{\mathcal{P}^{\epsilon}} \lesssim \Delta_{0}^{2} .
$$

ii.) There exists a decomposition,

$$
\beta \cdot F=\nabla_{L} P+E
$$

where $P, E$ are tensors ( of the same type as $\beta \cdot F$ ) verifying,

$$
\mathcal{N}_{1}(P),\|E\|_{\mathcal{P}^{\epsilon}} \lesssim \Delta_{0} \cdot \mathcal{N}_{2}(F)
$$

iii.) There exist tensors $\bar{P}, \bar{E}$ verifying the same conditions (238) as above such that,

$$
\nabla \cdot \mathcal{D}^{-1}(\beta \cdot F)=\nabla_{L} \bar{P}+\bar{E}
$$

iv.) There exists a decomposition,

$$
\mathcal{D}^{-1}(\beta \cdot F)=\nabla_{L} \tilde{P}+\tilde{E}
$$

where $\tilde{P}, \tilde{E}$ are tensors verifying,

$$
\mathcal{N}_{2}(\tilde{P}),\|\nabla \tilde{E}\|_{\mathcal{P} \epsilon} \lesssim \Delta_{0} \cdot \mathcal{N}_{2}(F)
$$

Proof : The first statement i) follows immediately from the renormalized Bianchi, see (204), and propositions 6.6, 6.7,

$$
\beta=\mathcal{D}_{1}^{-1} \cdot \nabla_{L} \check{R}+\operatorname{Err}_{\epsilon}=\nabla_{L} \cdot \mathcal{D}_{1}^{-1}(\check{R})+\operatorname{Err}_{\epsilon}
$$

Therefore $\beta=\nabla_{L}\left(\mathcal{D}^{-1}(\check{R})\right)+E_{0}$, with $\left\|E_{0}\right\|_{\mathcal{P}^{\epsilon}} \lesssim \Delta_{0}^{2}$ as desired.

To prove ii) we use (236) and write,

$$
\beta \cdot F=\left(\nabla_{L}\left(\mathcal{D}^{-1} \check{R}\right)+E_{0}\right) \cdot F=\nabla_{L}\left(\mathcal{D}^{-1} \check{R} \cdot F\right)-\left(\mathcal{D}^{-1} \check{R}\right) \cdot \nabla_{L} F+E_{0} \cdot F
$$


We now observe that $E_{0} \cdot F$ is an error type term ; to show this we need to make use of the product estimate (189) of proposition 5.6, i.e.

$$
\left\|F \cdot E_{0}\right\|_{\mathcal{P}^{\epsilon}} \lesssim \mathcal{N}_{2}(F) \cdot\left\|E_{0}\right\|_{\mathcal{P}^{\epsilon}} \lesssim \Delta_{0} \cdot \mathcal{N}_{2}(F)
$$

We next show that $\left(\mathcal{D}^{-1} \check{R}\right) \cdot \nabla_{L} F$ is also error type. To do this we need to appeal to the bilinear estimate (190) of the proposition 5.6,

$$
\begin{aligned}
\left\|\left(\mathcal{D}^{-1} \check{R}\right) \cdot \nabla_{L} F\right\|_{\mathcal{P}^{\epsilon}} & \lesssim \mathcal{N}_{1}\left(\mathcal{D}^{-1} \check{R}\right) \cdot\left(\left\|\nabla_{L} F\right\|_{L_{t}^{2} L_{x}^{2}}+\left\|\nabla \cdot \nabla_{L} F\right\|_{L_{t}^{2} L_{x}^{2}}\right) \\
& \lesssim\left(\mathcal{R}_{0}+\Delta_{0}^{2}\right) \cdot \mathcal{N}_{2}(F) \lesssim \Delta_{0} \cdot \mathcal{N}_{2}(F)
\end{aligned}
$$

as desired. Consequently, setting $P=\left(\mathcal{D}^{-1} \check{R}\right) \cdot F$, we infer that,

$$
\beta \cdot F=\nabla_{L} P+E, \quad\|E\|_{\mathcal{P}_{\epsilon}} \lesssim \Delta_{0} \cdot \mathcal{N}_{2}(F)
$$

It remains to check that $P$ verifies (238). Using (232) and (233),

$$
\begin{aligned}
\left\|\nabla_{L} P\right\|_{L_{t}^{2} L_{x}^{2}} & \lesssim\|\beta \cdot F\|_{L_{t}^{2} L_{x}^{2}}+\|E\|_{L_{t}^{2} L_{x}^{2}} \\
& \lesssim\|\beta\|_{L_{t}^{2} L_{x}^{2}} \cdot\|F\|_{L_{t}^{\infty} L_{x}^{\infty}}+\Delta_{0} \cdot \mathcal{N}_{2}(F) \lesssim \Delta_{0} \cdot \mathcal{N}_{2}(F) \\
\|\nabla P\|_{L_{t}^{2} L_{x}^{2}} & \left.\lesssim \| \nabla \cdot \mathcal{D}^{-1} \check{R}\right) \cdot F\left\|_{L_{t}^{2} L_{x}^{2}}+\right\|\left(\mathcal{D}^{-1} \check{R}\right) \cdot \nabla F \|_{L_{t}^{2} L_{x}^{2}} \\
& \lesssim\left\|\nabla \cdot \mathcal{D}^{-1} \check{R}\right\|_{L_{t}^{2} L_{x}^{2}} \cdot\|F\|_{L_{t}^{\infty} L_{x}^{\infty}}+\|\nabla F\|_{L_{t}^{4} L_{x}^{4}}\left\|\mathcal{D}^{-1} \check{R}\right\|_{L_{t}^{4} L_{x}^{4}} \lesssim \Delta_{0} \cdot \mathcal{N}_{2}(F)
\end{aligned}
$$

Thus, $\mathcal{N}_{1}(P) \lesssim \Delta_{0} \cdot \mathcal{N}_{2}(F)$ as desired.

Part iii.) of the lemma is a little more involved as we will have to prove our decomposition (239) by an iteration procedure.

Step 1. In view of the decomposition (237) above, we have

$$
\beta \cdot H=\nabla_{L} P_{1}+E_{1}, \quad \mathcal{N}_{1}\left(P_{1}\right),\left\|E_{1}\right\|_{\mathcal{P}^{\epsilon}} \lesssim \Delta_{0}^{2}
$$

Step k. We construct by recurrence two sequences $P_{2}, P_{3} \ldots P_{k}$ and $E_{2}, E_{3} \ldots E_{k}$ such that,

$$
\begin{aligned}
\beta \cdot\left(\mathcal{D}^{-1} P_{i}\right) & =\nabla_{L} P_{i+1}+E_{i+1}, \quad i=1, \ldots k-1 \\
\mathcal{N}_{1}\left(P_{i+1}\right) & \lesssim\left(C \Delta_{0}\right)^{i} \cdot \mathcal{N}_{2}(H) \\
\left\|E_{i+1}\right\|_{\mathcal{P}^{\epsilon}} & \lesssim\left(C \Delta_{0}\right)^{i} \cdot \mathcal{N}_{2}(H)
\end{aligned}
$$

Assume $P_{i}, E_{i}$ have been already constructed for $i=2,3 \ldots k$. We construct $P_{k+1}, E_{k+1}$ by applying (237) to $\beta \cdot \mathcal{D}^{-1} P_{k}$. Thus,

$$
\beta \cdot\left(\mathcal{D}^{-1} P_{k}\right)=\nabla_{L} P_{k+1}+E_{k+1}
$$

with,

$$
\begin{aligned}
\mathcal{N}_{1}\left(P_{k+1}\right) & \lesssim \Delta_{0} \cdot \mathcal{N}_{2}\left(\mathcal{D}^{-1} P_{k}\right) \lesssim \Delta_{0} \cdot \mathcal{N}_{1}\left(P_{k}\right) \leq\left(C \Delta_{0}\right)^{k} \cdot \mathcal{N}_{2}(H) \\
\left\|E_{k+1}\right\|_{\mathcal{P}^{\epsilon}} & \lesssim \Delta_{0} \cdot \mathcal{N}_{2}\left(\mathcal{D}^{-1} P_{k}\right) \lesssim \Delta_{0} \cdot \mathcal{N}_{1}\left(P_{k}\right) \leq\left(C \Delta_{0}\right)^{k} \cdot \mathcal{N}_{2}(H)
\end{aligned}
$$

where $C$ is a universal constant. These estimates are based on the following:

Lemma 6.14. The following estimate holds for any $S$-tangent tensor $P$ on $\mathcal{H}$,

$$
\begin{aligned}
\mathcal{N}_{2}\left(\mathcal{D}^{-1} P\right) & \lesssim \mathcal{N}_{1}(P) \\
\mathcal{N}_{2}\left(\nabla \cdot \mathcal{D}^{-1} P\right) & \lesssim \mathcal{N}_{1}(P)
\end{aligned}
$$


Proof : We present the proof at the end of this section.

Now consider,

$$
\begin{aligned}
I_{k} & =\nabla \cdot \mathcal{D}^{-1}\left(\beta \cdot \mathcal{D}^{-1}\left(P_{k}\right)\right)=\nabla \cdot \mathcal{D}^{-1}\left(\nabla_{L} P_{k+1}+E_{k+1}\right) \\
& =\nabla_{L}\left(\nabla \cdot \mathcal{D}^{-1} P_{k+1}\right)+\left[\nabla \cdot \mathcal{D}^{-1}, \nabla_{L}\right] P_{k+1}+\nabla \cdot \mathcal{D}^{-1} E_{k+1}
\end{aligned}
$$

On the other hand

$$
\begin{aligned}
{\left[\nabla_{L}, \nabla \cdot \mathcal{D}^{-1}\right] P } & =\left[\nabla_{L}, \nabla\right] \cdot \mathcal{D}^{-1} P+\nabla \cdot\left[\nabla_{L}, \mathcal{D}^{-1}\right] P \\
& =A \cdot \nabla \cdot \mathcal{D}^{-1} P+A \cdot A \cdot \mathcal{D}^{-1} P+\beta \cdot \mathcal{D}^{-1} P \\
& +\nabla \cdot \mathcal{D}^{-1}\left(A \cdot \nabla \cdot \mathcal{D}^{-1} P+A \cdot A \cdot \mathcal{D}^{-1} P+\beta \cdot \mathcal{D}^{-1} P\right) \\
& =\left[\nabla_{L}, \nabla \cdot \mathcal{D}^{-1}\right]_{g} P+\beta \cdot \mathcal{D}^{-1} P+\nabla \cdot \mathcal{D}^{-1}\left(\beta \cdot \mathcal{D}^{-1} P\right)
\end{aligned}
$$

where we denote by $\left[\nabla_{L}, \nabla \cdot \mathcal{D}^{-1}\right]_{g}$ the good part of the commutator $\left[\nabla_{L}, \nabla \cdot \mathcal{D}^{-1}\right]$ i.e. the one which preserves error type terms. More precisely,

Lemma 6.15. The "good commutator" $\left[\nabla_{L}, \nabla \cdot \mathcal{D}^{-1}\right]_{g}$ verifies the following estimate,

$$
\left\|\left[\nabla_{L}, \nabla \cdot \mathcal{D}^{-1}\right]_{g} P\right\|_{\mathcal{P} \epsilon} \lesssim \Delta_{0} \cdot \mathcal{N}_{1}(P)
$$

We postpone the proof until the end of this subsection. We have shown that,

$$
\begin{aligned}
I_{k} & =\nabla_{L}\left(\nabla \cdot \mathcal{D}^{-1} P_{k+1}\right)+\beta \cdot \mathcal{D}^{-1} P_{k+1}+\nabla \cdot \mathcal{D}^{-1}\left(\beta \cdot \mathcal{D}^{-1} P_{k+1}\right) \\
& +\left[\nabla_{L}, \nabla \cdot \mathcal{D}^{-1}\right]_{g} P_{k+1}+\nabla \cdot \mathcal{D}^{-1} E_{k+1}
\end{aligned}
$$

We continue as follows,

$$
\begin{aligned}
I & =\nabla \cdot \mathcal{D}^{-1}(\beta \cdot H)=\nabla \cdot \mathcal{D}^{-1}\left(\nabla_{L} P_{1}+E_{1}\right) \\
& =\nabla_{L}\left(\nabla \cdot \mathcal{D}^{-1} P_{1}\right)+\beta \cdot \mathcal{D}^{-1} P_{1}+\nabla \cdot \mathcal{D}^{-1}\left(\beta \cdot \mathcal{D}^{-1} P_{1}\right) \\
& +\left[\nabla \cdot \mathcal{D}^{-1}, \nabla_{L}\right]_{g} P_{1}+\nabla \cdot \mathcal{D}^{-1} E_{1} \\
& =\nabla_{L}\left(\nabla \cdot \mathcal{D}^{-1} P_{1}\right)+\nabla_{L} P_{2}+E_{2}+\nabla \cdot \mathcal{D}^{-1}\left(\nabla_{L} P_{2}+E_{2}\right) \\
& +\left[\nabla \cdot \mathcal{D}^{-1}, \nabla_{L}\right]_{g} P_{1}+\nabla \cdot \mathcal{D}^{-1} E_{1} \\
& =\nabla_{L}\left(\nabla \cdot \mathcal{D}^{-1} P_{1}+P_{2}\right)+\nabla \cdot \mathcal{D}^{-1}\left(\nabla_{L} P_{2}\right)+\left[\nabla \cdot \mathcal{D}^{-1}, \nabla_{L}\right]_{g} P_{1} \\
& +E_{2}+\nabla \cdot \mathcal{D}^{-1} E_{2}+\nabla \cdot \mathcal{D}^{-1} E_{1} \\
& =\nabla_{L}\left(\bar{P}_{2}\right)+\nabla \cdot \mathcal{D}^{-1}\left(\nabla_{L} P_{2}\right)+\bar{E}_{2}
\end{aligned}
$$

i.e.,

where

$$
I=\nabla_{L}\left(\bar{P}_{2}\right)+\nabla \cdot \mathcal{D}^{-1}\left(\nabla_{L} P_{2}\right)+\bar{E}_{2}
$$

$$
\begin{aligned}
& \bar{P}_{2}=\nabla \cdot \mathcal{D}^{-1} P_{1}+P_{2} \\
& \bar{E}_{2}=\left[\nabla \cdot \mathcal{D}^{-1}, \nabla_{L}\right]_{g} P_{1}+E_{2}+\nabla \cdot \mathcal{D}^{-1} E_{2}+\nabla \cdot \mathcal{D}^{-1} E_{1}
\end{aligned}
$$

Continuing to decompose $\nabla \cdot \mathcal{D}^{-1}\left(\nabla_{L} P_{2}\right)$ as above we arrive to the following formula,

$$
\begin{aligned}
I & =\nabla_{L}\left(\bar{P}_{k}\right)+\nabla \cdot \mathcal{D}^{-1}\left(\nabla_{L} P_{k}\right)+\bar{E}_{k} \\
\bar{P}_{k} & =\nabla \cdot \mathcal{D}^{-1}\left(P_{1}+\ldots P_{k-1}\right)+P_{2}+\ldots P_{k} \\
\bar{E}_{k} & =\left[\nabla \cdot \mathcal{D}^{-1}, \nabla_{L}\right]_{g}\left(P_{1}+\ldots P_{k-1}\right)+\nabla \cdot \mathcal{D}^{-1}\left(E_{1}+\ldots E_{k}\right)+E_{2}+\ldots E_{k}
\end{aligned}
$$


SERGIU KLAINERMAN AND IGOR RODNIANSKI

Observe that, in view of lemmas (6.14), (6.15), the estimates (242), (243), and the smallness of $\Delta_{0}$,

$$
\begin{aligned}
\mathcal{N}_{1}\left(\bar{P}_{k}-\bar{P}_{j}\right) & \lesssim \mathcal{N}_{1}\left(P_{j+1}\right)+\ldots \mathcal{N}_{1}\left(P_{k}\right) \lesssim \mathcal{N}_{2}(H) \cdot \sum_{j \geq 1}(C \Delta)^{j} \lesssim \Delta_{0} \cdot \mathcal{N}_{2}(H) \\
\left\|\bar{E}_{k}-\bar{E}_{j}\right\|_{\mathcal{P}^{\epsilon}} & \lesssim\left\|E_{j+1}\right\|_{\mathcal{P}^{\epsilon}}+\ldots\left\|E_{k}\right\|_{\mathcal{P}^{\epsilon}}+\Delta_{0}\left(\mathcal{N}_{1}\left(P_{j+1}\right)+\ldots \mathcal{N}_{1}\left(P_{k}\right)\right) \\
& \lesssim \mathcal{N}_{2}(H) \cdot \sum_{j \geq 1}(C \Delta)^{j} \lesssim \Delta_{0} \cdot \mathcal{N}_{2}(H)
\end{aligned}
$$

In other words $\bar{P}_{k}$ forms a Cauchy sequence relative to the norm $\mathcal{N}_{1}$, while $\bar{E}_{k}$ forms a Cauchy sequence relative to $\mathcal{P}^{\epsilon}$. Denote by $\bar{P}$ and $\bar{E}$ the corresponding limits. Clearly

$$
\mathcal{N}_{1}(\bar{P}) \lesssim \Delta_{0} \cdot \mathcal{N}_{2}(H), \quad\|\bar{E}\|_{\mathcal{P}^{\epsilon}} \lesssim \Delta_{0} \cdot \mathcal{N}_{2}(H)
$$

We also observe that, for sufficiently small $\Delta_{0}$,

$\left\|I-\nabla_{L}\left(\bar{P}_{k}\right)-\bar{E}_{k}\right\|_{L_{t}^{2} L_{x}^{2}}=\left\|\nabla \cdot \mathcal{D}^{-1}\left(\nabla_{L} P_{k}\right)\right\|_{L_{t}^{2} L_{x}^{2}} \lesssim \mathcal{N}_{1}\left(P_{k}\right) \lesssim\left(C \Delta_{0}\right)^{k} \cdot \mathcal{N}_{2}(H) \rightarrow 0$, as $k \rightarrow \infty$. Therefore $\left\|I-\nabla_{L}(\bar{P})-\bar{E}\right\|_{L_{t}^{2} L_{x}^{2}}=0$ whence,

$$
I=\nabla_{L} \bar{P}+\bar{E}
$$

as desired.

It remains to prove the last part iv) of lemma 6.13. In view of part ii.) we can write, $\beta \cdot H=\nabla_{L} P+E$, with $\mathcal{N}_{1}(P),\|E\|_{\mathcal{P}^{\epsilon}} \lesssim \Delta_{0} \cdot \mathcal{N}_{2}(H)$. As before we can construct a sequence $P_{1}, \ldots P_{k}, \ldots$ and $E_{1}, \ldots E_{k}, \ldots$ such that

$$
\beta \cdot\left(\mathcal{D}^{-1} P_{k}\right)=\nabla_{L} P_{k+1}+E_{k+1}
$$

with,

$$
\mathcal{N}_{1}\left(P_{k+1}\right),\left\|E_{k+1}\right\|_{\mathcal{P}^{0}} \lesssim\left(C \Delta_{0}\right)^{k} \cdot \mathcal{N}_{2}(H)
$$

where $C$ is a universal constant. Consider now,

$$
\begin{aligned}
I_{k+1} & =\mathcal{D}^{-1}\left(\beta \cdot \mathcal{D}^{-1} P_{k+1}\right)=\mathcal{D}^{-1}\left(\nabla_{L} P_{k}+E_{k}\right) \\
& =\nabla_{L}\left(\mathcal{D}^{-1} P_{k}\right)+\left[\mathcal{D}^{-1}, \nabla_{L}\right] P_{k}+\mathcal{D}^{-1} E_{k} \\
& =\nabla_{L}\left(\mathcal{D}^{-1} P_{k}\right)+\mathcal{D}^{-1} E_{k}+\mathcal{D}^{-1}\left(G \cdot \nabla \cdot \mathcal{D}^{-1} P_{k}+G \cdot G \cdot \mathcal{D}^{-1} P_{k}+\beta \cdot \mathcal{D}^{-1} P_{k}\right) \\
& =\nabla_{L}\left(\mathcal{D}^{-1} P_{k}\right)+\mathcal{D}^{-1}\left(\beta \cdot \mathcal{D}^{-1} P_{k}\right)+\mathcal{D}^{-1} E_{k}+\left[\mathcal{D}^{-1}, \nabla_{L}\right]_{g} P_{k}
\end{aligned}
$$

where

$$
\left[\mathcal{D}^{-1}, \nabla_{L}\right]_{g} P_{k}=\mathcal{D}^{-1}\left(G \cdot \nabla \cdot \mathcal{D}^{-1} P_{k}+G \cdot G \cdot \mathcal{D}^{-1} P_{k}\right)
$$

verifies,

$$
\left\|\nabla \cdot\left[\mathcal{D}^{-1}, \nabla_{L}\right]_{g} P\right\|_{\mathcal{P}^{\epsilon}} \lesssim \mathcal{N}_{1}(P)
$$

as it is easy to check. The proof now follows precisely as before.

We prove lemmas $6.14,6.15$ in the next subsection. 
6.16. Proof of lemmas 6.14, 6.15. To prove the first inequality of lemma 6.14 we write,

$$
\begin{aligned}
\mathcal{N}_{2}\left(\mathcal{D}^{-1} P\right) & =\left\|\mathcal{D}^{-1} P\right\|_{L_{2}}+\left\|\nabla \mathcal{D}^{-1} P\right\|_{L_{t}^{2} L_{x}^{2}}+\left\|\nabla^{2} \mathcal{D}^{-1} P\right\|_{L_{t}^{2} L_{x}^{2}} \\
& +\left\|\nabla_{L} \mathcal{D}^{-1} P\right\|_{L_{t}^{2} L_{x}^{2}}+\left\|\nabla \nabla_{L} \mathcal{D}^{-1} P\right\|_{L_{t}^{2} L_{x}^{2}} \\
& \lesssim\|P\|_{L_{t}^{2} L_{x}^{2}}+\|\nabla P\|_{L_{t}^{2} L_{x}^{2}}+\left\|\nabla_{L} \mathcal{D}^{-1} P\right\|_{L_{t}^{2} L_{x}^{2}}+\left\|\nabla \nabla_{L} \mathcal{D}^{-1} P\right\|_{L_{t}^{2} L_{x}^{2}} \\
& \lesssim \mathcal{N}_{1}(P)+\left\|\left[\nabla_{L}, \mathcal{D}^{-1}\right] P\right\|_{L_{t}^{2} L_{x}^{2}}+\left\|\nabla \cdot\left[\nabla_{L}, \mathcal{D}^{-1}\right] P\right\|_{L_{t}^{2} L_{x}^{2}}
\end{aligned}
$$

To get the desired estimate it suffices to estimate $\left\|\nabla \cdot\left[\nabla_{L}, \mathcal{D}^{-1}\right] P\right\|_{L_{t}^{2} L_{x}^{2}}$. We calculate the commutator as before,

$$
\nabla \cdot\left[\nabla_{L}, \mathcal{D}^{-1}\right] P=\nabla \cdot \mathcal{D}^{-1}\left(A \cdot \nabla \cdot \mathcal{D}^{-1} P+A \cdot A \cdot \mathcal{D}^{-1} P+\beta \cdot \mathcal{D}^{-1} P\right)
$$

Therefore,

$$
\begin{aligned}
\left\|\nabla \cdot\left[\nabla_{L}, \mathcal{D}^{-1}\right] P\right\|_{L_{t}^{2} L_{x}^{2}} & \lesssim\left\|A \cdot \nabla \cdot \mathcal{D}^{-1} P\right\|_{L_{t}^{2} L_{x}^{2}}+\left\|A \cdot A \cdot \mathcal{D}^{-1} P\right\|_{L_{t}^{2} L_{x}^{2}}+\left\|\beta \cdot \mathcal{D}^{-1} P\right\|_{L_{t}^{2} L_{x}^{2}} \\
& \lesssim\|A\|_{L_{t}^{\infty} L_{x}^{4}} \cdot\left\|\nabla \cdot \mathcal{D}^{-1} P\right\|_{L_{t}^{2} L_{x}^{4}} \\
& +\left(\|A \cdot A\|_{L_{t}^{2} L_{x}^{2}}+\|\beta\|_{L_{t}^{2} L_{x}^{2}}\right) \cdot\left\|\mathcal{D}^{-1} P\right\|_{L_{t}^{\infty} L_{x}^{\infty}} \\
& \lesssim \Delta_{0} \cdot \mathcal{N}_{2}\left(\mathcal{D}^{-1} P\right)
\end{aligned}
$$

Hence,

$$
\mathcal{N}_{2}\left(\mathcal{D}^{-1} P\right) \lesssim \mathcal{N}_{1}(P)+\Delta_{0} \cdot \mathcal{N}_{2}\left(\mathcal{D}^{-1} P\right)
$$

and therefore $\mathcal{N}_{2}\left(\mathcal{D}^{-1} P\right) \lesssim \mathcal{N}_{1}(P)$ for sufficiently small $\Delta_{0}$. The proof of the second part of lemma 6.14 is similar but simpler.

To prove lemma 6.15 we write,

$$
\begin{aligned}
{\left[\nabla_{L}, \nabla \cdot \mathcal{D}^{-1}\right]_{g} P } & =A \cdot \nabla \cdot \mathcal{D}^{-1} P+A \cdot A \cdot \mathcal{D}^{-1} P \\
& +\nabla \cdot \mathcal{D}^{-1}\left(A \cdot \nabla \cdot \mathcal{D}^{-1} P+A \cdot A \cdot \mathcal{D}^{-1} P\right) \\
& =J_{1}+J_{2}+\nabla \cdot \mathcal{D}^{-1}\left(J_{1}+J_{2}\right)
\end{aligned}
$$

In view of the $\mathcal{P}^{\epsilon}$ boundedness of $\nabla \cdot \mathcal{D}^{-1}$ it sufffices to estimate $\left\|J_{1}\right\|_{\mathcal{P}^{\epsilon}}$ and $\left\|J_{2}\right\|_{\mathcal{P}^{\epsilon}}$. These two can be estimated exactly as in the proof of proposition 6.10. Thus, using the non sharp bilinear estimate (190) of proposition 5.6,

$$
\begin{aligned}
\left\|J_{1}\right\|_{\mathcal{P}^{\epsilon}} & =\left\|A \cdot \nabla \cdot \mathcal{D}^{-1} P\right\|_{\mathcal{P}^{\epsilon}} \lesssim N_{1}(A) \cdot\left(\left\|\nabla^{2} \cdot \mathcal{D}^{-1} P\right\|_{L_{t}^{2} L_{x}^{2}}+\left\|\nabla \cdot \mathcal{D}^{-1} P\right\|_{L_{t}^{2} L_{x}^{2}}\right) \\
& \lesssim \Delta_{0} \cdot\left(\left\|\nabla^{2} \cdot \mathcal{D}^{-1} P\right\|_{L_{t}^{2} L_{x}^{2}}+\|P\|_{L_{t}^{2} L_{x}^{2}}\right) \lesssim \Delta_{0} \cdot \mathcal{N}_{1}(P)
\end{aligned}
$$

in view of the second order elliptic estimates of proposition 4.24 .

Also, with the help of (189) of proposition 5.6 and proposition 6.6,

$$
\begin{aligned}
\left\|J_{2}\right\|_{\mathcal{P}^{\epsilon}} & =\left\|A \cdot A \cdot \mathcal{D}^{-1} P\right\|_{\mathcal{P}^{\epsilon}} \lesssim \mathcal{N}_{2}\left(\mathcal{D}^{-1} P\right) \cdot\|A \cdot A\|_{\mathcal{P}^{\epsilon}} \\
& \lesssim \Delta_{0}^{2} \cdot \mathcal{N}_{2}\left(\mathcal{D}^{-1} P\right) \lesssim \Delta_{0}^{2} \cdot \mathcal{N}_{1}(P)
\end{aligned}
$$

as desired. 
6.17. Main commutator result. Using the results of the decomposition lemma 6.13 together with proposition 6.10 we deduce that all the commutators which we have denoted by $C(\check{R})$ can be decomposed into the sum between an error type term and another which is a perfect $\nabla_{L}$ derivative. More precisely,

Proposition 6.18. All the commutators $C(\check{R})$ can be expressed as follows

$$
C(\check{R})=\nabla_{L} P+E
$$

where $P$ and $E$ are tensors verifying:

$$
\mathcal{N}_{1}(P),\|E\|_{\mathcal{P}^{\epsilon}} \lesssim \Delta_{o}^{2}
$$

for some $\epsilon>0$.

Proof : Indeed in view of proposition 6.10 all commutators we have denoted by $C(\check{R})$ can be expressed as sum of terms in $\mathcal{P}^{\epsilon}$ with the exception of terms of the type $\nabla \cdot \mathcal{D}^{-1} \cdot\left(\beta \cdot \mathcal{D}^{-2}(\check{R})\right)$ and $\beta \cdot \mathcal{D}^{-2}(\check{R})$. The proposition follows immediately by applying parts ii) and iii) of the decomposition lemma to these exceptional terms. We also need to make use of the estimate $\mathcal{N}_{2}\left(\mathcal{D}^{-2}(\check{R})\right) \lesssim \mathcal{R}_{0}+\Delta_{0}^{2}$ established in proposition 6.11 .

\section{Main Estimates}

In this section we provide the proof of theorem 3.6. To do this we write down the equations satisfied by our main quantities in a schematic form to which we can then apply the main lemma 5.5. We shall rely on all the bootstrap assumptions BA1BA3 as well as BA4. In addition we shall make the following auxilliary bootstrap assumption,

BA5.

$$
\|A \cdot \underline{A}\|_{\mathcal{P}^{0}} \leq \Delta_{0}^{2}
$$

This additional assumption will be used only to derive the desired estimates for $\zeta, \mu, \operatorname{tr} \underline{\chi}, \underline{\hat{\chi}}$ and not for $\hat{\chi}, \operatorname{tr} \chi, \nabla \operatorname{tr} \chi$. We shall show, at the end of this section, that in fact

$$
\|A \cdot \underline{A}\|_{\mathcal{P}^{0}} \lesssim \Delta_{0} \cdot\left(\mathcal{I}_{0}+\mathcal{R}_{0}+\Delta_{0}^{2}\right) \leq \frac{1}{2} \Delta_{0}^{2}
$$

and therefore BA5 was justified.

We shall make systematic use of the results of the previous section concerning error type terms.

\subsection{Estimates for $\operatorname{tr} \chi, \hat{\chi}$.}

Step 1: Estimates for $\left\|\operatorname{tr} \chi-\frac{2}{r}\right\|_{L_{t}^{\infty} L_{x}^{\infty}}$. 
Recall that $\operatorname{tr} \chi-\frac{2}{r}=(\operatorname{tr} \chi-\overline{\operatorname{tr} \chi})+\left(\overline{\operatorname{tr} \chi}-\frac{2}{r}\right)=V+W$. According to proposition $2.31 \mathrm{~V}$ and $W$ verify the transport equation,

$$
\begin{aligned}
\frac{d}{d s} W+\frac{1}{2} \operatorname{tr} \chi W & =\frac{1}{2}\left(V \cdot W+V^{2}\right)-\overline{|\hat{\chi}|^{2}} \\
\frac{d}{d s} V+\frac{1}{2}(\operatorname{tr} \chi+\overline{\operatorname{tr} \chi}) V & =-\left(|\hat{\chi}|^{2}-\overline{|\hat{\chi}|^{2}}\right)
\end{aligned}
$$

Applying lemma 3.8 as well as corollary 4.7 and remembering also the bootstarp assumption $\operatorname{tr} \chi-\frac{2}{r} \lesssim \Delta_{0}$ and $\mathcal{N}_{1}(\hat{\chi})+\|\hat{\chi}\|_{L_{x}^{\infty} L_{t}^{2}} \lesssim \Delta_{0}$, we derive,

$$
\begin{aligned}
\|V\|_{L_{x}^{\infty} L_{t}^{\infty}} & \lesssim\|V(0)\|_{L^{\infty}\left(S_{0}\right)}+\left\|\left.\hat{\chi}\right|^{2}-\overline{|\hat{\chi}|^{2}}\right\|_{L_{x}^{\infty} L_{t}^{1}}^{2} \lesssim\|V(0)\|_{L^{\infty}\left(S_{0}\right)}+\|\hat{\chi}\|_{L_{x}^{\infty} L_{t}^{2}}^{2}+\|\hat{\chi}\|_{L_{t}^{2} L_{x}^{2}} \\
& \lesssim\|V(0)\|_{L^{\infty}\left(S_{0}\right)}+\Delta_{0}^{2} \\
\|W\|_{L_{x}^{\infty} L_{t}^{\infty}} & \lesssim\|W(0)\|_{L^{\infty}\left(S_{0}\right)}+\|V \cdot W\|_{L_{x}^{\infty} L_{t}^{1}}^{2}+\|V \cdot V\|_{L_{x}^{\infty} L_{t}^{1}}^{2}+\left\|\overline{|\hat{\chi}|^{2}}\right\|_{L_{x}^{\infty} L_{t}^{1}}^{2} \\
& \lesssim\|W(0)\|_{L^{\infty}\left(S_{0}\right)}+\Delta_{0}^{2}
\end{aligned}
$$

Thus,

$$
\left\|\operatorname{tr} \chi-\frac{2}{r}\right\|_{L_{t}^{\infty} L_{x}^{\infty}} \lesssim \mathcal{I}_{0}+\Delta_{0}^{2}
$$

We can also easily check the following,

$$
\left\|\nabla_{L}\left(\operatorname{tr} \chi-\frac{2}{r}\right)\right\|_{L_{x}^{\infty} L_{t}^{1}} \lesssim \Delta_{0}^{2}
$$

\section{Step 2: Estimates for $\nabla$ tr $\chi$.}

Recall the transport equation (64) for $\nabla \operatorname{tr} \chi$,

$$
\nabla_{L}(\nabla \operatorname{tr} \chi)=-\frac{3}{2} \operatorname{tr} \chi \cdot \nabla \operatorname{tr} \chi-\hat{\chi} \cdot \nabla \operatorname{tr} \chi-2 \hat{\chi} \cdot \nabla \hat{\chi}
$$

and the Codazzi equations (67),

$$
\operatorname{div} \hat{\chi}=-\beta+\frac{1}{2} \nabla \operatorname{tr} \chi-\hat{\chi} \cdot \zeta .
$$

We need also the renormalized Bianchi equations,

$$
\begin{aligned}
& L(\check{\rho})+\frac{3}{2} \operatorname{tr} \chi \cdot \check{\rho}=\operatorname{div} \beta-\zeta \cdot \beta+\frac{1}{2} \hat{\chi} \cdot\left(\nabla \widehat{\otimes} \zeta+\frac{1}{2} \operatorname{tr} \underline{\chi} \cdot \hat{\chi}-\zeta \widehat{\otimes} \zeta\right) . \\
& L(\check{\sigma})+\frac{3}{2} \operatorname{tr} \chi \cdot \check{\sigma}=-\operatorname{curl} \beta+\zeta \wedge \beta+\frac{1}{2} \hat{\chi} \wedge(\nabla \widehat{\otimes} \zeta-\zeta \widehat{\otimes} \zeta)
\end{aligned}
$$

Schematically,

or,

$$
\mathcal{D}_{2} \beta=L(\check{\rho},-\check{\sigma})+\frac{1}{r} R_{0}+A \cdot\left(R_{0}+\frac{1}{r} A+\nabla A+A \cdot \underline{A}\right) .
$$

$$
\beta=\mathcal{D}_{2}^{-1} L(\check{\rho},-\check{\sigma})+\mathcal{D}_{2}^{-1}\left(\frac{1}{r} R_{0}+A \cdot\left(R_{0}+\frac{1}{r} A+\nabla A+A \cdot \underline{A}\right)\right)
$$

Therefore, setting $\mathcal{D}^{-2}=\mathcal{D}_{2}^{-1}{ }^{\star} \mathcal{D}_{1}^{-1}, \mathcal{D}^{-1}=\mathcal{D}_{2}^{-1}$ and $L(\check{\rho},-\check{\sigma})=\nabla_{L} \check{R}$ as in the previous section,

$$
\begin{aligned}
\hat{\chi} & =\mathcal{D}^{-2} \nabla_{L} \check{R}+\mathcal{D}^{-2}\left(\frac{1}{r} R_{0}+A \cdot\left(\frac{1}{r} A+\nabla A+A \cdot \underline{A}\right)\right) \\
& +\mathcal{D}^{-1}(\nabla \operatorname{tr} \chi+A \cdot A)
\end{aligned}
$$


or, taking the covariant derivative,

$$
\begin{aligned}
\nabla \hat{\chi} & =\nabla \cdot \mathcal{D}^{-2} \nabla_{L} \check{R}+\nabla \cdot \mathcal{D}^{-2}\left(\frac{1}{r} R_{0}+A \cdot\left(\frac{1}{r} A+\nabla A+A \cdot \underline{A}\right)\right) \\
& +\nabla \cdot \mathcal{D}^{-1}(\nabla \operatorname{tr} \chi+A \cdot A)
\end{aligned}
$$

Observe that $F_{1}=\nabla \cdot \mathcal{D}^{-2}\left(A \cdot\left(\frac{1}{r} A+\nabla A+A \cdot \underline{A}\right)\right)$ and $F_{2}=\mathcal{D}^{-1}(A \cdot A)$ are of strong error type, $\left\|F_{1}\right\|_{\mathcal{P}^{\epsilon}},\left\|F_{2}\right\|_{\mathcal{P}^{\epsilon}} \lesssim \Delta_{0}^{2}$.

Thus, since clearly $\left\|\nabla \cdot \mathcal{D}^{-2}\left(\frac{1}{r} R_{0}\right)\right\|_{\mathcal{P}^{\epsilon}} \lesssim \mathcal{R}_{0}+\Delta_{0}^{2}$,

$$
\nabla \cdot \hat{\chi}=-\nabla \cdot \mathcal{D}^{-2} \cdot \nabla_{L}(\check{R})+\nabla \cdot \mathcal{D}^{-1}(\nabla \operatorname{tr} \chi)+F, \quad\|F\|_{\mathcal{P}^{\epsilon}} \lesssim \mathcal{R}_{0}+\Delta_{0}^{2}
$$

We now write, using the commutator notation of definition 6.3 ,

$$
\nabla \cdot \mathcal{D}^{-2} \cdot \nabla_{L}(\check{R})=\nabla_{L}\left(\nabla \cdot \mathcal{D}^{-2}(\check{R})\right)+C(\check{R})
$$

According to proposition 6.10,

$$
C(\check{R})=\nabla \cdot \mathcal{D}^{-1}\left(\beta \cdot \mathcal{D}^{-2} \check{R}\right)+\beta \cdot \mathcal{D}^{-2} \check{R}+\operatorname{Err}_{\epsilon}
$$

On the other hand since, in view of the decomposition lemma 6.13, there exists tensors $P^{\prime}, E^{\prime}$ such that,

$$
\begin{aligned}
C(\check{R}) & =\nabla_{L} P^{\prime}+E^{\prime} \\
\mathcal{N}_{1}\left(P^{\prime}\right),\left\|E^{\prime}\right\|_{\mathcal{P}^{\epsilon}} & \lesssim \Delta_{0} \cdot \mathcal{N}_{2}\left(\mathcal{D}^{-2} \check{R}\right) \lesssim \Delta_{0}^{2} .
\end{aligned}
$$

with the last inequality due to proposition 6.11 . Therefore,

$$
\begin{aligned}
\nabla \cdot \mathcal{D}^{-2} \cdot \nabla_{L}(\check{R}) & =\nabla_{L} P+\operatorname{Err}_{\epsilon} \\
P & =\nabla \cdot \mathcal{D}^{-2}(\check{R})+P^{\prime} \\
\mathcal{N}_{1}(P) & \leq \mathcal{R}_{0}+\Delta_{0}^{2}
\end{aligned}
$$

Therefore, back to (251) and setting $M=\nabla \operatorname{tr} \chi$,

$$
\begin{aligned}
\nabla \cdot \hat{\chi} & =\nabla_{L} P+\nabla \cdot \mathcal{D}^{-1} M+E \\
\mathcal{N}_{1}(P),\|E\|_{\mathcal{P}^{0}} & \leq \mathcal{R}_{0}+\Delta_{0}^{2}
\end{aligned}
$$

We also rewrite the transport equation for $M=\nabla \operatorname{tr} \chi$ in the form,

$$
\nabla_{L} M+\frac{3}{2} \operatorname{tr} \chi M=A \cdot\left(\nabla_{L} P+M+\nabla \cdot \mathcal{D}^{-1} M+E\right)
$$

We are now in a position to apply proposition 5.8 to (253). Using also the boundedness of $\nabla \cdot \mathcal{D}^{-1}$ on $\mathcal{P}^{0}$ and the bootstrap bound $\mathcal{N}_{1}(A)+\|A\|_{L_{x}^{\infty} L_{t}^{2}} \lesssim \Delta_{0}$ we derive,

$$
\begin{aligned}
\|M\|_{\mathcal{B}^{0}} & \lesssim\|M(0)\|_{B_{2,1}^{0}\left(S_{0}\right)}+\Delta_{0} \cdot\left(\mathcal{N}_{1}(P)+\|M\|_{\mathcal{P}^{0}}+\left\|\nabla \cdot \mathcal{D}^{-1} M\right\|_{\mathcal{P}^{0}}+\|E\|_{\mathcal{P}^{0}}\right) \\
& \lesssim \mathcal{I}_{0}+\Delta_{0} \cdot\left(\mathcal{N}_{1}(P)+\|E\|_{\mathcal{P}^{0}}+\|M\|_{\mathcal{P}^{0}}\right) \lesssim \mathcal{I}_{0}+\Delta_{0} \cdot\left(\mathcal{R}_{0}+\Delta_{0}^{2}+\|M\|_{\mathcal{B}^{0}}\right)
\end{aligned}
$$

Thus, for small $\Delta_{0}$, we deduce

$$
\|M\|_{\mathcal{B}^{0}} \lesssim \mathcal{I}_{0}+\Delta_{0}^{2}
$$


We can also derive an estimate for $\|\nabla \operatorname{tr} \chi\|_{L_{x}^{2} L_{t}^{\infty}}$ directly from the transport equation (64) which we write in the form,

$$
\nabla_{L} M+\frac{3}{2} \operatorname{tr} \chi M=A \cdot M+A \cdot A
$$

Applying to it lemma 3.8 we derive,

$$
\begin{aligned}
\|M\|_{L_{x}^{2} L_{t}^{\infty}} & \lesssim\|M(0)\|_{L^{2}\left(S_{0}\right)}+\|A \cdot M+A \cdot A\|_{L_{x}^{2} L_{t}^{1}} \\
& \lesssim \mathcal{I}_{0}+\|M\|_{L_{x}^{2} L_{t}^{\infty}} \cdot\|A\|_{L_{x}^{\infty} L_{t}^{1}}+\|A\|_{L_{x}^{\infty} L_{t}^{2}}^{2} \\
& \lesssim \mathcal{I}_{0}+\Delta_{0} \cdot\|M\|_{L_{x}^{2} L_{t}^{\infty}}+\Delta_{0}^{2}
\end{aligned}
$$

Thus, for sufficiently small $\Delta_{0}$,

$$
\|M\|_{L_{x}^{2} L_{t}^{\infty}} \lesssim \mathcal{I}_{0}+\Delta_{0}^{2}
$$

Step 3: Estimates for $\mathcal{N}_{1}(\hat{\chi})$ and $\|\hat{\chi}\|_{L_{x}^{\infty} L_{t}^{2}}$.

We first estimate $\mathcal{N}_{1}(\hat{\chi})=\|\hat{\chi}\|_{L_{t}^{2} L_{x}^{2}}+\|\nabla \hat{\chi}\|_{L_{t}^{2} L_{x}^{2}}+\left\|\nabla_{L} \hat{\chi}\right\|_{L_{t}^{2} L_{x}^{2}}$. To estimate $\|\hat{\chi}\|_{L_{t}^{2} L_{x}^{2}}+$ $\|\nabla \hat{\chi}\|_{L_{t}^{2} L_{x}^{2}}$ we make use of the elliptic estimates of proposition 4.22 to the Codazzi equations (67),

$$
\operatorname{div} \hat{\chi}=-\beta+\frac{1}{2} \nabla \operatorname{tr} \chi-\hat{\chi} \cdot \zeta .
$$

Thus, since in view of $(254)$ we have $\|\nabla \operatorname{tr} \chi\|_{L_{t}^{2} L_{x}^{2}} \lesssim \mathcal{I}_{0}+\Delta_{0}^{2}$, we deduce

$$
\begin{aligned}
\|\hat{\chi}\|_{L_{t}^{2} L_{x}^{2}}+\|\nabla \hat{\chi}\|_{L_{t}^{2} L_{x}^{2}} & \lesssim\|\beta\|_{L_{t}^{2} L_{x}^{2}}+\|\nabla \operatorname{tr} \chi\|_{L_{t}^{2} L_{x}^{2}}+\|A \cdot A\|_{L_{t}^{2} L_{x}^{2}} \\
& \lesssim \mathcal{R}_{0}+\mathcal{I}_{0}+\Delta_{0}^{2}+\|A\|_{L_{t}^{4} L_{x}^{4}}^{2} \lesssim \mathcal{R}_{0}+\mathcal{I}_{0}+\Delta_{0}^{2}
\end{aligned}
$$

To estimate $\left\|\nabla_{L} \hat{\chi}\right\|_{L_{t}^{2} L_{x}^{2}}$ we make use of the transport equation (61)

$$
\nabla_{L} \hat{\chi}=-\operatorname{tr} \chi \cdot \hat{\chi}+\alpha
$$

Thus,

$$
\left\|\nabla_{L} \hat{\chi}\right\|_{L_{t}^{2} L_{x}^{2}} \lesssim\|\operatorname{tr} \chi\|_{L^{\infty}}\|\hat{\chi}\|_{L_{t}^{2} L_{x}^{2}}+\mathcal{R}_{0} \lesssim \mathcal{I}_{0}+\mathcal{R}_{0}
$$

Therefore,

$$
\mathcal{N}_{1}(\hat{\chi}) \lesssim \mathcal{R}_{0}+\mathcal{I}_{0}+\Delta_{0}^{2}
$$

To estimate the sharp trace norm $\|\hat{\chi}\|_{L_{x}^{\infty} L_{t}^{2}}$ we make use of corollary 5.10 and the decomposition formula (252) to deduce,

$$
\begin{aligned}
\|\hat{\chi}\|_{L_{x}^{\infty} L_{t}^{2}} & \lesssim \mathcal{N}_{1}(\hat{\chi})+\mathcal{N}_{1}(P)+\left\|\nabla \cdot \mathcal{D}^{-1} M\right\|_{\mathcal{P}^{0}}+\|E\|_{\mathcal{P}^{0}} \\
& \lesssim \mathcal{N}_{1}(\hat{\chi})+\mathcal{N}_{1}(P)+\|E\|_{\mathcal{P}^{0}}+\|M\|_{\mathcal{B}^{0}} .
\end{aligned}
$$

Therefore, in view of the estimates (254) for $M=\nabla \operatorname{tr} \chi,(252)$ for $P, E$ and (256) for $\mathcal{N}_{1}(\hat{\chi})$ already derived,

$$
\|\hat{\chi}\|_{L_{x}^{\infty} L_{t}^{2}} \lesssim \mathcal{R}_{0}+\mathcal{I}_{0}+\Delta_{0}^{2}
$$

We record the results obtained so far in the following, 
Proposition 7.2. Assuming the boot-strap assumptions BA1 -BA3 we can derive the following estimates for tr $\chi$ and $\hat{\chi}$,

$$
\begin{aligned}
\left\|\operatorname{tr} \chi-\frac{2}{r}\right\|_{L_{t}^{\infty} L_{x}^{\infty}} & \lesssim \mathcal{I}_{0}+\Delta_{0}^{2} \\
\left\|\nabla_{L}\left(\operatorname{tr} \chi-\frac{2}{r}\right)\right\|_{L_{x}^{\infty} L_{t}^{2}} & \lesssim \Delta_{0}^{2} \\
\|\nabla \operatorname{tr} \chi\|_{L_{x}^{2} L_{t}^{\infty}} & \lesssim \mathcal{I}_{0}+\Delta_{0}^{2} \\
\|\hat{\chi}\|_{L_{x}^{\infty} L_{t}^{2}}+\mathcal{N}_{1}(\hat{\chi}) & \lesssim \mathcal{I}_{0}+\mathcal{R}_{0}+\Delta_{0}^{2} \\
\|\nabla \operatorname{tr} \chi\|_{\mathcal{B}^{0}} & \lesssim \mathcal{I}_{0}+\mathcal{R}_{0}+\Delta_{0}^{2}
\end{aligned}
$$

In particular we can choose $\mathcal{I}_{0}, \mathcal{R}_{0}$ sufficiently small and check that the bootstrap assumptions BA1, BA2 for tr $\chi$ and $\hat{\chi}$ are verified with $\Delta_{0}$ replaced by $\Delta_{0} / 2$. We also deduce all the estimates for tr $\chi, \hat{\chi}$ in theorem 3.6.

\subsection{Estimates for $\mu, \nabla \zeta$.}

As in the previous section the most difficult estimate is that of $\|\mu\|_{\mathcal{B}^{0}}$. we shall start with it.

Step 1: Estimates for $\mu$.

Recall the transport equation (78) for $\mu=-\operatorname{div} \zeta+\frac{1}{2} \hat{\chi} \cdot \underline{\hat{\chi}}-\rho+|\zeta|^{2}$

$$
\begin{aligned}
\frac{d}{d s} \mu+\frac{3}{2} \operatorname{tr} \chi \mu & =\hat{\chi} \cdot(\nabla \widehat{\otimes} \zeta)+\frac{1}{2} \operatorname{tr} \chi \check{\rho}+2 \zeta \cdot \nabla \operatorname{tr} \chi \\
& -4 \hat{\chi} \cdot \zeta \cdot \zeta+\operatorname{tr} \chi|\zeta|^{2}-\frac{1}{4} \operatorname{tr} \underline{\chi}|\hat{\chi}|^{2} \\
& =\hat{\chi} \cdot(\nabla \widehat{\otimes} \zeta)+\frac{1}{2} \operatorname{tr} \chi \check{\rho}+2 \zeta \cdot \nabla \operatorname{tr} \chi+\frac{1}{r}(A \cdot A+A \cdot \underline{A}) \\
& +A \cdot A \cdot A+A \cdot A \cdot \underline{A}
\end{aligned}
$$

Remark 7.4. Clearly the terms $\frac{1}{r} A \cdot A$ and $A \cdot A \cdot A$ are better than $\frac{1}{r} A \cdot \underline{A}$, respectively $A \cdot A \cdot \underline{A}$ and therefore can be omitted. To simplify notations we shall also drop the term of the form $\frac{1}{r} A \cdot \underline{A}$. Indeed it is easier to handle than $A \cdot A \cdot \underline{A}$ and though it is only quadratic relative to the bootstrap parameter $\Delta_{0}$, unlike $A \cdot A \cdot \underline{A}$ which is cubic, this will in no way affect our proof.

Thus, schematically,

$$
\frac{d}{d s} \mu+\frac{3}{2} \operatorname{tr} \chi \mu=\hat{\chi} \cdot(\nabla \widehat{\otimes} \zeta)+\frac{1}{2} \operatorname{tr} \chi \check{\rho}+A \cdot \nabla \operatorname{tr} \chi+A \cdot A \cdot \underline{A}
$$

We now want to express the terms $\hat{\chi} \cdot(\nabla \widehat{\otimes} \zeta)$, and $\operatorname{tr} \chi \cdot \check{\rho}$ in a suitable form to which we can apply our sharp bilinear trace lemma, as we have done in the transport equation for $\nabla \operatorname{tr} \chi$ in the previous subsection. We do this with the help of the Hodge system (77):

$$
\operatorname{div} \zeta=-\mu-\check{\rho}+|\zeta|^{2}, \quad \operatorname{curl} \zeta=\check{\sigma}
$$


which we write in the form,

$$
\mathcal{D}_{1} \zeta=-(\check{\rho},-\check{\sigma})-(\mu, 0)=-(\rho,-\sigma)-(\mu, 0)+A \cdot \underline{A}
$$

or, with $M=(\mu, 0)$,

$$
\zeta=-\mathcal{D}_{1}^{-1}(\rho,-\sigma)-\mathcal{D}_{1}^{-1} M+\mathcal{D}_{1}^{-1}(A \cdot \underline{A})
$$

On the other hand we write the Bianchi equation (74) for $\underline{\check{\beta}}$,

$$
\begin{aligned}
\nabla_{L}(\underline{\tilde{\beta}}) & =-\nabla \rho+(\nabla \sigma)^{\star}-2(\nabla \widehat{\otimes} \zeta) \cdot \zeta+3\left(\zeta \cdot \rho-\zeta^{\star} \sigma\right)-\operatorname{tr} \chi \underline{\beta} \\
& +2 \zeta \cdot\left(-\frac{1}{2} \operatorname{tr} \chi \cdot \underline{\hat{\chi}}-\frac{1}{2} \operatorname{tr} \underline{\chi} \cdot \hat{\chi}+\zeta \widehat{\otimes} \zeta\right)-4 \chi \cdot \underline{\hat{\chi}} \cdot \zeta
\end{aligned}
$$

in the form, see remark 7.4,

$$
\nabla_{L} \check{\underline{\beta}}={ }^{\star} \mathcal{D}_{1}(\rho, \sigma)+\frac{1}{r} R_{0}+A \cdot\left(R_{0}+\nabla A+A \cdot \underline{A}\right)
$$

or

$$
(-\rho, \sigma)=J \cdot{ }^{\star} \mathcal{D}_{1}^{-1} \cdot \nabla_{L} \underline{\underline{\beta}}+J \cdot{ }^{\star} \mathcal{D}_{1}^{-1}\left(\frac{1}{r} R_{0}\right)+J \cdot{ }^{\star} \mathcal{D}_{1}^{-1}\left(A \cdot\left(R_{0}+\nabla A+A \cdot \underline{A}\right)\right)
$$

where $J$ is the involution $(\rho, \sigma) \longrightarrow(-\rho, \sigma)$. Therefore, combining it with (264)

$$
\begin{aligned}
\zeta & =-\mathcal{D}_{1}^{-1} \cdot J \cdot{ }^{\star} \mathcal{D}_{1}^{-1}\left(\nabla_{L} \underline{\check{\beta}}+\frac{1}{r} R_{0}\right)+\mathcal{D}_{1}^{-1} M+\mathcal{D}_{1}^{-1}(A \cdot \underline{A}) \\
& +\mathcal{D}_{1}^{-1} \cdot J \cdot{ }^{\star} \mathcal{D}_{1}^{-1}\left(A \cdot\left(R_{0}+\nabla A+A \cdot \underline{A}\right)\right) .
\end{aligned}
$$

Now introduce the operators,

$$
\mathcal{D}^{-2}=\mathcal{D}_{1}^{-1} \cdot J \cdot{ }^{\star} \mathcal{D}_{1}^{-1}, \quad \mathcal{D}^{-1}=\mathcal{D}_{1}^{-1}
$$

and observe that, see proposition 6.6 , the the term $\nabla \cdot \mathcal{D}^{-2}(A \cdot(R+\nabla A+A \cdot \underline{A}))$ is a strong error term. To deal with the term $\nabla \cdot \mathcal{D}^{-1}(A \cdot \underline{A})$ we need to make use of the auxilliary assumption BA5 made at the beginning of this section,

$$
\|A \cdot \underline{A}\|_{\mathcal{P}^{0}} \lesssim \Delta_{0}^{2}
$$

as well as the boundedness of $\nabla \cdot \mathcal{D}^{-1}$ on $\mathcal{P}^{0}$. Hence also,

$$
\left\|\nabla \cdot \mathcal{D}^{-1}(A \cdot \underline{A})\right\|_{\mathcal{P}^{0}} \lesssim \Delta_{0}^{2}
$$

Remark also that,

$$
\left\|\nabla \mathcal{D}^{-2}\left(\frac{1}{r} R_{0}\right)\right\|_{\mathcal{P}^{\epsilon}} \lesssim \mathcal{R}_{0}+\Delta_{0}^{2}
$$

Thus we can write,

$$
\nabla \zeta=-\nabla \cdot \mathcal{D}^{-2}\left(\nabla_{L} \underline{\underline{\beta}}\right)+\nabla \cdot \mathcal{D}^{-1} M+E, \quad\|E\|_{\mathcal{P}^{0}} \lesssim \mathcal{R}_{0}+\Delta_{0}^{2}
$$

Next we commute $\nabla_{L}$ with $\nabla \cdot \mathcal{D}^{-2}$ in (267) exactly as in the previous subsection. Recall that, see definition 6.3 and (218),

$$
\nabla_{L} \cdot\left(\nabla \cdot \mathcal{D}^{-2} \check{R}\right)=\nabla \cdot \mathcal{D}^{-2} \cdot \nabla_{L}(\check{R})+C(\check{R})
$$

and, see proposition 6.18 ,

$$
C(\check{R})=\nabla_{L} P^{\prime}+E^{\prime}, \quad \mathcal{N}_{1}\left(P^{\prime}\right), \quad\left\|E^{\prime}\right\|_{\mathcal{P}^{\epsilon}} \lesssim \Delta_{0}^{2} .
$$


Hence, setting $P=P^{\prime}+\nabla \cdot \mathcal{D}^{-2}(\check{R})$ and recalling the estimate, see proposition $6.11, \mathcal{N}_{1}\left(\nabla \cdot \mathcal{D}^{-2}(\check{R})\right) \lesssim \mathcal{R}_{0}+\Delta_{0}^{2}$ we can write,

$$
\begin{aligned}
\nabla \zeta & =\nabla_{L}(P)+\nabla \cdot \mathcal{D}^{-1} M+\nabla \cdot \mathcal{D}^{-2}\left(\frac{1}{r} R_{0}\right)+E, \\
\mathcal{N}_{1}(P) & \lesssim \mathcal{R}_{0}+\Delta_{0}^{2}, \quad\|E\|_{\mathcal{P}^{0}} \lesssim \mathcal{R}_{0}+\Delta_{0}^{2}
\end{aligned}
$$

Going back to the transport equation (263) for $M$ we replace $\nabla \widehat{\otimes} \zeta$ according to (268), and derive

$$
\frac{d}{d s} M+\frac{3}{2} \operatorname{tr} \chi M=A \cdot\left(\nabla_{L}(P)+\nabla \cdot \mathcal{D}^{-1} M+E\right)+\frac{1}{2} \operatorname{tr} \chi \check{\rho}+A \cdot \nabla \operatorname{tr} \chi+A \cdot A \cdot \underline{A} .
$$

Now observe that, according to the auxilliary assumption BA5, $A \cdot \underline{A}$ is a weak error type term and can be incorporated in $E$,

$$
\frac{d}{d s} M+\frac{3}{2} \operatorname{tr} \chi M=A \cdot\left(\nabla_{L}(P)+\nabla \cdot \mathcal{D}^{-1} M+\nabla \operatorname{tr} \chi+E\right)+\frac{1}{2} \operatorname{tr} \chi \check{\rho}
$$

It remains to deal with the term tr $\chi \check{\rho}$. Returning to (265) we write,

$$
\begin{aligned}
(\rho, \sigma) & ={ }^{\star} \mathcal{D}_{1}^{-1} \nabla_{L} \underline{\check{\beta}}+{ }^{\star} \mathcal{D}_{1}^{-1}\left(\frac{1}{r} R_{0}\right)+{ }^{\star} \mathcal{D}_{1}^{-1}\left(A \cdot\left(R_{0}+\nabla A+A \cdot \underline{A}\right)\right) \\
& =\nabla_{L}\left({ }^{\star} \mathcal{D}_{1}^{-1} \underline{\underline{\beta}}\right)+\left[\nabla_{L},{ }^{\star} \mathcal{D}_{1}^{-1}\right](\check{R})+{ }^{\star} \mathcal{D}_{1}^{-1}\left(\frac{1}{r} R_{0}\right) \\
& +{ }^{\star} \mathcal{D}_{1}^{-1}\left(A \cdot\left(R_{0}+\nabla A+A \cdot \underline{A}\right)\right)
\end{aligned}
$$

In view of proposition 6.7 the commutator $\left[\nabla_{L},{ }^{\star} \mathcal{D}_{1}^{-1}\right](\check{R})$ is a strong error terms and so is ${ }^{\star} \mathcal{D}_{1}^{-1}\left(A \cdot\left(R_{0}+\nabla A+A \cdot \underline{A}\right)\right.$ in view of proposition (6.6). Clearly, ${ }^{\star} \mathcal{D}_{1}^{-1}\left(\frac{1}{r} R_{0}\right) \lesssim \mathcal{R}_{0}$.

Hence,

$$
(\rho, \sigma)=\nabla_{L}\left({ }^{\star} \mathcal{D}_{1}^{-1} \underline{\check{\beta}}\right)+e^{\prime}, \quad\left\|e^{\prime}\right\|_{\mathcal{P}^{0}} \lesssim \mathcal{R}_{0}+\Delta_{0}^{2}
$$

Observe that, in view of proposition $6.8, \mathcal{N}_{1}\left({ }^{\star} \mathcal{D}_{1}^{-1} \check{\beta}\right) \lesssim \mathcal{R}_{0}+\Delta_{0}^{2}$. On the other hand $\rho$ and $\check{\rho}$ differ by a terms of the form $A \cdot \underline{A}$ which is a weak error term. We have thus established the following representation formula,

$$
\check{\rho}=\nabla_{L} p^{\prime}+e^{\prime}, \quad \mathcal{N}_{1}\left(P^{\prime}\right),\left\|e^{\prime}\right\|_{\mathcal{P}^{0}} \lesssim \mathcal{R}_{0}+\Delta_{0}^{2}
$$

Going back to (270) we deduce, schematically,

$$
\frac{d}{d s} M+\frac{3}{2} \operatorname{tr} \chi M=\left(A+\frac{1}{r}\right) \cdot \nabla_{L}(P)+A \cdot\left(\nabla \cdot \mathcal{D}^{-1} M+\nabla \operatorname{tr} \chi+E\right)+\frac{1}{r} E
$$

with $E$ verifying the estimate $\left\|e^{\prime}\right\|_{\mathcal{P}^{0}} \lesssim \mathcal{R}_{0}+\Delta_{0}^{2}$. We have just proved the following:

Proposition 7.5. The mass term $\mu=M$ verifies a transport equation of the form,

$$
\frac{d}{d s} M+\frac{3}{2} \operatorname{tr} \chi M=\left(A+\frac{1}{r}\right) \cdot \nabla_{L}(P)+A \cdot\left(\nabla \cdot \mathcal{D}^{-1} M+\nabla \operatorname{tr} \chi+E\right)+\frac{1}{r} E
$$

with $P$ and $E$ verifying,

$$
\mathcal{N}_{1}(P) \lesssim \mathcal{R}_{0}+\Delta_{0}^{2}, \quad\|E\|_{\mathcal{P}^{0}} \lesssim \mathcal{R}_{0}+\Delta_{0}^{2}
$$


We use (272) to estimate $M$ with the help of proposition 5.7. Indeed setting

$$
\begin{aligned}
& F_{1}=A+\frac{1}{r}, \quad F_{2}=\frac{1}{r}, \quad F_{3}=A \\
& W=\nabla \cdot \mathcal{D}^{-1} M+\nabla \operatorname{tr} \chi+E
\end{aligned}
$$

we can rewrite (272) in the form,

$$
\nabla_{L} M+\frac{3}{2} \operatorname{tr} \chi M=F_{1} \cdot \nabla_{L} P+F_{2} \cdot E+F_{3} \cdot W .
$$

Thus, making use of the estimates for $\|\nabla \operatorname{tr} \chi\|_{\mathcal{B}^{0}} \lesssim \mathcal{I}_{0}+\Delta_{0}^{2}$ already derived in the previous subsection,

$$
\begin{aligned}
\|M\|_{\mathcal{B}^{0}} & \lesssim\|M(0)\|_{B_{2,1}^{0}\left(S_{0}\right)}+\left(\mathcal{N}_{1}\left(F_{1}\right)+\left\|F_{1}\right\|_{L_{x}^{\infty} L_{t}^{2}}\right) \cdot \mathcal{N}_{1}(P) \\
& +\left(\mathcal{N}_{1}\left(F_{2}\right)+\left\|F_{2}\right\|_{L_{x}^{\infty} L_{t}^{2}}\right)\|E\|_{\mathcal{P}^{0}}+\left(\mathcal{N}_{1}\left(F_{3}\right)+\left\|F_{3}\right\|_{L_{x}^{\infty} L_{t}^{2}}\right)\|H\|_{\mathcal{P}^{0}} \\
& \lesssim\|M(0)\|_{B_{2,1}^{0}\left(S_{0}\right)}+\mathcal{R}_{0}+\Delta_{0}^{2}+\Delta_{0} \cdot\|W\|_{\mathcal{P}^{0}} \\
\|W\|_{\mathcal{P}^{0}} & \lesssim\|M\|_{\mathcal{P}^{0}}+\|\nabla \operatorname{tr} \chi\|_{\mathcal{P}^{0}}+\mathcal{R}_{0}+\Delta_{0}^{2} \lesssim\|M\|_{\mathcal{B}^{0}}+\mathcal{I}_{0}+\mathcal{R}_{0}+\Delta_{0}^{2}
\end{aligned}
$$

Hence, $\|M\|_{\mathcal{B}^{0}} \lesssim \mathcal{I}_{0}+\Delta_{0} \cdot\|M\|_{\mathcal{B}^{0}}+\mathcal{R}_{0}+\Delta_{0}^{2}$ and therefore,

$$
\|M\|_{\mathcal{B}^{0}} \lesssim \mathcal{I}_{0}+\mathcal{R}_{0}+\Delta_{0}^{2}
$$

As in the previous subsection we need to derive also an estimate for $\|M\|_{L_{x}^{2} L_{t}^{\infty}}$. We do this with the help of lemma 3.8 applied to the transport equation (263).

$$
\begin{aligned}
\|M\|_{L_{x}^{2} L_{t}^{\infty}} & \lesssim\|M(0)\|_{L^{2}\left(S_{0}\right)}+\mathcal{R}_{0}+\|\hat{\chi} \cdot \nabla \zeta\|_{L_{x}^{2} L_{t}^{1}} \\
& +\|G \cdot \nabla \operatorname{tr} \chi\|_{L_{x}^{2} L_{t}^{1}}+\|G \cdot G \cdot B\|_{L_{x}^{2} L_{t}^{1}} \\
& \lesssim \mathcal{I}_{0}+\mathcal{R}_{0}+\Delta_{0}^{2}
\end{aligned}
$$

Hence,

$$
\|M\|_{L_{x}^{2} L_{t}^{\infty}} \lesssim \mathcal{I}_{0}+\mathcal{R}_{0}+\Delta_{0}^{2}
$$

Step 2: Estimates for $\mathcal{N}_{1}(\zeta)$. To estimate $\|\zeta\|_{L_{t}^{2} L_{x}^{2}}+\|\nabla \zeta\|_{L_{t}^{2} L_{x}^{2}}$ we simply apply the elliptic estimates of proposition 4.22 to the elliptic system (264). Thus,

$$
\begin{aligned}
\|\zeta\|_{L_{t}^{2} L_{x}^{2}}+\|\nabla \zeta\|_{L_{t}^{2} L_{x}^{2}} & \lesssim\|R\|_{L_{t}^{2} L_{x}^{2}}+\|M\|_{L_{t}^{2} L_{x}^{2}}+\|A \cdot \underline{A}\|_{L_{t}^{2} L_{x}^{2}} \\
& \lesssim \mathcal{I}_{0}+\mathcal{R}_{0}+\Delta_{0}^{2}+\|A\|_{L_{x}^{\infty} L_{t}^{2}} \cdot\|\underline{A}\|_{L_{x}^{2} L_{t}^{\infty}} \\
& \lesssim \mathcal{I}_{0}+\mathcal{R}_{0}+\Delta_{0}^{2}
\end{aligned}
$$

Also, from (63)

$$
\nabla_{L} \zeta+\operatorname{tr} \chi \zeta=R+A \cdot \underline{A}
$$

and,

$$
\left\|\nabla_{L} \zeta\right\|_{L_{t}^{2} L_{x}^{2}} \lesssim\|R\|_{L_{t}^{2} L_{x}^{2}}+\|\zeta\|_{L_{t}^{2} L_{x}^{2}}+\mathcal{R}_{0}+\Delta_{0}^{2} \lesssim \mathcal{I}_{0}+\mathcal{R}_{0}+\Delta_{0}^{2}
$$

Thus,

$$
\mathcal{N}_{1}(\zeta) \lesssim \mathcal{I}_{0}+\mathcal{R}_{0}+\Delta_{0}^{2}
$$

Step 3: Estimates for $\|\zeta\|_{L_{x}^{\infty} L_{t}^{2}}$ 
We take advantage once more of the decomposition (268),

$$
\begin{aligned}
\nabla \zeta & =\nabla_{L}(P)+\nabla \cdot \mathcal{D}^{-1} M+\nabla \cdot \mathcal{D}^{-2}\left(\frac{1}{r} R_{0}\right)+E, \\
\mathcal{N}_{1}(P) & \lesssim \mathcal{R}_{0}+\Delta_{0}^{2}, \quad\|E\|_{\mathcal{P}^{0}} \lesssim \mathcal{R}_{0}+\Delta_{0}^{2}
\end{aligned}
$$

and corollary 5.10:

$$
\begin{aligned}
\|\zeta\|_{L_{x}^{\infty} L_{t}^{2}} & \lesssim \mathcal{N}_{1}(\zeta)+\mathcal{N}_{1}(P)+\left\|\nabla \cdot \mathcal{D}^{-1} M+\nabla \cdot \mathcal{D}^{-2}\left(\frac{1}{r} R_{0}\right)+E\right\|_{\mathcal{P}^{0}} \\
& \lesssim \mathcal{I}_{0}+\mathcal{R}_{0}+\Delta_{0}^{2}
\end{aligned}
$$

We gather all the results obtained above in the following:

Proposition 7.6. Assuming the boot-strap assumptions BA1 -BA4 as well as the auxilliary bootstrap assumption BA5 we can derive the following estimates for $\mu$, $\zeta$ :

$$
\begin{aligned}
\|\mu\|_{L_{x}^{2} L_{t}^{\infty}} & \lesssim \mathcal{I}_{0}+\Delta_{0}^{2} \\
\mathcal{N}_{1}(\zeta) & \lesssim \mathcal{I}_{0}+\mathcal{R}_{0}+\Delta_{0}^{2} \\
\|\zeta\|_{L_{x}^{\infty} L_{t}^{2}} & \lesssim \mathcal{I}_{0}+\mathcal{R}_{0}+\Delta_{0}^{2} \\
\|\mu\|_{\mathcal{B}^{0}} & \lesssim \mathcal{I}_{0}+\mathcal{R}_{0}+\Delta_{0}^{2}
\end{aligned}
$$

In particular we can choose $\mathcal{I}_{0}, \mathcal{R}_{0}$ sufficiently small and check that the bootstrap assumptions BA1, BA2 for $\zeta$ and $\mu$ are verified with $\Delta_{0}$ replaced by $\Delta_{0} / 2$. We also deduce all the estimates for $\zeta, \mu$ in theorem 3.6.

\subsection{Estimates for $(\operatorname{tr} \underline{\chi}, \underline{\hat{\chi}})$.}

Step 1: $\quad \mathcal{B}^{0}$ estimates for $(\operatorname{tr} \underline{\underline{\chi}}, \underline{\hat{\chi}})$

Recall the transport equations (65), (66) and Codazzi equations (69).

$$
\begin{aligned}
\frac{d}{d s} \operatorname{tr} \underline{\chi} & =-2 \operatorname{div} \zeta-\chi \cdot \underline{\chi}+2|\zeta|^{2}+2 \rho \\
\nabla_{L} \underline{\hat{\chi}} & =-\nabla \hat{\otimes} \zeta-\frac{1}{2}(\operatorname{tr} \underline{\chi} \cdot \underline{\hat{\chi}}+\operatorname{tr} \underline{\chi} \cdot \hat{\chi})+\zeta \widehat{\otimes} \zeta \\
\operatorname{div} \underline{\hat{\chi}} & =\frac{1}{2} \nabla \operatorname{tr} \underline{\chi}-\frac{1}{2} \operatorname{tr} \underline{\chi} \cdot \zeta+\zeta \cdot \underline{\hat{\chi}}+\underline{\beta} \\
& =\frac{1}{2} \nabla \operatorname{tr} \underline{\chi}-\frac{1}{2} \operatorname{tr} \underline{\chi} \cdot \zeta-\zeta \cdot \underline{\hat{\chi}}+\underline{\underline{\beta}}
\end{aligned}
$$

Recall that $\mu=-\operatorname{div} \zeta+\frac{1}{2} \hat{\chi} \cdot \underline{\hat{\chi}}-\rho+|\zeta|^{2}$. Thus,

$$
\frac{d}{d s} \operatorname{tr} \underline{\chi}+\frac{1}{2} \operatorname{tr} \chi \cdot \operatorname{tr} \underline{\chi}=2 \mu-2 \hat{\chi} \cdot \underline{\hat{\chi}}+4 \rho
$$

Also, since $\frac{d}{d s} \operatorname{tr} \chi+\frac{1}{2} \operatorname{tr} \chi^{2}=-|\hat{\chi}|^{2}$,

$$
\frac{d}{d s}(\operatorname{tr} \underline{\chi}+\operatorname{tr} \chi)+\frac{1}{2} \operatorname{tr} \chi(\operatorname{tr} \underline{\chi}+\operatorname{tr} \chi)=2 \mu+4 \rho-2 \hat{\chi} \cdot \underline{\hat{\chi}}-\hat{\chi} \cdot \hat{\chi}
$$


On the other hand, recalling (271)

$$
\begin{gathered}
\rho=\nabla_{L} p+e^{\prime}, \quad \mathcal{N}_{1}(p),\left\|e^{\prime}\right\|_{\mathcal{P}^{0}} \lesssim \mathcal{R}_{0}+\Delta_{0}^{2} \\
\frac{d}{d s}(\operatorname{tr} \chi+\operatorname{tr} \underline{\chi})+\frac{1}{2} \operatorname{tr} \chi(\operatorname{tr} \underline{\chi}+\operatorname{tr} \chi)=2 \mu+4 \nabla_{L} p+\hat{\chi} \cdot \underline{\hat{\chi}}+e^{\prime}
\end{gathered}
$$

Observe that according to the auxilliary assumption BA5 we have $\|\chi \cdot \underline{\chi}\|_{\mathcal{P}^{0}} \lesssim \Delta_{0}^{2}$ while, in view of the estimates of the previous section $\|\mu\|_{\mathcal{P}^{0}} \lesssim \mathcal{I}_{0}+\mathcal{R}_{0}+\Delta_{0}^{2}$. Therefore introducing $e=2 \mu+e^{\prime}+\chi \cdot \underline{\chi}$ and $b=\operatorname{tr} \chi+\operatorname{tr} \underline{\chi}$, we derive:

$$
\frac{d}{d s} b+\frac{1}{2} \operatorname{tr} \chi b=4 \nabla_{L} p+e
$$

where

$$
\mathcal{N}_{1}(p), \quad\|e\|_{\mathcal{P}^{0}} \lesssim \mathcal{I}_{0}+\mathcal{R}_{0}+\Delta_{0}^{2}
$$

We can write (281) in the form,

$$
\frac{d}{d s} b+\frac{1}{2} \operatorname{tr} \chi b=F_{1} \cdot \nabla_{L} p+F_{2} \cdot e
$$

where $F_{1}=4,, F_{2}=1, b=\operatorname{tr} \chi+\operatorname{tr} \underline{\chi}$, and apply proposition 5.7.

$$
\begin{aligned}
\|b\|_{\mathcal{B}^{0}} & \lesssim\|b(0)\|_{B_{2,1}^{0}\left(S_{0}\right)}+\mathcal{N}_{1}(p)+\|e\|_{\mathcal{P}^{0}} \\
& \lesssim \mathcal{I}_{0}+\mathcal{R}_{0}+\Delta_{0}^{2} \lesssim \mathcal{I}_{0}+\mathcal{R}_{0}+\Delta_{0}^{2}
\end{aligned}
$$

We can proceed exactly in the same manner with the transport equation for $\underline{\hat{\chi}}$,

$$
\begin{aligned}
\nabla_{L} \underline{\hat{\chi}}+\frac{1}{2} \operatorname{tr} \chi \cdot \underline{\hat{\chi}} & =-\nabla \widehat{\otimes} \zeta-\frac{1}{2} \operatorname{tr} \underline{\chi} \cdot \hat{\chi}+\zeta \widehat{\otimes} \zeta \\
& =-\nabla \widehat{\otimes} \zeta-\frac{1}{2} b \cdot \hat{\chi}+\frac{1}{r} \cdot \hat{\chi}+A \cdot A
\end{aligned}
$$

or, recalling (268),

$$
\begin{aligned}
\nabla \widehat{\otimes} \zeta & =\nabla_{L}(P)+\nabla \cdot \mathcal{D}^{-1} \mu+\nabla \cdot \mathcal{D}^{-2}\left(\frac{1}{r} R_{0}\right)+E^{\prime}, \\
\mathcal{N}_{1}(P) & \lesssim \mathcal{R}_{0}+\Delta_{0}^{2}, \quad\left\|E^{\prime}\right\|_{\mathcal{P}^{0}} \lesssim \mathcal{R}_{0}+\Delta_{0}^{2}
\end{aligned}
$$

Therefore, writing $\operatorname{tr} \underline{\chi}=b-\operatorname{tr} \chi=b-\left(\operatorname{tr} \chi-\frac{2}{r}\right)+\frac{2}{r}$ and including $b$ among the terms we have denoted by $\underline{A}$, we can write,

$$
\nabla_{L}(\underline{\hat{\chi}})+\frac{1}{2} \operatorname{tr} \chi \cdot \underline{\hat{\chi}}=-\nabla_{L} P+\nabla \cdot \mathcal{D}^{-1} \mu+\frac{1}{r}\left(\hat{\chi}+\nabla \cdot \mathcal{D}^{-2}\left(R_{0}\right)\right)+A \cdot \underline{A}+E^{\prime}
$$

Observe that the term

$$
E=\nabla \cdot \mathcal{D}^{-1} \mu+\frac{1}{r}\left(\hat{\chi}+\nabla \cdot \mathcal{D}^{-2}\left(R_{0}\right)\right)+A \cdot \underline{A}+E^{\prime}
$$

verifies the estimate,

$$
\begin{aligned}
\|E\|_{\mathcal{P}^{0}} & \lesssim\|\mu\|_{\mathcal{P}^{0}}+\|\hat{\chi}\|_{\mathcal{P}^{0}}+\left\|\nabla \cdot \mathcal{D}^{-2}\left(R_{0}\right)\right\|_{\mathcal{P}^{0}}+\|A \cdot \underline{A}\|_{\mathcal{P}^{0}}+\left\|E^{\prime}\right\|_{\mathcal{P}^{0}} \\
& \lesssim \mathcal{I}_{0}+\mathcal{R}_{0}+\Delta_{0}^{2}
\end{aligned}
$$

Therefore,

$$
\begin{aligned}
\nabla_{L}(\underline{\hat{\chi}})+\frac{1}{2} \operatorname{tr} \chi \cdot \hat{\chi} & =-\nabla_{L} P+E \\
\mathcal{N}_{1}(P),\|E\|_{\mathcal{P}^{0}} & \lesssim \mathcal{I}_{0}+\mathcal{R}_{0}+\Delta_{0}^{2}
\end{aligned}
$$


to which we can apply as before proposition 5.7 to derive,

$$
\begin{aligned}
\|\underline{\hat{\chi}}\|_{\mathcal{B}^{0}} & \lesssim\|\underline{\hat{\chi}}(0)\|_{B_{2,1}^{0}\left(S_{0}\right)}+\mathcal{N}_{1}(P)+\|E\|_{\mathcal{P}^{0}} \\
& \lesssim \mathcal{I}_{0}+\mathcal{R}_{0}+\Delta_{0}^{2}
\end{aligned}
$$

i.e.,

$$
\|\underline{\hat{\chi}}\|_{\mathcal{B}^{0}} \lesssim \mathcal{I}_{0}+\mathcal{R}_{0}+\Delta_{0}^{2}
$$

Step 2: Estimates in the norm $L_{x}^{2} L_{t}^{\infty}$ We shall prove the following estimate:

$$
\left\|\operatorname{tr} \underline{\chi}+\frac{2}{r}\right\|_{L_{x}^{2} L_{t}^{\infty}}+\|\underline{\hat{\chi}}\|_{L_{x}^{2} L_{t}^{\infty}} \lesssim \mathcal{I}_{0}+\Delta_{0}^{2}
$$

We consider once more the transport equation (280)

$$
\frac{d}{d s}(\operatorname{tr} \underline{\chi}+\operatorname{tr} \chi)+\frac{1}{2} \operatorname{tr} \chi(\operatorname{tr} \underline{\underline{\chi}}+\operatorname{tr} \chi)=2 \mu-4 \rho+A \cdot \underline{A},
$$

and apply to it lemma 3.8. Taking also into account the estimates for $\mu$ derived in the previous subsection,

$$
\begin{aligned}
\|\operatorname{tr} \chi+\operatorname{tr} \underline{\chi}\|_{L_{x}^{2} L_{t}^{\infty}} & \lesssim\|(\operatorname{tr} \chi+\operatorname{tr} \underline{\chi})(0)\|_{L_{x}^{2}\left(S_{0}\right)}+\|\mu\|_{L_{x}^{2} L_{t}^{1}}+\mathcal{R}_{0} \\
& +\|A \cdot \underline{A}\|_{L_{x}^{2} L_{t}^{1}} \lesssim \mathcal{I}_{0}+\mathcal{R}_{0}+\Delta_{0}^{2}
\end{aligned}
$$

Thus,

$$
\begin{aligned}
\left\|\operatorname{tr} \underline{\chi}+\frac{2}{r}\right\|_{L_{x}^{2} L_{t}^{\infty}} & \lesssim\left\|\operatorname{tr} \chi-\frac{2}{r}\right\|_{L_{x}^{2} L_{t}^{\infty}}+\mathcal{I}_{0}+\mathcal{R}_{0}+\Delta_{0}^{2} \\
& \lesssim \mathcal{I}_{0}+\mathcal{R}_{0}+\Delta_{0}^{2}
\end{aligned}
$$

as desired. The corresponding estimates ${ }^{35}$ for $\underline{\hat{\chi}}$ can be derived in the same manner from its transport equation,

$$
\nabla_{L} \underline{\hat{\chi}}+\frac{1}{2} \operatorname{tr} \chi \cdot \underline{\hat{\chi}}=-\nabla \widehat{\otimes} \zeta-\frac{1}{2} \operatorname{tr} \underline{\chi} \cdot \hat{\chi}+\zeta \widehat{\otimes} \zeta .
$$

We also observe, taking into account the estimates we have already derived for $\operatorname{tr} \chi, \hat{\chi}, \zeta, \operatorname{tr} \underline{\chi}, \underline{\hat{x}}$

$$
\begin{aligned}
\left\|\nabla_{L} \underline{\hat{\chi}}\right\|_{L_{t}^{2} L_{x}^{2}} & \lesssim\|\operatorname{tr} \hat{\chi} \cdot \underline{\hat{\chi}}\|_{L_{t}^{2} L_{x}^{2}}+\|\nabla \zeta\|_{L_{t}^{2} L_{x}^{2}}+\|\operatorname{tr} \underline{\underline{\chi}} \cdot \hat{\chi}\|_{L_{t}^{2} L_{x}^{2}}+\|A\|_{L_{t}^{4} L_{x}^{4}}^{2} \\
& \lesssim \mathcal{I}_{0}+\mathcal{R}_{0}+\Delta_{0}^{2}+\|\operatorname{tr} \underline{\chi}\|_{L_{x}^{2} L_{t}^{\infty}}\|\hat{\chi}\|_{L_{x}^{\infty} L_{t}^{2}} \lesssim \mathcal{I}_{0}+\mathcal{R}_{0}+\Delta_{0}^{2}
\end{aligned}
$$

Similarily, from (280),

$$
\left\|\nabla_{L}\left(\operatorname{tr} \underline{\chi}+\frac{2}{r}\right)\right\|_{L_{t}^{2} L_{x}^{2}} \lesssim \mathcal{I}_{0}+\mathcal{R}_{0}+\Delta_{0}^{2} .
$$

We gather the results obtained above in the following:

\footnotetext{
${ }^{35}$ Alternatively one can use the Codazzi equations for $\underline{\hat{\chi}}$.
} 
Proposition 7.8. Assuming the boot-strap assumptions BA1-BA4 as well as the auxilliary assumption BA5 we can derive the following estimates for tr $\underline{\chi}, \underline{\hat{\chi}}$ :

$$
\begin{aligned}
\left\|t \underline{\underline{\chi}}+\frac{2}{r}\right\|_{\mathcal{B}^{0}} & \lesssim \mathcal{I}_{0}+\mathcal{R}_{0}+\Delta_{0}^{2} \\
\|\underline{\hat{\chi}}\|_{\mathcal{B}^{0}} & \lesssim \mathcal{I}_{0}+\mathcal{R}_{0}+\Delta_{0}^{2} \\
\left\|t r \underline{\chi}+\frac{2}{r}\right\|_{L_{x}^{2} L_{t}^{\infty}}+\|\underline{\hat{\chi}}\|_{L_{x}^{2} L_{t}^{\infty}} & \lesssim \mathcal{I}_{0}+\mathcal{R}_{0}+\Delta_{0}^{2} \\
\left\|\nabla_{L}\left(t r \underline{\chi}+\frac{2}{r}\right)\right\|_{L_{t}^{2} L_{x}^{2}} & \lesssim \mathcal{I}_{0}+\mathcal{R}_{0}+\Delta_{0}^{2} \\
\left\|\nabla_{L} \underline{\hat{\chi}}\right\|_{L_{t}^{2} L_{x}^{2}} & \lesssim \mathcal{I}_{0}+\mathcal{R}_{0}+\Delta_{0}^{2} .
\end{aligned}
$$

In particular we can choose $\mathcal{I}_{0}, \mathcal{R}_{0}$ sufficiently small and check that the bootstrap assumptions BA3, BA4 for tr $\underline{\chi}$ and $\hat{\chi}$ are verified with $\Delta_{0}$ replaced by $\Delta_{0} / 2$. We also deduce all the estimates for tr $\underline{\underline{\chi}}, \underline{\hat{\chi}}$ in theorem 3.6.

It only remains to verify the auxilliary assumption BA5. To achieve that we observe that, in view of (281) and (283) the terms we have denoted by $\underline{A}=\left(\operatorname{tr} \underline{\chi}+\frac{2}{r}, \underline{\hat{\chi}}\right)$ have the following structure

$$
\begin{aligned}
\nabla_{L} \underline{A}+\frac{1}{2} \operatorname{tr} \chi \underline{A} & =-\nabla_{L} P+E \\
\mathcal{N}_{1}(P),\|E\|_{\mathcal{P}^{0}} & \lesssim \mathcal{I}_{0}+\mathcal{R}_{0}+\Delta_{0}^{2}
\end{aligned}
$$

To estimate $\|A \cdot \underline{A}\|_{\mathcal{P}^{0}}$ we make use of proposition 5.9 as follows: Observe that the tensor $W=P+\underline{A}$ verifies the transport equation,

$$
\nabla_{L} W+\frac{1}{2} \operatorname{tr} \chi W=\frac{1}{2} \operatorname{tr} \chi P+E .
$$

Clearly $\|\operatorname{tr} \chi P\|_{\mathcal{P}^{0}} \lesssim \mathcal{I}_{0}+\mathcal{R}_{0}+\Delta_{0}^{2}$ and thus the term $\frac{1}{2} \operatorname{tr} \chi P$ can be incorporated in $E$. According to proposition 5.7 we have

$$
\begin{aligned}
\|A \cdot W\|_{\mathcal{P}^{0}} & \lesssim\left(\mathcal{N}_{1}(A)+\|A\|_{L_{x}^{\infty} L_{t}^{2}}\right) \cdot\left(\|W(0)\|_{B_{2,1}^{0}\left(S_{0}\right)}+\|E\|_{\mathcal{P}^{0}}\right) \\
& \lesssim \Delta_{0} \cdot\left(\mathcal{I}_{0}+\mathcal{R}_{0}+\Delta_{0}^{2}\right)
\end{aligned}
$$

Therefore,

$$
\begin{aligned}
\|A \cdot \underline{A}\|_{\mathcal{P}^{0}} & \lesssim\|A \cdot W\|_{\mathcal{P}^{0}}+\|A \cdot P\|_{\mathcal{P}^{0}} \\
& \lesssim \Delta_{0} \cdot\left(\mathcal{I}_{0}+\mathcal{R}_{0}+\Delta_{0}^{2}\right)+\mathcal{N}_{1}(G) \cdot \mathcal{N}_{1}(P) \\
& \lesssim \Delta_{0} \cdot\left(\mathcal{I}_{0}+\mathcal{R}_{0}+\Delta_{0}^{2}\right) \leq \frac{1}{2} \Delta_{0}^{2}
\end{aligned}
$$

provided that $\mathcal{I}_{0}, \mathcal{R}_{0}$ are sufficiently small relative to $\Delta_{0}$. This justifies the auxilliary bootstrap assumption BA5 and shows that the results of propositions 7.6 and 7.8 are valid without that additional assumption.

Together the propositions 7.2, 7.6 and 7.8 finish the proof of our main theorem. 


\section{Appendix}

The purpose of this appendix is to prove the commutator lemma 4.15. This requires a quick recall of some of the results of [Kl-Rodn3] for surfaces. We note that all our surfaces $S=S_{s}$, defined by the geodesic foliation on $\mathcal{H}$, verify the condition WS of proposition 4.4. In particular they satisfy the weak regularity condition of [Kl-Rodn3].

Definition 8.1. We define the negative fractional powers $\Lambda^{-a}=(I-\Delta)^{-a / 2}$, $a>0$, according to the formula,

$$
\Lambda^{-a} f=c_{a} \int_{0}^{\infty} \tau^{\frac{a}{2}-1} e^{-\tau} U(\tau) f d \tau
$$

where the heat flow $U(\tau) f=e^{\tau \Delta} f$ is defined by,

$$
\partial_{\tau} U(\tau) f-\Delta U(\tau) f=0, U(0) f=f .
$$

and $c_{a}$ appropriate constants. In fact the constnts are such that the standard composition formulas hold:

$$
\Lambda^{-a} \cdot \Lambda^{-b}=\Lambda^{-(a+b)}
$$

which reflects the semigroup properties of $U(\tau)$.

In the following proposition we record the main resullts from [Kl-Rodn3] which we shall need in the proof of lemma 4.15 .

Proposition 8.2. Given a surface $S$ verifying $\mathbf{W S}$, with $\frac{1}{2} \leq r \leq 2$, we have the following inequalities:

i) The following calculus inequalities hold for an arbitrary tensor $F$ and any $2 \leq p<\infty$,

$$
\|F\|_{L^{p}(S)} \lesssim\|\nabla F\|_{L^{2}(S)}^{1-\frac{2}{p}}\|F\|_{L^{2}(S)}^{\frac{2}{p}}+\|F\|_{L^{2}(S)}
$$

ii) The following Böchner-type inequality holds for any scalar function $f$,

$$
\left\|\nabla^{2} f\right\|_{L^{2}(S)} \lesssim\|\Delta f\|_{L^{2}(S)}+I_{a} \cdot\|\nabla f\|_{L^{2}(S)}
$$

where

$$
I_{a}=1+K_{a}^{\frac{1}{1-a}}+K_{a}^{\frac{1}{2}}, \quad K_{a}=\left\|\Lambda^{-a}\left(K-\frac{1}{r^{2}}\right)\right\|_{L^{2}(S)}
$$

iii) The heat flow $U(\tau) f=e^{\tau \Delta} f$ defined by (292) verifies the following properties:

$$
\begin{aligned}
\|U(\tau) F\|_{L^{2}(S)} & \lesssim\|F\|_{L^{2}(S)} \\
\|\nabla U(\tau) F\|_{L^{2}(S)} & \lesssim \tau^{-\frac{1}{2}}\|F\|_{L^{2}(S)} \\
\|\Delta U(\tau) F\|_{L^{2}(S)} & \lesssim \tau^{-1}\|F\|_{L^{2}(S)} \\
\|U(\tau) \nabla F\|_{L^{2}(S)} & \lesssim \tau^{-\frac{1}{2}}\|F\|_{L^{2}(S)}
\end{aligned}
$$

Also, for $2 \leq p<\infty$,

$$
\|U(\tau) f\|_{L^{p}(S)} \lesssim\left(1+\tau^{-(1-2 / p)}\right)\|f\|_{L^{2}(S)}
$$


and the dual estimate, for $1<q \leq 2$,

$$
\|U(\tau) f\|_{L^{2}(S)} \lesssim\left(1+\tau^{(1-2 / q)}\right)\|f\|_{L^{q}(S)}
$$

Proof : See part I of [Kl-Rodn3].

Proof of lemma 4.15 We have to prove the following estimate,

$$
\left\|\left[\Lambda^{-a}, \nabla_{L}\right] f\right\|_{L_{t}^{1} L_{x}^{2}} \lesssim I_{a}^{\epsilon} \cdot\|f\|_{L_{t}^{2} L_{x}^{2}}
$$

with

$$
I_{a}=1+K_{a}^{\frac{1}{1-a}}+K_{a}^{\frac{1}{2}}, \quad K_{a}=\left\|\Lambda^{-a}\left(K-\frac{1}{r^{2}}\right)\right\|_{L^{2}(S)} .
$$

Acoording to (291) and Duhamel's formula,

$$
\begin{aligned}
{\left[\Lambda^{-a}, \nabla_{L}\right] f } & =c_{a} \int_{0}^{\infty} \tau^{\frac{a}{2}-1} e^{-\tau}\left[U(\tau), \nabla_{L}\right] f d \tau \\
& =c_{a} \int_{0}^{\infty} d \tau \tau^{\frac{a}{2}-1} e^{-\tau} \int_{0}^{\tau} U\left(\tau-\tau^{\prime}\right)\left[\Delta, \nabla_{L}\right] U\left(\tau^{\prime}\right) f d \tau^{\prime}
\end{aligned}
$$

We now use the commutator formula (51) which we write in the symbolic form,

$$
\begin{aligned}
{\left[\nabla_{L}, \Delta\right] U\left(\tau^{\prime}\right) f } & =\nabla\left(\left(\frac{1}{r}+A\right) \cdot \nabla U\left(\tau^{\prime}\right) f\right)+(\nabla A+A \cdot A) \cdot \nabla U\left(\tau^{\prime}\right) f \\
& =\nabla \phi_{1}\left(\tau^{\prime}\right)+\phi_{2}\left(\tau^{\prime}\right)
\end{aligned}
$$

Thus,

$$
\begin{aligned}
{\left[\Lambda^{-a}, \nabla_{L}\right] f } & =\Phi_{1}+\Phi_{2} \\
\Phi_{1} & =c_{a} \int_{0}^{\infty} d \tau \tau^{\frac{a}{2}-1} e^{-\tau} \int_{0}^{\tau} U\left(\tau-\tau^{\prime}\right) \nabla \phi_{1}\left(\tau^{\prime}\right) d \tau^{\prime} \\
\Phi_{2} & =c_{a} \int_{0}^{\infty} d \tau \tau^{\frac{a}{2}-1} e^{-\tau} \int_{0}^{\tau} U\left(\tau-\tau^{\prime}\right) \phi_{2}\left(\tau^{\prime}\right) d \tau^{\prime}
\end{aligned}
$$

Using the heat flow estimates of proposition 8.2,

$$
\begin{aligned}
\left\|\Phi_{1}\right\|_{L_{t}^{1} L_{x}^{2}} & \lesssim \int_{0}^{\infty} d \tau \tau^{\frac{a}{2}-1} e^{-\tau} \int_{0}^{\tau}\left\|U\left(\tau-\tau^{\prime}\right) \nabla \phi_{1}\left(\tau^{\prime}\right)\right\|_{L_{t}^{1} L_{x}^{2}} d \tau^{\prime} \\
& \lesssim \int_{0}^{\infty} d \tau \tau^{\frac{a}{2}-1} e^{-\tau} \int_{0}^{\tau}\left|\tau-\tau^{\prime}\right|^{-\frac{1}{2}}\left\|\phi_{1}\left(\tau^{\prime}\right)\right\|_{L_{t}^{1} L_{x}^{2}} d \tau^{\prime}
\end{aligned}
$$

Now, using first Hölder for some $p>2$, sufficiently close to 2 , followed by the calculus, Bochner inequalities, and heat flow estimates of proposition 8.2 as well as assumption BA1, we derive,

$$
\begin{aligned}
\left\|\phi_{1}\left(\tau^{\prime}\right)\right\|_{L_{t}^{1} L_{x}^{2}} & \lesssim\|A\|_{L_{t}^{2} L_{x}^{\frac{2 p}{p-2}}} \cdot\left\|\nabla U\left(\tau^{\prime}\right) f\right\|_{L_{t}^{2} L_{x}^{p}}+\left\|r^{-1}\right\|_{L_{t}^{2} L_{x}^{\infty}}\|\nabla U(\tau)\|_{L_{t}^{2} L_{x}^{2}} \\
& \lesssim \Delta_{0}\left\|\nabla^{2} U\left(\tau^{\prime}\right) f\right\|_{L_{t}^{2} L_{x}^{2}}^{1-\frac{2}{p}} \cdot\left\|\nabla U\left(\tau^{\prime}\right) f\right\|_{L_{t}^{2} L_{x}^{2}}^{\frac{2}{p}}+\|\nabla U(\tau)\|_{L_{t}^{2} L_{x}^{2}} \\
& \lesssim \Delta_{0}\left\|\Delta U\left(\tau^{\prime}\right) f\right\|_{L_{t}^{2} L_{x}^{2}}^{1-\frac{2}{2}} \cdot\left\|\nabla U\left(\tau^{\prime}\right) f\right\|_{L_{t}^{2} L_{x}^{2}}^{\frac{2}{p}}+\left(1+\Delta_{0} I_{a}^{1-\frac{2}{p}}\right)\left\|\nabla U\left(\tau^{\prime}\right) f\right\|_{L_{t}^{2} L_{x}^{2}} \\
& \lesssim\|f\|_{L_{t}^{2} L_{x}^{2}}\left(\Delta_{0} \tau^{\prime-1+\frac{1}{p}}+\left(1+\Delta_{0} I_{a}^{1-\frac{2}{p}}\right) \cdot \tau^{\prime-\frac{1}{2}}\right)
\end{aligned}
$$


Therefore,

$$
\begin{aligned}
\left\|\Phi_{1}\right\|_{L_{t}^{1} L_{x}^{2}} & \lesssim\|f\|_{L_{t}^{2} L_{x}^{2}} \int_{0}^{\infty} \tau^{-\frac{3}{4}} e^{-\tau} \int_{0}^{\tau}\left(\tau-\tau^{\prime}\right)^{-\frac{1}{2}}\left(\Delta_{0} \tau^{\prime-1+\frac{1}{p}}+\left(1+\Delta_{0} I_{a}^{1-\frac{2}{p}}\right) \cdot \tau^{\prime-\frac{1}{2}}\right) \\
& \lesssim\left(1+\Delta_{0} I_{a}^{1-\frac{2}{p}}\right)\|f\|_{L_{t}^{2} L_{x}^{2}}
\end{aligned}
$$

or, with $p=\frac{2}{1-\epsilon}$ for some small $\epsilon>0$,

$$
\left\|\Phi_{1}\right\|_{L_{t}^{1} L_{x}^{2}} \lesssim\left(1+\Delta_{0} I_{a}^{\epsilon}\right)\|f\|_{L_{t}^{2} L_{x}^{2}}
$$

Similarly, with the help of (301), for some $1<r$ close to 1 ,

$$
\begin{aligned}
\left\|\Phi_{2}\right\|_{L_{t}^{1} L_{x}^{2}} & \lesssim \int_{0}^{\infty} d \tau \tau^{\frac{a}{2}-1} e^{-\tau} \int_{0}^{\tau}\left\|U\left(\tau-\tau^{\prime}\right) \phi_{2}\left(\tau^{\prime}\right)\right\|_{L_{t}^{1} L_{x}^{2}} d \tau^{\prime} \\
& \lesssim \int_{0}^{\infty} d \tau \tau^{\frac{a}{2}-1} e^{-\tau} \int_{0}^{\tau}\left(1+\left|\tau-\tau^{\prime}\right|\right)^{-\frac{1}{r}+\frac{1}{2}}\left\|\phi_{2}\left(\tau^{\prime}\right)\right\|_{L_{t}^{1} L_{x}^{r}} d \tau^{\prime}
\end{aligned}
$$

On the other hand, observing that $p=\frac{2 r}{2-r}>2$ can be made arbitrarily close to to if $r>1$ is close to 1 , we can estimate $\phi_{2}\left(\tau^{\prime}\right)$ precisely as $\phi_{1}\left(\tau^{\prime}\right)$,

$$
\begin{aligned}
\left\|\phi_{2}\left(\tau^{\prime}\right)\right\|_{L_{t}^{1} L_{x}^{r}} & \lesssim\|\nabla A+A \cdot A\|_{L_{t}^{2} L_{x}^{2}} \cdot\left\|\nabla U\left(\tau^{\prime}\right) f\right\|_{L_{t}^{2} L_{x}^{\frac{2 r}{2-r}}} \\
& \lesssim \Delta_{0} \cdot\left\|\nabla U\left(\tau^{\prime}\right) f\right\|_{L_{t}^{2} L_{x}^{p}} \\
& \lesssim \Delta_{0} \cdot\|f\|_{L_{t}^{2} L_{x}^{2}}\left(\tau^{\prime-1+\frac{1}{p}}+I_{a}^{1-\frac{2}{p}} \cdot{\tau^{\prime}}^{-\frac{1}{2}}\right), \quad p=\frac{2 r}{2-r}
\end{aligned}
$$

Thus, just as for $\Phi_{1}$, with $r=\frac{1}{1-\epsilon}$,

$$
\left\|\Phi_{1}\right\|_{L_{t}^{1} L_{x}^{2}} \lesssim \Delta_{0} I_{a}^{\epsilon}\|f\|_{L_{t}^{2} L_{x}^{2}}
$$

Combining (303) with (304) we deduce,

$$
\left\|\left[\Lambda^{-a}, \nabla_{L}\right] f\right\|_{L_{t}^{1} L_{x}^{2}} \lesssim\left(1+\Delta_{0} I_{a}^{\epsilon}\right)\|f\|_{L_{t}^{2} L_{x}^{2}} \lesssim I_{a}^{\epsilon}\|f\|_{L_{t}^{2} L_{x}^{2}}
$$

as desired.

\section{REFERENCES}

[Ba-Ch1] H. Bahouri and J. Y. Chemin. Équations d'ondes quasilinéaires et estimation de Strichartz. Amer. J. Math., vol. 121; (1999), pp. 1337-1777

[Ba-Ch2] H. Bahouri and J. Y. Chemin. Équations d'ondes quasilinéaires et effet dispersif. IMRN, vol. 21; (1999), pp. 1141-1178

[B] J.M. Bony Calcul Symbolique et propagation des singularité pour les equations aux dérivées partielles nonlinéares, Ann. Sci. École Norm. Sup 14, 209-256.

[Br] Y. Choquet Bruhat Theoreme d'Existence pour certains systemes d'equations aux derivees partielles nonlineaires., Acta Math. 88 (1952), 141-225.

[Chr-Kl] D.Christodoulou and S. Klainerman. The Global Nonlinear Stability of the Minkowski Space. Princeton Mathematical Series, 41. Princeton University Press, 1993

[H-K-M] Hughes, T. Kato and J. Marsden Well posed quasilinear second order hyperbolic systems Arch. Rat. Mech. Anal. 63(1976) no 3, 273-294.

[F-S] H. Friedrich and J.M. Stewart Characteristic initial data and wave front singulariries in general relativity Proc. Roy.Soc. Lond. A385, 345-371.

[Kl1] S. Klainerman. PDE as a unified subject Special Volume GAFA 2000, 279-315

[K12] S. Klainerman. A commuting vectorfield approach to Strichartz type inequalities and applications to quasilinear wave equations. IMRN, 2001, No 5, 221-274.

[Kl-Ma] S. Klainerman and M. Machedon, Space-time estimates for null forms and the local existence theorem, CPAM 46 (1993), no. 9, 1221-1268 
[Kl-Nic] S. Klainerman and F. Nicolo, The Evolution problem in General Relativity, Progress in Mathematical Physics 25, Birkhauser.

[Kl-Rodn1] S. Klainerman and I. Rodnianski, Improved local well posedness for quasilinear wave equations in dimension three, Duke Math. J. 117 (2003), no. 1, 1-124

[Kl-Rodn2] S. Klainerman and I. Rodnianski, Rough solutions of the Einstein vacuum equations to appear in Annals of Math.

[Kl-Rodn3] S. Klainerman and I. Rodnianski, A geometric version of Litlewood-Paley theory. preprint

[Kl-Rodn4] S. Klainerman and I. Rodnianski, Sharp Trace Theorems on null hypersurfaces in Einstein backgrounds with finite curvature flux. preprint

[Lind] H. Linblad. Counterexamples to local existence for semilinear wave equations. AJM, vol. 118; (1996), pp. 1-16

[Ta] D. Tataru. Strichartz estimates for second order hyperbolic operators with non smooth coefficients. III, JAMS 15 (2002), no.2, 419-442 Preprint

[Sm-Ta] H. Smith and D. Tataru. Sharp local well posedness results for the nonlinear wave equation. Preprint

[Stein] E. Stein Topics in harmonic analysis related to Littlewood-Paley theory, Annals of Mathematical Studies, Princeton University Press 63(1970), 145 pages.

Department of Mathematics, Princeton University, Princeton NJ 08544

E-mail address: $\quad$ seri@math.princeton.edu

Department of Mathematics, Princeton University, Princeton NJ 08544

E-mail address: irod@math.princeton.edu 